\title{
Evasion of chitin-triggered immunity by fungal plant pathogens
}

\author{
Hanna J. Rövenich
}




\section{Propositions}

1. VdAve1 is an effector protein with dual virulence function. (this thesis)

2. Evolution of filamentous microbes toward suppression of glycan-triggered immunity is a basic requirement for establishment in any niche. (this thesis)

3. The technologies that are being developed by environmental engineers and nanoscale scientists to harvest the energy generated by plants during photosynthesis have the potential to revolutionize the energy market.

4. The next step to enhance our understanding of the mechanisms underlying host-microbe interactions will be to integrate the knowledge on hostassociated microbiomes.

5. Courses on science communication and outreach activities should be core requirements of each scientist's education.

6. Learning to speak foreign languages as adults is a matter of mindset.

Propositions belonging to the thesis, entitled

Evasion of chitin-triggered immunity by fungal plant pathogens

Hanna J. Rövenich

Wageningen, 29 August 2017 


\section{Evasion of chitin-triggered immunity by fungal plant pathogens}

Hanna J. Rövenich 


\section{Thesis committee}

\section{Promotors}

Prof. Dr B.P.H.J. Thomma

Professor of Phytopathology

Wageningen University \& Research

Prof. Dr P.J.G.M. de Wit

Professor of Phytopathology

Wageningen University \& Research

\section{Other members}

Prof. Dr J. van der Oost, Wageningen University \& Research

Prof. Dr E.H. Stukenbrock, University of Kiel, Germany

Dr A. Goverse, Wageningen University \& Research

Dr R. Geurts, Wageningen University \& Research

This research was conducted under the auspices of the Graduate School of Experimental Plant Sciences. 


\title{
Evasion of chitin-triggered immunity by fungal plant pathogens
}

\author{
Hanna J. Rövenich
}

Thesis

submitted in fulfillment of the requirements for the degree of doctor

at Wageningen University

by the authority of the Rector Magnificus,

Prof. Dr A.P.J. Mol,

in the presence of the

Thesis Committee appointed by the Academic Board

to be defended in public

on Tuesday 29 August 2017

at 11 a.m. in the Aula. 
Hanna J. Rövenich

Evasion of chitin-triggered immunity by fungal plant pathogens, 134 pages.

PhD thesis, Wageningen University, Wageningen, The Netherlands (2017) With references, with summary in English

DOI: $10.18174 / 418026$

ISBN: 978-94-6343-613-7 


\section{Contents}

Chapter 1 General introduction 7

Chapter 2 Filamentous pathogen effector functions: Of pathogens, hosts and microbiomes

Chapter 3 The fungal effector protein Ecp6 interferes with chitin perception in the model plant Arabidopsis thaliana

Chapter 4 A LysM receptor kinase mediates chitin-triggered defense responses in tomato

Chapter 5 Neofunctionalisation after horizontal gene transfer: birth of a fungal effector that evolved towards immune suppression

Chapter 6 The tomato immune receptor Ve1 binds the Verticillium dahliae effector protein VdAve1 to recruit downstream signaling components

About the author

List of publications 



\section{Chapter 1}

General introduction 


\section{Conceptual models of plant immunity}

Plants establish very intimate, symbiotic relationships with microbes. However, as in animals, microbial colonization of plant hosts only rarely results in disease. This is mainly due to the presence of a complex immune system, which allows plants to survey their environment for the presence of potential pathogens through the activity of plant immune receptors'.

The interaction between plant immune receptors and pathogen ligands was originally defined in the "gene-for-gene" hypothesis, which postulated that the products of singledominant plant resistance $(R)$ genes recognize the products of corresponding pathogen avirulence (Avr) genes to activate race-specific resistance ${ }^{2,3}$. However, this model failed to explain the function of so-called general elicitors, which defied the rules of race-specificity and were recognized by multiple plant species ${ }^{4}$. It was not until the introduction of the "zigzag" model that these seemingly different observations were combined into a single concept describing plant immunity ${ }^{5}$. In this model, the first line of defence is governed by cell surface-localized pattern recognition receptors (PRRs) that detect pathogenassociated molecules patterns (PAMPs) to activate broad-spectrum disease resistance, called PAMP-triggered immunity (PTI). As PAMPs include molecules that are present both in pathogenic as well as non-pathogenic microbes ${ }^{6}$, they have been renamed microbeassociated molecular patterns (MAMPs), whose recognition leads to the establishment of MAMP-triggered immunity (MTI). In turn, successful microbes employ effector molecules to overcome MTI resulting in effector-triggered susceptibility (ETS). In the second layer of defense, effectors are recognized by intracellular receptors (R proteins) that activate effectortriggered immunity (ETI). Microbes may evade recognition through loss or mutation of recognized effectors or actively suppress ETI using novel effectors. These processes describe the continued coevolution between microbes and their hosts, which has been termed the molecular "arms race".

In addition to successfully incorporating the observations made about general elicitors, the "zigzag" model recognizes the role of effector molecules as contributors to microbial virulence irrespective of the host genotype. However, the strict separation of MTI and ETI layers of immunity in the "zigzag"model does not account for the spatio-temporal continuum underlying plant-microbe interactions ${ }^{7,8}$. In addition, is does not resolve the conceptual conflict that MAMPs are defined from the perspective of the host, whereas effectors are defined from the perspective of the invading microbe ${ }^{8}$. These discrepancies have led to the formulation of the Invasion Model, in which host receptors (referred to as invasion pattern receptors, IPRs) recognize microbe-derived or modified-self ligands (invasion patterns, IPs) that reveal invasion ${ }^{8}$. While any molecule can serve as IP that is detected by an IPR, the probability of a particular ligand-receptor complex to evolve within the framework of host immunity increases with the probability that the ligand retains its function, conservation across organisms, importance in establishment of symbiosis, and accessibility ${ }^{8}$. 


\section{Main objective and research questions}

With the availability of high-quality genome and transcriptome data for many microbial species, the number of identified putative effector molecules has rapidly increased in recent years. However, the functions of most of these effectors, as well as the mechanisms governing their recognition by plant receptors, have largely remained unexplored. The main objective of my doctoral research was to tackle this gap specifically in plantfungal interactions. By using a combination of physiological, biochemical, and proteomic approaches I addressed the following general questions:

1. How do plants perceive microbe-derived molecules?

2. What are the roles of effector proteins during host plant infection and how do they contribute to virulence of different fungal pathogens?

\section{Study systems}

\section{The host model plant tomato}

Tomato (Solanum lycopersicum L.) is considered one of the most important vegetable crops with a worldwide distribution and a net economic value exceeding $\$ 55$ billion $^{9}$. After several decades of breeding efforts for the introduction of resistance loci into cultivated accessions, and with the increasing availability of genomic resources, tomato has become a particularly important model crop for the study of plant-pathogen interactions. In addition, it represents an important model species for biological research on the genetic improvement of Solanaceous crops, including pepper, potato and eggplant ${ }^{10}$.

\section{The broad host-range pathogen Verticillium dahliae}

The Verticillium genus consist of ten currently recognized fungal ascomycete species whose lifestyles range from saprotrophic to symbiotic ${ }^{11}$. Among the symbionts, Verticillium dahliae is the most detrimental pathogen as it is able to infect over 200 dicotyledonous plant species including many crops, such as tomato ${ }^{12}$. It has been estimated that wilt disease caused by Verticillium spp. results in billions of dollars of annual economic losses ${ }^{13,14}$.

$V$. dahliae resting structures, called microsclerotia, reside within the soil where they germinate in response to plant root exudates ${ }^{15}$. Emerging hyphae penetrate the host tissue at root tips or at sites of lateral root formation ${ }^{16}$. In susceptible plants, the fungus colonizes the vascular system after invasive hyphae have crossed the root endodermis. Conidia formation and transport throughout the vasculature eventually results in systemic colonization $^{15}$. This causes chlorosis, necrosis and wilting of the host plant. The fungus 
then enters a saprotrophic stage during which it colonizes necrotic and senescent plant tissues. At these late infection stages, $V$. dahliae produces large amounts of microsclerotia that are released into the soil upon decomposition of the host plant tissue, where they can survive for 10-15 years ${ }^{17}$. Due to the broad host range of $V$. dahliae and the long-term prevalence of its resting structures in the soil, agricultural practices such as crop rotation do not result in crop protection ${ }^{15}$. Disease control is particularly difficult as fungicides are generally ineffective once the pathogen has entered the vascular system, and soil fumigation has largely been banned due to harmful effects on the environment and/or public health. Therefore, the preferred method for disease control is genetic resistance.

Genetic resistance to $V$. dahliae has been identified in several crop species ${ }^{12,18-21}$. In tomato, the Ve locus was described to confer resistance against race 1 but not race 2 isolates of $V$. dahliae ${ }^{22,23}$, and has been introduced into most cultivated tomato genotypes ${ }^{15}$. Positional cloning showed that the Ve locus contains two closely linked genes, Ve1 and Ve2, both of which encode membrane-bound extracytoplasmic leucine-rich repeat receptor proteins (eLRR-RPs) ${ }^{24}$. While both Ve1 and Ve2 conferred resistance to pathogenic V. albo-atrum when expressed in susceptible potato plants, only Ve1 could be confirmed to provide resistance against race 1 isolates in tomato and other plant species ${ }^{21,24,25}$.

\section{Cladosporium fulvum, the tomato specialist}

In contrast to $V$. dahliae, Cladosporium fulvum is a non-obligate biotrophic fungus that causes leaf mold on tomato. On susceptible plants, $C$. fulvum conidia germinate on the abaxial side of a leaf and hyphae enter the plant through open stomata to invade leaf intercellular spaces ${ }^{26,27}$. Despite the lack of feeding structure formation, C. fulvum growth seems to rely on the physical contact with host cells in close proximity to vascular tissues. This is thought to be due to the sucrose gradient that is established around the phloem ${ }^{28,29}$. The first disease symptoms appear at approximately one week after the start of infection as pale green or yellow spots on the upper leaf surface as well as white to olive-green patches of mold on the abaxial sides of leaves. These turn brown upon sporulation at 10-14 days. In severe cases, sporulation is associated with leaf wilting and may lead to plant death ${ }^{26}$.

Like other plant pathogens, C. fulvum employs effector proteins to successfully infect susceptible tomato plants. So far, 13 effectors have been identified and the corresponding genes have been cloned $^{30-39}$. While the three C. fulvum effectors Avr2 ${ }^{40-42}$, Avr4 ${ }^{43-45}$, and Ecp $6^{30,46,47}$ have been functionally characterized, the intrinsic function of most of them remains unknown. In resistant tomato accessions, C. fulvum effectors are recognized by $C f$ resistance genes that, like $V e 1$ and $V e 2$, encode eLRR-RPs ${ }^{48}$. This triggers immune responses, which ultimately result in a hypersensitive response (HR), a form of localized cell death that halts pathogen growth at the infection site ${ }^{49}$. Most of the $C f$ genes that have been introgressed into cultivated tomatoes originate from wild Solanum species and landraces ${ }^{50}$. Even though the use of resistant cultivars has been effective in containing the 
pathogen, their intensive cultivation has led to the emergence of novel C. fulvum strains capable of overcoming cloned $C f$ receptor genes ${ }^{51-54}$.

\section{Thesis outline}

Plant hosts employ surface-localized receptor molecules to survey their environment for the presence of potentially harmful microbes. These receptors perceive ligands, which are either microbe-derived or result from microbe-mediated plant manipulation, to activate immunity. In order to circumvent recognition or suppress immune responses, microbes secrete effector proteins that deregulate host physiological processes. The emphasis of the work presented here lies within the identification and functional characterization of tomato receptor proteins involved in microbe recognition, and the effector proteins employed by the fungal pathogens C. fulvum and $V$. dahliae to facilitate tomato colonization.

Effectors are not unique to pathogens but are employed by any microbe that encounters immune responses during plant host colonization. Moreover, plant-microbe interactions occur in environments that contain additional microbial partners, which can affect the colonizing microbe as well as the host plant. Chapter 2 reviews the role of effector molecules secreted by pathogenic filamentous microbes in the suppression of plant immune responses, and proposes their involvement in microbial competition or cooperation to shape plant microbiomes.

Chitin is a major structural component of fungal cell walls. During host colonization chitin recognition results in the activation defense responses that threaten the survival of fungal invaders. The tomato leaf mold pathogen C. fulvum has evolved several strategies to prevent chitin recognition during colonization of its tomato host. The $C$. fulvum effector protein Ecp6 has previously been shown to sequester chitin fragments released from the fungal cell wall to suppress their recognition. However, Ecp6 has been hypothesized to additionally interfere with the formation of host chitin receptor complexes required for the activation of chitin-triggered immunity. Chapter $\mathbf{3}$ addresses this hypothesis using the model plant species Arabidopsis thaliana.

While chitin recognition has been intensely studied in $A$. thaliana and rice, little is known about chitin perception in tomato. In Chapter 4, we present a proteomics approach that led to the identification of putative tomato chitin receptor candidates. Subsequent oxidative burst and gene expression assays confirmed that silencing of a single candidate is sufficient to reduce chitin responsiveness in tomato.

$V$. dahliae is a successful pathogen on hundreds of plant species. Through comparative genomics the effector protein Ave1 has recently been identified as a major virulence factor during $V$. dahliae infection of susceptible tomato and other hosts. Phylogenetic analysis 
demonstrated that, in contrast to many other effectors, Ave1 has homologs in several plant pathogens as well as numerous plants. Based on this phylogenetic distribution, it has been hypothesized that Ave 1 has been acquired by $V$. dahliae through horizontal gene transfer. Chapter 5 describes the functional characterization of Ave1, revealing that this effector has functionally diverged from its microbial and plant homologs.

In tomato cultivars that carry the Ve locus, recognition of Ave1 by the leucine-rich repeatcontaining receptor protein Ve1 results in resistance to $V$. dahliae. The Ve locus contains a second gene, Ve2, to which no function could be ascribed despite its homology to Ve1. Nonetheless, both Ve1 and Ve2 were shown to bind the adaptor receptor SOBIR1 in a ligand-independent manner. In Chapter 6, we use a biochemical approach to further investigate the composition of the Ve1 receptor complex upon ligand binding and address the question of the lack of Ve2 function in V. dahliae resistance.

Chapter 7 places the most important findings of this thesis into the broader context of glycan-triggered immunity in plants, and discusses the importance of its evasion by filamentous pathogens.

\section{References}

1 Dodds, P. N. \& Rathjen, J. P. Plant immunity: towards an integrated view of plant-pathogen interactions. Nat. Rev. Genet. 11, 539-548, doi:10.1038/nrg2812 (2010).

2 Flor, H. H. Current status of the gene-for-gene concept. Annu. Rev. Phytopathol. 9, 275-296, doi:10.1146/annurev.py.09.090171.001423 (1971).

3 Flor, H. H. Inheritance of pathogenicity in Melampsora lini. Phytopathology 32, 653-669 (1942).

4 Darvill, A. G., Albersheim, P. Phytoalexins and their elicitors - a defence against microbial infection in plants. Annu. Rev. Plant Physiol. 35, 243-275, doi:10.1146/annurev.pp.35.060184.001331 (1984).

5 Jones, J. D. \& Dangl, J. L. The plant immune system. Nature 444, 323-329, doi:10.1038/nature05286 (2006).

6 Ausubel, F. M. Are innate immune signaling pathways in plants and animals conserved? Nat. Immunol. 6, 973-979, doi:10.1038/ni1253 (2005).

7 Thomma, B. P. H. J., Nürnberger, T. \& Joosten, M. H. A. J. Of PAMPs and effectors: the blurred PTIETI dichotomy. Plant Cell 23, 4-15, doi:10.1105/ tpc110.082602 (2011).
8 Cook, D. E., Mesarich, C. H. \& Thomma, B. P. H. J. Understanding plant immunity as a surveillance system to detect invasion. Annu. Rev. Phytopathol. 53, 541-563, doi:10.1146/annurevphyto-080614-120114 (2015).

9 Vincent, H., Wiersema, J., Kell, S., Fielder, H., Dobbie, S., Castañeda-Álvarez, N.P., Guarino, L., Eastwood, R., León, B., Maxted, N. A prioritized crop wild relative inventory to help underpin global food security. Biol. Conserv. 167, 265-275, doi:10.1016/j. biocon.2013.08.011 (2013).

10 Lin, T.et al. Genomic analyses provide insights into the history of tomato breeding. Nat. Genet. 46, 1220-1226, doi:10.1038/ng.3117 (2014).

11 Inderbitzin, P. et al. Phylogenetics and taxonomy of the fungal vascular wilt pathogen Verticillium, with the descriptions of five new species. PLOS ONE 6, e28341, doi:10.1371/journal.pone.0028341 (2011).

12 Pegg, G. F., Brady, B.L. Verticillium wilts (CABI Publishing, Wallingford, Oxfordshire, 2002).

13 Klosterman, S. J., Atallah, Z. K., Vallad, G. E. \& Subbarao, K. V. Diversity, pathogenicity, and management of Verticillium species. Annu. Rev. Phytopathol. 47, 39-62, doi:10.1146/annurevphyto-080508-081748 (2009). 
14 Depotter, J. R. et al. Verticillium longisporum, the invisible threat to oilseed rape and other brassicaceous plant hosts. Mol. Plant Pathol. 17, 1004-1016, doi:10.1111/mpp.12350 (2016).

15 Fradin, E. F. \& Thomma, B. P. H. J. Physiology and molecular aspects of Verticillium wilt diseases caused by V. dahliae and V. albo-atrum. Mol. Plant Pathol. 7, 71-86, doi:10.1111/J.1364-3703.2006.00323.X (2006).

16 Bishop, C. D., Cooper, R.M. An ultrastructural study of root invasion in three vascular wilt diseases. Physiol. Plant Pathol. 22, 15-27, doi:10.1016/S00484059(83)81034-0 (1983).

17 Wilhelm, S. Longevity of the Verticillium wilt fungus in the laboratory and in the field. Phytopathol. $\mathbf{4 5}$, 180-181 (1955).

18 Lynch, D. R., Kawchuk, L.M., Hachey J., Bains, P.S., Howard, R.J. Identification of a gene conferring high levels of resistance to Verticillium wilt in Solanum chacoense. Plant Dis. 81, 1011-1014, doi:10.1094/ PDIS.1997.81.9.1011 (1997).

19 Hayes, R. J., Vallad, G.E., Qin, Q.-M., Grube R.C., Subbarao, K.V. Variation in resistance to Verticillium wilt in lettuce (Latuca sativa L.). Plant Dis. 91, 439445, doi:10.1094/PDIS-91-4-0439 (2007).

20 Rygulla, W. et al. Identification of quantitative trait loci for resistance against Verticillium longisporum in oilseed rape (Brassica napus). Phytopathol. 98, 215221, doi:10.1094/PHYTO-98-2-0215 (2008).

21 Song, Y. et al. Broad taxonomic characterization of Verticillium wilt resistance genes reveals an ancient origin of the tomato Ve1 immune receptor. Mol. Plant Pathol. 18, 195-209, doi:10.1111/mpp.12390 (2017).

22 Schaible, L., Cannon, O.S., Waddoups, V. Inheritance of resistance to Verticillium wilt in a tomato cross. Phytopathol. 41, 986-990 (1951).

23 Pegg, G. F. Verticillium diseases. Rev. Plant Pathol. 53, 157-182 (1974).

24 Kawchuk, L. M. et al. Tomato Ve disease resistance genes encode cell surface-like receptors. Proc. Natl. Acad. Sci. USA 98, 6511-6515, doi:10.1073/ pnas.091114198 (2001).

25 Fradin, E. F.et al. Genetic dissection of Verticillium wilt resistance mediated by tomato Ve1. Plant Physiol. 150, 320-332, doi:10.1104/pp.109.136762 (2009).

26 Thomma, B. P. H. J., van Esse, H. P., Crous, P. W. \& de Wit, P. J. G. M. Cladosporium fulvum (syn. Passalora fulva), a highly specialized plant pathogen as a model for functional studies on plant pathogenic Mycosphaerellaceae. Mol. Plant Pathol. 6, 379-393, doi:10.1111/j.1364-3703.2005.00292.x (2005).
27 Rivas, S. \& Thomas, C. M. Molecular interactions between tomato and the leaf mold pathogen Cladosporium fulvum. Annu. Rev. Phytopathol. 43, 395-436, doi:10.1146/annurev. phyto.43.040204.140224 (2005).

28 van den Ackerveken, G. F. et al. Nitrogen limitation induces expression of the avirulence gene Avr9 in the tomato pathogen Cladosporium fulvum. Mol. Gen. Genet. 243, 277-285 (1994).

29 Wubben, J. P., Joosten, M. H. A. J. \& De Wit, P. J. G. M. Expression and localization of two in planta induced extracellular proteins of the fungal tomato pathogen Cladosporium fulvum. Mol. Plant-Microbe Interact. 7, 516-524 (1994).

30 Bolton, M. D. et al. The novel Cladosporium fulvum lysin motif effector Ecp6 is a virulence factor with orthologues in other fungal species. Mol. Microbiol. 69, 119-136, doi:10.1111/j.13652958.2008.06270.x (2008).

31 Joosten, M. H. A. J., Cozijnsen, T. J. \& de Wit, P. J. G. $M$. Host resistance to a fungal tomato pathogen lost by a single base-pair change in an avirulence gene. Nature 367, 384-386, doi:10.1038/367384a0 (1994).

32 Laugé, R., Goodwin, P.H., deWit, P.J.G.M. \& Joosten, M. H. A. J. Specific HR-associated recognition of secreted proteins from Cladosporium fulvum occurs in both host and non-host plants. Plant J. 23, 735-745 (2000).

33 Luderer, R., Takken, F. L., de Wit, P. J. G. M. \& Joosten, M. H. A. J. Cladosporium fulvum overcomes Cf2-mediated resistance by producing truncated AVR2 elicitor proteins. Mol. Microbiol. 45, 875-884 (2002).

34 Mesarich, C. H. et al. Transcriptome sequencing uncovers the Avr5 avirulence gene of the tomato leaf mold pathogen Cladosporium fulvum. Mol. Plant-Microbe Interact. 27, 846-857, doi:10.1094/ MPMI-02-14-0050-R (2014).

35 Ökmen, B. et al. Detoxification of alpha-tomatine by Cladosporium fulvum is required for full virulence on tomato. New Phytol. 198, 1203-1214, doi:10.1111/nph.12208 (2013).

36 Stergiopoulos, I. et al. In silico characterization and molecular evolutionary analysis of a novel superfamily of fungal effector proteins. Mol. Biol. Evol. 29, 3371-3384, doi:10.1093/molbev/mss143 (2012). 
37 van den Ackerveken, G. F. et al. Characterization of two putative pathogenicity genes of the fungal tomato pathogen Cladosporium fulvum. Mol. PlantMicrobe Interact. 6, 210-215 (1993).

38 van Kan, J. A., van den Ackerveken, G. F. \& de Wit, P. J. G. M. Cloning and characterization of cDNA of avirulence gene Avr9 of the fungal pathogen Cladosporium fulvum, causal agent of tomato leaf mold. Mol. Plant-Microbe Interact. 4, 52-59 (1991).

39 Westerink, N., Brandwagt, B. F., de Wit, P. J. G. M. \& Joosten, M. H. A. J. Cladosporium fulvum circumvents the second functional resistance gene homologue at the Cf-4 locus (Hcr9-4E) by secretion of a stable Avr4E isoform. Mol. Microbiol. 54, 533545, doi:10.1111/j.1365-2958.2004.04288.x (2004).

40 Rooney, H. C. et al. Cladosporium Avr2 inhibits tomato $\mathrm{Rcr} 3$ protease required for $\mathrm{Cf}$-2-dependent disease resistance. Science 308, 1783-1786, doi:10.1126/science.1111404 (2005).

41 Shabab, M. et al. Fungal effector protein AVR2 targets diversifying defense-related cys proteases of tomato. Plant Cell 20, 1169-1183, doi:10.1105/ tpc.107.056325 (2008).

42 van Esse, H. P. et al. The Cladosporium fulvum virulence protein Avr2 inhibits host proteases required for basal defense. Plant Cell 20, 19481963, doi:10.1105/tpc.108.059394 (2008).

43 van den Burg, H. A., Harrison, S. J., Joosten, M. H. A. J., Vervoort, J. \& de Wit, P. J. G. M. Cladosporium fulvum Avr4 protects fungal cell walls against hydrolysis by plant chitinases accumulating during infection. Mol. Plant-Microbe Interact. 19, 1420-1430, doi:10.1094/MPMI-19-1420 (2006).

44 van den Burg, H. A. et al. Binding of the AVR4 elicitor of Cladosporium fulvum to chitotriose units is facilitated by positive allosteric protein-protein interactions: the chitin-binding site of AVR4 represents a novel binding site on the folding scaffold shared between the invertebrate and the plant chitin-binding domain. J. Biol. Chem. 279, 16786-16796, doi:10.1074/jbc.M312594200 (2004).
45 van Esse, H. P., Bolton, M. D., Stergiopoulos, I., de Wit, P. J. G. M. \& Thomma, B. P. H. J. The chitinbinding Cladosporium fulvum effector protein Avr4 is a virulence factor. Mol. Plant-Microbe Interact. 20, 1092-1101, doi:10.1094/MPMI-20-91092 (2007).

46 de Jonge, R. et al. Conserved fungal LysM effector Ecp6 prevents chitin-triggered immunity in plants. Science 329, 953-955, doi:10.1126/ science.1190859 (2010).

47 Sánchez-Vallet, A. et al. Fungal effector Ecp6 outcompetes host immune receptor for chitin binding through intrachain LysM dimerization. Elife, doi:10.7554/eLife.00790 (2013).

48 de Wit, P. J. G. M., Joosten M. H. A. J., Thomma, B. P. H. J., Stergiopoulos, I. in Plant Relationships (ed H. B. Deising) 135-156 (Springer, Berlin-Heidelberg, 2009).

49 Heath, M. C. Hypersensitive response-related death. Plant Mol. Biol. 44, 321-334 (2000).

50 Kerr, E. A., Bailey, D.L. Resistance to Cladosporium fulvum Cke. obtained from wild species of tomato. Can. J. Bot. 42, 1541-1554, doi:10.1139/b64-153 (1964).

51 Hubbeling, N. Breakdown of resistance of the Cf5 gene in tomato by another new race of Fulvia fulva. Meded. Fac. Landbouwwet. Rijksuniv. Gent 43, 891-894 (1978).

52 lida, Y.et al. Novel mutations detected in avirulence genes overcoming tomato $C f$ resistance genes in isolates of a Japanese population of Cladosporium fulvum. PLOS ONE 10, e0123271, doi:10.1371/ journal.pone.0123271 (2015).

53 Laterrot, H. Race 2.5.9, a new race of Cladosporium fulvum (Fulvia fulva) and sources of resistance in tomato. Neth. J. PLant Pathol. 92, 305-307 (1986).

54 Li, S., Zhao, T., Li, H., Xu, X., Li, J. First report of races 2.5 and 2.4 .5 of Cladosporium fulvum (syn. Passalora fulva), causal fungus of tomato leaf mold disease in China. J. Gen. Plant. Pathol. 81, 162-165, doi:10.1007/s10327-015-0577-z (2015). 


\section{Chapter 2}

Filamentous pathogen effector functions: of pathogens, hosts and microbiomes

Hanna Rovenich, Jordi C. Boshoven, Bart P.H.J. Thomma Laboratory of Phytopathology, Wageningen University and Research, Wageningen, The Netherlands 


\begin{abstract}
Microorganisms play essential roles in almost every environment on earth. For instance, microbes decompose organic material, or establish symbiotic relationships that range from pathogenic to mutualistic. Symbiotic relationships have been particularly well studied for microbial plant pathogens and have emphasized the role of effectors; secreted molecules that support host colonization. Most effectors characterized thus far play roles in deregulation of host immunity. Arguably, however, pathogens not only deal with immune responses during host colonization, but also encounter other microbes including competitors, (myco)parasites and even potential co-operators. Thus, part of the effector catalog may target microbiome co-inhabitants rather than host physiology.
\end{abstract}




\section{Introduction}

During early microbial colonization stages, plant cell surface-localized pattern recognition receptors (PRRs) recognize microbe-associated molecular patterns (MAMPs), such as fungal chitin, to activate immune responses ${ }^{1,2}$. In order to establish themselves, adapted pathogens secrete effector molecules that deregulate immune responses and facilitate host colonization. Simultaneously, hosts evolve effector recognition by novel receptors that reinstall immunity ${ }^{1,2}$. Consequently, effectors are subject to various selective forces that drive their evolution, leading to diversified effector repertoires between pathogen lineages. Functional characterization of effectors and determination of their contribution to the microbial lifestyle provides insight in relevant processes for host colonization.

\section{Plant pathogen effectors deregulate host immunity in various subcellular compartments}

Many pathogens initially enter the plant apoplast, which contains enzymes that hamper microbial colonization. For example, chitinases target fungal cell walls to release chitin fragments that activate immune receptors, leading to further chitinase accumulation to induce hyphal lysis. In turn, fungal pathogens secrete chitin-binding effectors to protect their cell walls and interfere with immune receptor activation ${ }^{3-6}$. The LysM domaincontaining Ecp6 effector of the leaf mold fungus Cladosporium fulvum can outcompete host receptors through chitin binding with unprecedented ultrahigh (pM) affinity by intramolecular LysM domain dimerization?. Additionally, LysM effectors likely interfere with receptor dimerization that is required to activate immune signaling ${ }^{7-9}$.

Although effectors that directly target chitinases have not yet been identified, some effectors target other apoplastic hydrolytic enzymes, such as proteases. For example, sequence-unrelated effectors of $C$. fulvum, the oomycete Phytophthora infestans, and the parasitic nematode Globodera rostochiensis inhibit tomato cysteine proteases including Rcr $3^{10-12}$. The closely related oomycetes $P$. infestans and $P$. mirabilis express an orthologous pair of host protease inhibitor effectors that are subject to positive selection, which was implicated in adaptation to unique protease targets in their respective host plants ${ }^{13}$. Besides protease inhibitors, $P$. infestans secretes the Avrblb2 effector that interferes with protease secretion ${ }^{14}$. The smut fungus Ustilago maydis inhibits apoplastic proteases via multiple effectors. While Pit2 directly inhibits cysteine proteases ${ }^{15}$, Pep1 induces the maize cystatin CC9 that inhibits apoplastic proteases in turn ${ }^{16}$. Pep1 furthermore inhibits the maize peroxidase POX12 to perturb reactive oxygen species balances ${ }^{17}$. Thus, the plant apoplast is a dynamic battlefield for plant pathogens. 
In addition to apoplastic effectors, many pathogens deliver effectors that act inside host cells, although mechanisms that govern their uptake remain controversial ${ }^{18}$. The rice blast fungus Magnaporthe oryzae was shown to secrete various effectors that enter rice cells, and even move to non-infected neighboring cells, presumably to prepare these for infection ${ }^{19}$. The AvrPiz-t effector targets proteasome activity through interaction with the RING E3 ubiquitin ligase APIP6, leading to their mutual degradation and suppression of PRR-mediated immunity ${ }^{20}$. Effector diffusion from infected cells into neighboring cells was similarly observed for the $U$. maydis chorismate mutase $\mathrm{Cmu} 1$ that targets the shikimate pathway to channel chorismate into the phenylpropanoid pathway, thus adversely affecting salicylic acid (SA) biosynthesis ${ }^{21}$. U. maydis furthermore secretes the Tin2 effector to stabilize the maize ZmTTK1 kinase that controls anthocyanin biosynthesis, possibly to suppress tissue lignification ${ }^{22}$. Also the oomycete Hyaloperonospora arabidopsidis targets SA signaling by secreting a nuclear-localized effector that interacts with the mediator complex that controls interactions between transcriptional regulators and RNA polymerase ${ }^{23}$. Host transcription is furthermore perturbed by effectors that inhibit transcription factor translocation to the nucleus ${ }^{24}$. Additionally, nuclear-localized effectors may affect host immunity post-transcriptionally by suppressing the biogenesis of small RNAs in the host ${ }^{25}$. Interestingly, Botrytis cinerea was recently suggested to deliver even small RNAs into host cells to affect immune responses ${ }^{26}$.

Finally, several effectors target host cell death mechanisms, such as P. infestans Avr3a and PexRD2. While Avr3a suppresses INF1-triggered cell death by stabilizing the U-box E3 ligase CMPG1 during biotrophic growth, PexRD2 targets the kinase domain of the cell death regulator MAPKKK $\varepsilon^{27,28}$. During later stages of infection, however, P. infestans relies on induction of host cell death as it switches to a necrotrophic lifestyle. Necrotrophic pathogens evolved effectors that actually induce cell death. An elegant example is provided by the Cochliobolus victoriae effector victorin that binds to thioredoxins including TRXh5, which is required for redox control of the transcriptional immune regulator NPR1. TRXh 5 binding activates the NB-LRR-type immune receptor LOV1, facilitating necrotrophic exploitation of host cell death by C. victoriae ${ }^{29}$.

In conclusion, although information for the vast majority of pathogen effectors, particularly of filamentous pathogens, is still lacking, effector molecules are highly versatile. Clearly, recently uncovered functions revealed that virulence effectors, despite the finding that they converge onto pivotal elements of the plant immune system ${ }^{30}$, can deregulate any step of immunity in any cellular compartment (Figure 1, Table 1). 


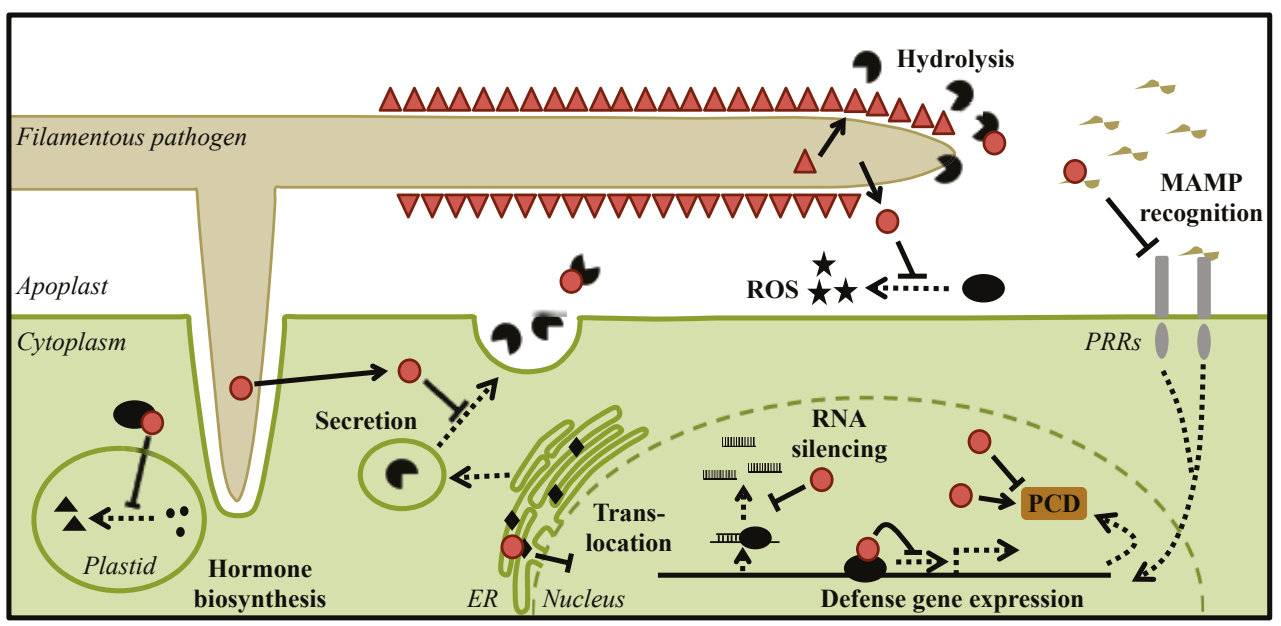

FIGURE 1 | Filamentous pathogen effectors deregulate host immunity in various host subcellular compartments. Pathogens secrete effectors (red symbols) to deregulate plant immunity (see text for details). Whereas one group of effectors (red circles) interacts with host targets that act in immunity (black shapes), another group of effectors (red triangles) acts in self-defense to protect the pathogen from host-derived antimicrobials.

\section{Endophytes and mutualists use effectors to suppress host immunity too}

Like pathogens, commensalistic endophytes and mutualists develop intimate host plant associations. During initiation of such symbioses, PRRs continue to perceive MAMPs. Consequently, similar to pathogens, endophytes and mutualists are recipients of immune responses. However, the precise role and fate of host immunity in the establishment of symbiosis has remained enigmatic.

The root endophyte Serendipita indica has a wide host range and induces enhanced growth and stress resistance in colonized hosts. Rather than evading host detection, the fungus actively suppresses immunity ${ }^{31}$. During early biotrophic growth at the onset of symbiosis, about $10 \%$ of the transcriptome encodes putative effector proteins ${ }^{32}$. At later growth stages the fungus requires host cell death for further colonization, thus resembling hemibiotrophic pathogens such as Zymoseptoria tritici and M. oryzae. Like C. fulvum, these latter species utilize LysM effectors to suppress immune responses ${ }^{3,5,6}$. S. indica carries an expanded LysM domain-containing effector repertoire that may similarly act in immune suppression $^{32}$.

Effector-like proteins are also encoded by genomes of other mutualists ${ }^{33-35}$. The ectomycorrhiza Laccaria bicolor genome encodes hundreds of small, secreted proteins, several of which are only expressed in symbiotic tissues. Of these, MiSSP7 was shown to translocate to the nucleus of poplar host cells to stabilize the JAZ6 protein and repress 
jasmonate signaling ${ }^{34,36}$. Likewise, the ectomycorrhiza Tuber melanosporum expresses 125 cysteine-rich small secreted proteins, including a LysM effector, which are highly upregulated during symbiosis ${ }^{35}$.

TABLE 1 | Effectors of filamentous plant-associated microbes for which molecular virulence targets were identified

\begin{tabular}{|c|c|c|c|c|}
\hline Effector & Origin & Target & Function & Reference \\
\hline BEC4 & $\begin{array}{l}\text { Blumeria graminis f.sp. } \\
\text { hordei }\end{array}$ & ARF-GAP proteins & Interference with host vesicle trafficking & 41 \\
\hline Avr2 & Cladosporium fulvum & Cysteine proteases & Cysteine protease inhibition & 10,42 \\
\hline Avr4 & Cladosporium fulvum & Chitin & Hyphal protection & 43 \\
\hline Ecp6 & Cladosporium fulvum & Chitin & Perturbation chitin-triggered immunity & 3 \\
\hline CfTom 1 & Cladosporium fulvum & a-tomatine & Detoxification & 44 \\
\hline Victorin & Cochliobolus victoria & TRX-h5 & Induction of LOV1-mediated cell death & 29 \\
\hline SP7 & Rhizophagus irregularis & ERF19 & Deregulation of host gene expression & 40 \\
\hline HaRxL44 & $\begin{array}{l}\text { Hyaloperonospora } \\
\text { arabidopsidis }\end{array}$ & MED19a & Interference with SA-triggered immunity & 23 \\
\hline MiSSP7 & Laccaria bicolor & JAZ6 & Deregulation of host gene expression & 33 \\
\hline AvrPiz-t & Magnaporthe oryzae & $\begin{array}{l}\text { RING E3 ubiquitin } \\
\text { ligase APIP6 }\end{array}$ & $\begin{array}{l}\text { Suppression of MAMP-triggered } \\
\text { immunity }\end{array}$ & 20 \\
\hline Slp1 & Magnaporthe oryzae & Chitin & Perturbation chitin-triggered immunity & 6 \\
\hline MfAvr4 & Pseudocercospora fijiensis & Chitin & Hyphal protection & 45 \\
\hline Mg1LysM & Zymoseptoria tritici & Chitin & Hyphal protection & 5 \\
\hline Mg3LysM & Zymoseptoria tritici & Chitin & Perturbation chitin-triggered immunity & 5 \\
\hline Avr3a & Phytophthora infestans & CMPG1 & E3 ligase stabilization & 27 \\
\hline Avrblb2 & Phytophthora infestans & C14 protease & Suppression of protease secretion & 14 \\
\hline EPI1 & Phytophthora infestans & Serine proteases & Inhibition of serine proteases & 46 \\
\hline EPI10 & Phytophthora infestans & Serine proteases & Inhibition of serine proteases & 47 \\
\hline EPIC1 & Phytophthora infestans & Cysteine proteases & Inhibition of cysteine proteases & 11,48 \\
\hline EPIC2B & Phytophthora infestans & Cysteine proteases & Inhibition of cysteine proteases & 11,48 \\
\hline PexRD2 & Phytophtora infestans & MAPKKK $\varepsilon$ & Suppression of host cell death & 28 \\
\hline Pi03192 & Phytophthora infestans & NTP1, NTP2 & $\begin{array}{l}\text { Suppression of transcription factor } \\
\text { relocation }\end{array}$ & 24 \\
\hline GIP1 & Phytophthora sojae & $\beta$-1,3-glucanases & Glucanase inhibition & 49 \\
\hline RTP1p & Uromyces fabae/U. striatus & Proteases & Protease inhibition & 50 \\
\hline Cmu1 & Ustilago maydis & $\mathrm{Cm} 2$ & Interference with SA biosynthesis & 21 \\
\hline Pep1 & Ustilago maydis & POX12 & $\begin{array}{l}\text { Inhibition of peroxidase-mediated ROS } \\
\text { production }\end{array}$ & 17 \\
\hline Pit2 & Ustilago maydis & $\begin{array}{l}\mathrm{CP} 2, \mathrm{CP} 1 \mathrm{~A} / \mathrm{B} \\
\mathrm{XCP} 2 \text { proteases }\end{array}$ & Cysteine protease inhibition & 15 \\
\hline Tin2 & Ustilago maydis & TmTTK1 & Control of anthocyanin biosynthesis & 22 \\
\hline
\end{tabular}


It was recently shown that arbuscular endomycorrhizal fungi produce lipochitooligosaccharide mycorrhizal (Myc) factors that stimulate root growth and branching to initiate symbiosis ${ }^{37}$. Similar to endophytes and ectomycorrhiza, arbuscular endomycorrhiza secrete effector-like proteins during symbiotic interactions ${ }^{38-40}$. The genome of Rhizophagus irregularis encodes a family of CRN-like proteins that are abundantly found in plant pathogenic Phytophthora spp. ${ }^{39}$. R. irregularis was furthermore found to encode an effector that interacts with the pathogenesis-related ethylene-responsive transcription factor 19 (ERF19) in the host nucleus to promote mycorrhization, potentially by counteracting MAMP-induced host defense responses that are regulated by ERF1940.

Collectively, these findings suggest that symbiotic associations that include endophytism, mutualism and parasitism form a continuum in which effectors play essential roles (Table 1).

\section{Effectors act in self-defense and competition}

The ability to establish symbiosis evolved multiple times in microbes, presumably from saprotrophism, and many plant pathogens still display saprotrophic life stages. Saprotrophs generally reside within the soil where they feed on decaying organic matter in the presence of a rich microbiota. In this environment, microbial competition as well as co-operation occurs (Figure 2). Threats are posed by (myco)parasites and competitors that produce antibiotics with specific or broad-spectrum activities. Consequently, microbes require molecules for self-defense and interaction with other microbiome partners.

Similar to infected plants, many mycoparasites secrete hydrolytic enzymes including proteases, chitinases and glucanases to target fungal cell walls. Presumably, chitin-binding effectors that protect hyphal cell walls against plant-derived chitinases similarly protect against mycoparasite-derived chitinases, which may explain abundant LysM effector catalogs of non-pathogenic fungi ${ }^{51,52}$. As LysM domains occur in peptidoglycan-binding proteins of various origins, LysM effector homologs that bind non-chitin substrates likely occur. Indeed, a plant pathogen LysM effector that binds bacterial cell walls was characterized (Kombrink and Thomma, unpublished data), potentially implicating this effector in bacterial competition or protection against bacterial mycoparasites. Genome analyses furthermore revealed that saprotrophic species encode abundant catalogs of small, secreted proteins that resemble pathogen effector catalogs $\mathrm{s}^{52-55}$. Although these potential effectors are poorly studied, one such effector, CipC, was implicated in competition with bacteria in Aspergillus spp. ${ }^{55,56}$. The genome of the ubiquitous saprophyte and opportunistic mammalian pathogen $A$. fumigatus encodes several effector proteins ${ }^{57}$. However, since the vast majority of fungi that cause disease in animals are soil saprophytes that opportunistically infect their hosts, to which they are not highly adapted, it has been speculated that infection does not rely on the activity of effectors ${ }^{58}$. Rather, their effectors 
are thought to be required for saprophytic survival ${ }^{58}$. Nevertheless, effectors that evolved to enable saprophytic survival may be co-opted for opportunistic infection as well.

Likely, competition between plant-associated microbes also occurs within hosts, although perhaps to a lesser extent than in soil due to reduced species diversity. Indeed, the second most abundantly in planta-expressed gene of the fungal endophyte Epichloë festucae encodes a secreted antifungal protein ${ }^{59}$. Thus, effector homologs may play crucial roles in microbial competition in a broad spectrum of environments.

\section{Do pathogens shape local microbiomes?}

For various types of multicellular organisms it is increasingly recognized that their microbiome, i.e. the community of microbes that thrives in, on, or immediately near the organism, greatly influences its performance ${ }^{60}$. For plants, it has been particularly well documented that the rhizosphere microbiota affects plant growth and stress tolerance. In addition, the importance of the phyllosphere microbiota is increasingly recognized ${ }^{61}$. These microbiota comprise members that provide direct as well as indirect pathogen protection through antibiosis and induced immunity, respectively. Whereas soil types have a major impact on root inhabiting bacterial community compositions on Arabidopsis, host genotypes were reported to only have a minor impact ${ }^{62,63}$. In contrast, different Arabidopsis accessions were found to harbor different phyllosphere communities and several host genetic mutations were found to perturb the microbiota composition, demonstrating that host genetic factors shape the associated microbiota ${ }^{64}$. It is less clear, however, whether plants evolved to actively recruit phyllosphere communities. Potentially, plants recruit founder species that further shape local microbiomes through inter-microbe interactions ${ }^{61}$. Such interactions may require effectors. Considering that plant factors control the composition of the microbiota, microbiome members may utilize effectors to modulate hosts and control competitors indirectly. Additionally, manipulation of host metabolism could even establish microbial cooperation (Figure 2). Although not immediately addressing inter-microbial interactions, an insect-transmitted phytoplasma was recently shown to utilize an effector to alter floral development of host plants, converting them into vegetative tissues that attract leafhopper vectors ${ }^{65}$. This represents a striking example of the exploitation of effector activity to influence compositions of the local biome. Similarly, the rust fungus Puccinia monoica induces floral mimicry in the host Boechera stricta to enhance its reproduction and spore dispersal by insects ${ }^{66}$.

Considering the importance of the microbiome for the ability of plants to withstand pathogen infection, it is conceivable that pathogens evolved to affect host microbiomes, possibly through effector activities (Figure 2). 


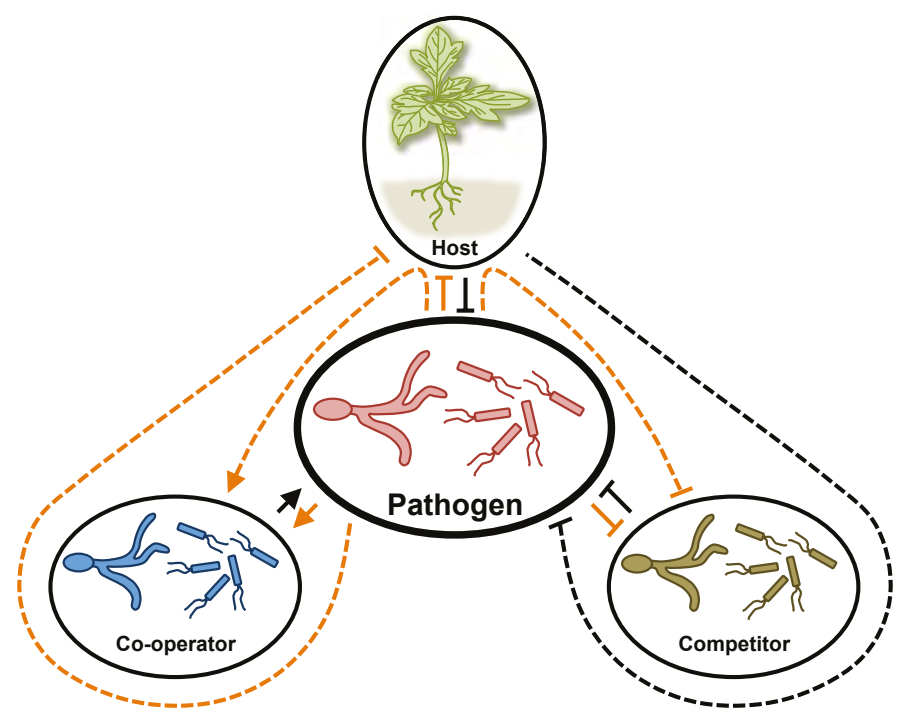

FIGURE 2 | How pathogens influence the local biota by exploiting effector activities. The interaction between microbial pathogens and plant hosts occurs in environments that contain additional microbiome partners that can negatively (competition) or positively (co-operation) impact the pathogen as well as the host. Consequently, the pathogen and host may target each other directly (solid lines) as well as indirectly (dotted lines). Likely, pathogens exploit effector activities (orange lines) to not only directly modulate their hosts, but also to influence the local microbiota that can impact the outcome of the interaction with their hosts.

\section{Different mechanisms drive evolution of effector repertoires}

Mechanisms underlying genome plasticity and evolution have been intensely studied, especially for plant pathogens. As genomes are structured and not just a random sequence of genes, effector genes are often found in dynamic genomic compartments, such as gene-sparse regions, subtelomeric regions or conditionally dispensable (pathogenicity) chromosomes ${ }^{67}$. For example, effector localization in gene-sparse regions was recorded for the endophyte $S$. indica ${ }^{32}$, while in the saprophyte $N$. crassa genes encoding small-secreted proteins are found in subtelomeric regions ${ }^{53}$. Genetic plasticity in such compartments is governed by diverse mechanisms including recombination and activity of transposable elements. A direct implication of genomic rearrangement in the evolution of fungal aggressiveness was shown for the vascular wilt fungus Verticillium dahliae, leading to the emergence of lineage-specific regions that are enriched for virulence effectors ${ }^{68}$. High genetic variability in effector genes enables rapid evolutionary processes. The importance of dynamic genome compartments for accelerated gene evolution was underlined in the specialization of $P$. infestans after the host jump that separated this species from related species. Uneven evolutionary rates across the genome occur, with in planta-induced genes residing in fast evolving compartments ${ }^{69}$. In turn, effector specialization can lead 
to diversification and speciation in pathogen lineages ${ }^{13}$. In this manner, effectors can determine microbial niches. Moreover, composition of effector catalogs can dictate microbial lifestyles. For example, the leaf epiphyte and antagonist of powdery mildews Pseudozyme flucculosa lost its ability to parasitize plants like its smut fungi relatives due to loss of virulence effectors ${ }^{70}$. However, the biocontrol agent has acquired other effectors that are not found in the smut relatives that may have shaped its current lifestyle ${ }^{70}$. These findings suggest that effector catalogs evolve via different mechanisms and that their composition influences a microbe's lifestyle in a given environment.

\section{Experimental way forward}

The interaction between pathogenic (filamentous) microbes and the organisms they encounter in their niches, either while colonizing the host or during free-living stages in the environment, is poorly understood. An extensive characterization of the complex microbial communities in such niches may lead to a better understanding of the interactions that take place beyond the direct interaction between pathogen and host. Detailed transcriptome analyses may lead to the identification of particular triggers of effector gene expression derived from microbial co-inhabitants, and may hint towards functions in inter-microbial interactions ${ }^{71,72}$ that can subsequently be tested for in targeted analysis to reveal components that either promote or inhibit other microbes ${ }^{52}$.

\section{Conclusions}

Although a paradigm in plant pathology dictates that existence of disease requires the interaction of a virulent pathogen with a susceptible host in a favorable environment, plant-microbe interactions are mostly studied as one-on-one relationships. However, in addition to host immune responses, pathogenic microbes continuously encounter other microbes that include competitors and mycoparasites that need to be dealt with simultaneously. Importantly, findings for pathogenic microbes can be extrapolated to other types of symbioses as well. After all, irrespective of the type of symbiosis, the interest of the microbial partner is merely to exploit the host for nutrition and shelter. This may also explain the thin line that is regularly observed between the different types of symbioses $32,33,73,74$. In all types of symbioses, the microbial partner needs to suppress host immune responses and ward off microbial antagonists. Using effectors as probes, further critical processes in host colonization will be uncovered, leading to enhanced understanding of the biology of microbes that aim to establish symbioses. 


\section{Acknowledgements}

The authors acknowledge support by the Research Council for Earth and Life sciences (ALW) of the Netherlands Organization for Scientific Research (NWO) (Grant No. 865.11.003), thank Melvin Bolton, Andrea Sánchez-Vallet, and Ronnie de Jonge for critically reading the manuscript.

\section{References}

1 Dodds, P. N. \& Rathjen, J. P. Plant immunity: towards an integrated view of plant-pathogen interactions. Nat. Rev. Genet. 11, 539-548, doi:10.1038/nrg2812 (2010).

2 Thomma, B. P. H. J., Nürnberger, T. \& Joosten, M. H. A. J. Of PAMPs and effectors: the blurred PTIETI dichotomy. Plant Cell 23, 4-15, doi:10.1105/ tpc110.082602 (2011).

3 de Jonge, R. et al. Conserved fungal LysM effector Ecp6 prevents chitin-triggered immunity in plants. Science 329, 953-955, doi:10.1126/science.1190859 (2010).

4 Kombrink, A., Sánchez-Vallet, A. \& Thomma, B. P. H. J. The role of chitin detection in plant-pathogen interactions. Microb. Infect. 13, 1168-1176, doi:10.1016/j.micinf.2011.07.010 (2011).

5 Marshall, R. et al. Analysis of two in planta expressed LysM effector homologs from the fungus Mycosphaerella graminicola reveals novel functional properties and varying contributions to virulence on wheat. Plant Physiol. 156, 756-769, doi:10.1104/ pp.111.176347 (2011).

6 Mentlak, T. A. et al. Effector-mediated suppression of chitin-triggered immunity by Magnaporthe oryzae is necessary for rice blast disease. Plant Cell 24, 322 335, doi:10.1105/tpc.111.092957 (2012).

7 Sánchez-Vallet, A. et al. Fungal effector Ecp6 outcompetes host immune receptor for chitin binding through intrachain LysM dimerization. eLife 2, e00790, doi:10.7554/eLife.00790 (2013).

8 Liu, T. et al. Chitin-induced dimerization activates a plant immune receptor. Science 336, 1160-1164, doi:10.1126/science.1218867 (2012).

9 Hayafune, M. et al. Chitin-induced activation of immune signaling by the rice receptor CEBiP relies on a unique sandwich-type dimerization. Proc. Natl. Acad. Sci. USA 111, E404-413, doi:10.1073/ pnas.1312099111 (2014).
10 van Esse, H. P. et al. The Cladosporium fulvum virulence protein Avr2 inhibits host proteases required for basal defense. Plant cell 20, 1948-1963, doi:10.1105/tpc.108.059394 (2008).

11 Song, J. et al. Apoplastic effectors secreted by two unrelated eukaryotic plant pathogens target the tomato defense protease Rcr3. Proc. Natl. Acad. Sci. USA 106, 1654-1659, doi:10.1073/pnas.0809201106 (2009).

12 Lozano-Torres, J. L. et al. Dual disease resistance mediated by the immune receptor $\mathrm{Cf}-2$ in tomato requires a common virulence target of a fungus and a nematode. Proc. Natl. Acad. Sci. USA 109, 1011910124, doi:10.1073/pnas.1202867109 (2012).

13 Dong, S. et al. Effector specialization in a lineage of the Irish potato famine pathogen. Science 343, 552555, doi:10.1126/science.1246300 (2014).

14 Bozkurt, T. O. et al. Phytophthora infestans effector AVRblb2 prevents secretion of a plant immune protease at the haustorial interface. Proc. Natl. Acad. Sci. USA 108, 20832-20837, doi:10.1073/ pnas.1112708109 (2011).

15 Mueller, A. N., Ziemann, S., Treitschke, S., Assmann, D. \& Doehlemann, G. Compatibility in the Ustilago maydis-maize interaction requires inhibition of host cysteine proteases by the fungal effector Pit2. PLoS Pathog. 9, e1003177, doi:10.1371/journal. ppat.1003177 (2013).

16 van der Linde, K. et al. A maize cystatin suppresses host immunity by inhibiting apoplastic cysteine proteases. Plant Cell 24, 1285-1300, doi:10.1105/ tpc.111.093732 (2012).

17 Hemetsberger, C., Herrberger, C., Zechmann, B., Hillmer, M. \& Doehlemann, G. The Ustilago maydis effector Pep1 suppresses plant immunity by inhibition of host peroxidase activity. PLoS Pathog. 8, e1002684, doi:10.1371/journal.ppat.1002684 (2012). 
18 Petre, B. \& Kamoun, S. How do filamentous pathogens deliver effector proteins into plant cells? PLoS Biol. 12, e1001801, doi:10.1371/journal. pbio.1001801 (2014).

19 Khang, C. H. et al. Translocation of Magnaporthe oryzae effectors into rice cells and their subsequent cell-to-cell movement. Plant Cell 22, 1388-1403, doi:10.1105/tpc.109.069666 (2010).

20 Park, C. H. et al. The Magnaporthe oryzae effector AvrPiz-t targets the RING E3 ubiquitin ligase APIP6 to suppress pathogen-associated molecular pattern-triggered immunity in rice. Plant Cell 24 4748-4762, doi:10.1105/tpc.112.105429 (2012).

21 Djamei, A. et al. Metabolic priming by a secreted fungal effector. Nature 478, 395-398, doi:10.1038/ nature10454 (2011).

22 Tanaka, S. et al. A secreted Ustilago maydis effector promotes virulence by targeting anthocyanin biosynthesis in maize. elife 3, e01355, doi:10.7554/ elife.01355 (2014).

23 Caillaud, M. C. et al. A downy mildew effector attenuates salicylic acid-triggered immunity in Arabidopsis by interacting with the host mediator complex. PLoS Biol. 11, e1001732, doi:10.1371/ journal.pbio.1001732 (2013).

24 McLellan, $H$. et al. An RxLR effector from Phytophthora infestans prevents re-localisation of two plant NAC transcription factors from the endoplasmic reticulum to the nucleus. PLOS Path. 9, e1003670, doi:10.1371/journal.ppat.1003670 (2013).

25 Qiao, Y. et al. Oomycete pathogens encode RNA silencing suppressors. Nature Genet. 45, 330-333, doi:10.1038/ng.2525 (2013).

26 Weiberg, A. et al. Fungal small RNAs suppress plant immunity by hijacking host RNA interference pathways. Science 342, 118-123, doi:10.1126/ science.1239705 (2013).

27 Bos, J. I. et al. Phytophthora infestans effector AVR3a is essential for virulence and manipulates plant immunity by stabilizing host E3 ligase CMPG1. Proc. Natl. Acad. Sci. USA 107, 9909-9914, doi:10.1073/pnas.0914408107 (2010).

28 King, S. R. et al. Phytophthora infestans RXLR effector PexRD2 interacts with host MAPKKK epsilon to suppress plant immune signaling. Plant Cell 26, 1345-1359, doi:10.1105/tpc.113.120055 (2014).
29 Lorang, J. et al. Tricking the guard: exploiting plant defense for disease susceptibility. Science 338, 659662, doi:10.1126/science.1226743 (2012).

30 Mukhtar, M. S. et al. Independently evolved virulence effectors converge onto hubs in a plant immune system network. Science 333, 596-601, doi:10.1126/science.1203659 (2011).

31 Jacobs, S. et al. Broad-spectrum suppression of innate immunity is required for colonization of Arabidopsis roots by the fungus Piriformospora indica. Plant Phys. 156, 726-740, doi:10.1104/ pp.111.176446 (2011).

32 Zuccaro, A. et al. Endophytic life strategies decoded by genome and transcriptome analyses of the mutualistic root symbiont Piriformospora indica. PLoS Pathog. 7, doi:10.1371/journal.ppat.1002290 (2011).

33 Plett, J. M. \& Martin, F. Blurred boundaries: lifestyle lessons from ectomycorrhizal fungal genomes. Trends Genet. 27, 14-22, doi:10.1016/j. tig.2010.10.005 (2011).

34 Plett, J.M.etal. A secreted effector protein of Laccaria bicolor is required for symbiosis development. Curr. Biol. 21, 1197-1203, doi:10.1016/j.cub.2011.05.033 (2011).

35 Martin, F. et al. Perigord black truffle genome uncovers evolutionary origins and mechanisms of symbiosis. Nature 464, 1033-1038, doi:10.1038/ nature08867 (2010).

36 Plett, J. M. et al. Effector MiSSP7 of the mutualistic fungus Laccaria bicolor stabilizes the Populus JAZ6 protein and represses jasmonic acid (JA) responsive genes. Proc. Natl. Acad. Sci. USA 111, 8299-8304, doi:10.1073/pnas.1322671111 (2014).

37 Maillet, F. et al. Fungal lipochitooligosaccharide symbiotic signals in arbuscular mycorrhiza. Nature 469, 58-U1501, doi:10.1038/nature09622 (2011).

38 Tisserant, E. et al. Genome of an arbuscular mycorrhizal fungus provides insight into the oldest plant symbiosis. Proc. Natl. Acad. Sci. USA 110, 20117-20122, doi:10.1073/pnas.1313452110 (2013).

39 Lin, K. et al. Single nucleus genome sequencing reveals high similarity among nuclei of an endomycorrhizal fungus. PLoS Genet. 10, e1004078, doi:10.1371/journal.pgen.1004078 (2014).

40 Kloppholz, S., Kuhn, H. \& Requena, N. A secreted fungal effector of Glomus intraradices promotes symbiotic biotrophy. Curr. Biol. 21, 1204-1209, doi:10.1016/j.cub.2011.06.044 (2011). 
41 Schmidt, S. M. et al. Interaction of a Blumeria graminis f. sp. hordei effector candidate with a barley ARF-GAP suggests that host vesicle trafficking is a fungal pathogenicity target. Mol. Plant Pathol. 15, 535-549, doi:10.1111/mpp.12110 (2014).

42 Shabab, M. et al. Fungal effector protein AVR2 targets diversifying defense-related cys proteases of tomato. Plant Cell 20, 1169-1183, doi:10.1105/ tpc.107.056325 (2008).

43 van den Burg, H. A., Harrison, S. J., Joosten, M. H., Vervoort, J. \& De Wit, P. J. Cladosporium fulvum Avr4 protects fungal cell walls against hydrolysis by plant chitinases accumulating during infection. Mol. Plant-Microbe Interact. 19, 1420-1430 (2006).

44 Ökmen, B. et al. Detoxification of alpha-tomatine by Cladosporium fulvum is required for full virulence on tomato. New Phytol. 198, 1203-1214, doi:10.1111/nph.12208 (2013).

45 Stergiopoulos, I. et al. Tomato Cf resistance proteins mediate recognition of cognate homologous effectors from fungi pathogenic on dicots and monocots. Proc. Natl. Acad. Sci. USA 107, 7610 7615, doi:10.1073/pnas.1002910107 (2010).

46 Tian, M., Huitema, E., Da Cunha, L., Torto-Alalibo, T. \& Kamoun, S. A Kazal-like extracellular serine protease inhibitor from Phytophthora infestans targets the tomato pathogenesis-related protease P69B. J. Biol. Chem. 279, 26370-26377, doi:10.1074/ jbc.M400941200 (2004).

47 Tian, M., Benedetti, B. \& Kamoun, S. A Second Kazal-like protease inhibitor from Phytophthora infestans inhibits and interacts with the apoplastic pathogenesis-related protease P69B of tomato. Plant Phys. 138, 1785-1793, doi:10.1104/ pp.105.061226 (2005).

48 Tian, M. et al. A Phytophthora infestans cystatinlike protein targets a novel tomato papain-like apoplastic protease. Plant Phys. 143, 364-377, doi:10.1104/pp.106.090050 (2007).

49 Rose, J. K., Ham, K. S., Darvill, A. G. \& Albersheim, P. Molecular cloning and characterization of glucanase inhibitor proteins: coevolution of a counterdefense mechanism by plant pathogens. Plant Cell 14, 1329-1345 (2002).

50 Pretsch, K. et al. The rust transferred proteins-a new family of effector proteins exhibiting protease inhibitor function. Mol. Plant Pathol. 14, 96-107, doi:10.1111/j.1364-3703.2012.00832.x (2013).
51 Kubicek, C. P.et al. Comparative genome sequence analysis underscores mycoparasitism as the ancestral life style of Trichoderma. Gen. Biol. 12, R40, doi:10.1186/gb-2011-12-4-r40 (2011).

52 Kombrink, A. \& Thomma, B. P. H. J. LysM effectors: secreted proteins supporting fungal life. PLoS Pathog. 9, e1003769, doi:10.1371/journal. ppat.1003769 (2013).

53 Kasuga, T., Mannhaupt, G. \& Glass, N. L. Relationship between phylogenetic distribution and genomic features in Neurospora crassa. PLoS ONE 4, e5286, doi:10.1371/journal.pone.0005286 (2009).

54 Druzhinina, I. S., Shelest, E. \& Kubicek, C. P. Novel traits of Trichoderma predicted through the analysis of its secretome. FEMS Microbiol. Let. 337, 1-9, doi:10.1111/j.1574-6968.2012.02665.x (2012).

55 Suh, M. J. et al. Development stage-specific proteomic profiling uncovers small, lineage specific proteins most abundant in the Aspergillus fumigatus conidial proteome. Prot. Sci. 10, 30, doi:10.1186/1477-5956-10-30 (2012).

56 Melin, P., Schnurer, J. \& Wagner, E. G. Proteome analysis of Aspergillus nidulans reveals proteins associated with the response to the antibiotic concanamycin A, produced by Streptomyces species. Mol. Genet. Genom. 267, 695-702, doi:10.1007/s00438-002-0695-0 (2002).

57 Wartenberg, D. et al. Secretome analysis of Aspergillus fumigatus reveals asp-hemolysin as a major secreted protein. Int. J. Med. Microbiol. 301, 602-611, doi:10.1016/j.jjmm.2011.04.016 (2011).

58 Lowe, R. G. \& Howlett, B. J. Indifferent, affectionate, or deceitful: lifestyles and secretomes of fungi. PLoS Pathog. 8, e1002515, doi:10.1371/journal. ppat.1002515 (2012).

59 Ambrose, K. V. \& Belanger, F. C. SOLiD-SAGE of endophyte-infected red fescue reveals numerous effects on host transcriptome and an abundance of highly expressed fungal secreted proteins. PLOS ONE 7, e53214, doi:10.1371/journal.pone.0053214 (2012).

60 Ezenwa, V. O., Gerardo, N. M., Inouye, D. W., Medina, M. \& Xavier, J. B. Microbiology. Animal behavior and the microbiome. Science 338, 198199, doi:10.1126/science.1227412 (2012).

61 Vorholt, J. A. Microbial life in the phyllosphere. Nature Rev. Microbiol. 10, 828-840, doi:10.1038/ nrmicro2910 (2012). 
62 Bulgarelli, D. et al. Revealing structure and assembly cues for Arabidopsis root-inhabiting bacterial microbiota. Nature 488, 91-95, doi:10.1038/nature11336 (2012).

63 Lundberg, D. S. et al. Defining the core Arabidopsis thaliana root microbiome. Nature 488, 86-90, doi:10.1038/nature11237 (2012).

64 Bodenhausen, N., Bortfeld-Miller, M., Ackermann, M. \& Vorholt, J. A. A synthetic community approach reveals plant genotypes affecting the phyllosphere microbiota. PLoS Genet. 10, e1004283, doi:10.1371/journal.pgen.1004283 (2014).

65 MacLean, A. M. et al. Phytoplasma effector SAP54 hijacks plant reproduction by degrading MADSbox proteins and promotes insect colonization in a RAD23-dependent manner. PLoS Biol. 12, e1001835, doi:10.1371/journal.pbio.1001835 (2014).

66 Cano, L. M. et al. Major transcriptome reprogramming underlies floral mimicry induced by the rust fungus Puccinia monoica in Boechera stricta. PLOS ONE 8, e75293, doi:10.1371/journal. pone.0075293 (2013).

67 Raffaele, S. \& Kamoun, S. Genome evolution in filamentous plant pathogens: why bigger can be better. Nature Rev. Microbiol. 10, 417-430, doi:10.1038/nrmicro2790 (2012).

68 de Jonge, R. et al. Extensive chromosomal reshuffling drives evolution of virulence in an asexual pathogen. Gen. Res. 23, 1271-1282, doi:10.1101/gr.152660.112 (2013).
69 Raffaele, S. et al. Genome evolution following host jumps in the Irish potato famine pathogen lineage. Science 330, 1540-1543, doi:10.1126/ science.1193070 (2010).

70 Lefebvre, F. et al. The transition from a phytopathogenic smut ancestor to an anamorphic biocontrol agent deciphered by comparative whole-genome analysis. Plant Cell 25, 1946-1959, doi:10.1105/tpc.113.113969 (2013).

71 Mela, F. et al. Dual transcriptional profiling of a bacterial/fungal confrontation: Collimonas fungivorans versus Aspergillus niger. ISME J. 5, 1494-1504, doi:10.1038/ismej.2011.29 (2011).

72 Mathioni, S. M. et al. Transcriptomics of the rice blast fungus Magnaporthe oryzae in response to the bacterial antagonist Lysobacter enzymogenes reveals candidate fungal defense response genes. PLOS ONE 8, e76487, doi:10.1371/journal. pone.0076487 (2013).

73 Delaye, L., García-Guzmán, G. \& Heil, M. Endophytes versus biotrophic and necrotrophic pathogens-are fungal lifestyles evolutionarily stable traits? Fungal Div. 60, 125-135, doi:10.1007/ s13225-013-0240-y (2013).

74 Malcolm, G. M., Kuldau, G. A., Gugino, B. K. \& Jimenez-Gasco Mdel, M. Hidden host plant associations of soilborne fungal pathogens: an ecological perspective. Phytopathol. 103, 538544, doi:10.1094/PHYTO-08-12-0192-LE (2013). 


\title{
Chapter 3
}

\author{
The fungal effector protein Ecp6 \\ interferes with chitin perception \\ in the model plant Arabidopsis thaliana
}

Hanna Rovenich', Elena K. Petutschnig', Jan Erwig'2, Bart P.H.J. Thomma', Volker Lipka²

'Laboratory of Phytopathology, Wageningen University, Droevendaalsesteeg 1, 6708 PB Wageningen, The Netherlands; ${ }^{2}$ Department of Plant Cell Biology, Schwann-Schleiden Centre, Georg-August-University Göttingen, Julia-Lermontowa-Weg 3, 37077 Göttingen, Germany 


\begin{abstract}
Fungal microbes utilize effector proteins to suppress chitin-triggered immunity during plant colonization. The tomato leaf mold fungus Cladosporium fulvum secretes the lysin motif (LysM)-containing effector protein Ecp6. Ecp6 has the capacity to outcompete plant chitin receptors through cooperative high affinity binding of chitin substrates between two of its three LysM domains. Additionally, the singular second LysM domain (LysM2) can perturb chitin responses in tomato through a yet unknown mechanism. Due to its relatively low affinity for chitin, it has been hypothesized that LysM2 interferes with chitin receptor dimerization required for the activation of immunity. To further investigate Ecp6 functionality, we utilized the model plant Arabidopsis thaliana for which chitin perception has been intensely studied. Here, we show that Ecp6 suppresses chitin-mediated immune responses in A. thaliana. While Ecp6 does not suppress phosphorylation of the LysM receptor kinase AtCERK1, it affects internalization of the LysM receptor kinase AtLYK5 in a ligand-dependent manner. These findings suggest that, in A. thaliana, Ecp6 affects chitin perception by targeting chitin receptors through a yet unknown mechanism.
\end{abstract}




\section{Introduction}

Chitin is one of the most abundant carbohydrates in nature, and represents a major component of fungal cell walls. During early stages of host colonization, invasive hyphae of filamentous microbes encounter host-derived hydrolytic enzymes, such as chitinases, that release chitin oligosaccharides from fungal cell walls ${ }^{1}$. Recognition of chitin oligosaccharides by host receptors prompts the secretion of antimicrobial compounds and toxins, as well as the release of additional cell wall degrading enzymes such as endochitinases that hydrolyze fungal hyphae. These chitin-dependent immune responses are detrimental to fungal growth and may eventually halt host invasion ${ }^{2,3}$.

In rice (Oryza sativa), chitin recognition is mediated by the lysin motif (LysM)-containing Chitin Elicitor Binding Protein (OsCEBiP), which lacks an intracellular kinase domain ${ }^{4}$. OsCEBiP dimerizes upon ligand binding and forms a tetrameric receptor complex with Chitin Elicitor Receptor Kinase 1 (OSCERK1) to initiate chitin signalling ${ }^{5,6}$. As in rice, Arabidopsis thaliana (hereafter Arabidopsis) CERK1 is required for chitin signalling ${ }^{7,8}$. However, unlike OsCERK1, AtCERK1 directly binds long chain chitin fragments via its second LysM domain with a relatively low affinity ${ }^{9-11}$. In addition to AtCERK1, the LysM receptor kinases AtLYK4 and AtLYK5, both of which lack active kinase domains, were identified as chitin-binding proteins ${ }^{10}$. AtLYK5 was shown to interact with AtCERK1 in a ligand-dependent manner, required for AtCERK1 dimerization and phosphorylation ${ }^{12}$. Due to its higher affinity for longer chain chitin oligomers, AtLYK5 was proposed as the primary chitin receptor in Arabidopsis. Remarkably, despite its high affinity for chitin oligomers $^{13}$, the Arabidopsis orthologue of OsCEBiP, LysM domain protein 2 (AtLYM2), is not involved in AtCERK1-mediated chitin responses but rather modulates plasmodesmata conductivity in a AtCERK1-independent manner ${ }^{14}$.

To overcome or bypass chitin-triggered immune responses, filamentous microbes employ various strategies, including cell wall modifications and the secretion of LysM-containing effector proteins that suppress chitin-triggered immune responses ${ }^{15-17}$. Moreover, effector proteins can shield cell wall chitin, thereby preventing hydrolysis by plant chitinases. For example, the tomato leaf mold fungus Cladosporium fulvum produces avirulence protein 4 (Avr4), which binds chitin through its invertebrate chitin-binding domain (CBM14) $)^{18,19}$. While Avr4 protects invasive hyphae, it is not able to perturb chitin-induced immune responses.

In addition to Avr4, C. fulvum secretes the LysM effector protein Ecp6. Ecp6 compromises chitin-induced immune responses by chitin binding with ultra-high (pM) affinity through intramolecular LysM domain dimerization, thereby outcompeting host receptors ${ }^{20}$. Interestingly, the singular LysM2 domain of Ecp6, which is not involved in intramolecular chitin binding, retains the capacity to perturb chitin-induced immune responses ${ }^{20,21}$. Due to its lower chitin affinity, it is unlikely that LysM2 deregulates chitin-triggered 
immunity by chitin fragment sequestration ${ }^{22}$. Therefore, it has been hypothesized that LysM2 may interfere with the chitin-induced host receptor dimerization that is required for the activation of immune signalling ${ }^{3,20}$. Since the mechanisms underlying chitin recognition have not been identified in the C. fulvum host plant tomato, we made use of the Arabidopsis model system to study the potential role of Ecp6 in the perturbation of chitin receptor complex activation.

\section{Results and Discussion}

When applied to leaf discs of Arabidopsis ecotypes Col-0 or WS-4, polymeric chitin triggers the production of reactive oxygen species (ROS). ROS production can be detected and reaches its maximum at approximately 15-20 minutes following treatment (Fig. 1). This response is abolished in the cerk 1-2 (Col-0) and cerk 1-3 (WS-4) mutants (Fig. 1). Intriguingly, despite being derived from a fungal pathogen that has tomato as its sole host, addition of Pichia pastoris-produced Ecp6 protein prevents chitin-triggered ROS production in Arabidopsis wild type ecotypes (Fig 1). This is in accordance with previous results, which showed suppression of ROS generation by Ecp6 upon chitin treatment in tomato ${ }^{21}$. Ecp6 ability to perturb chitin-induced immune responses in tomato is mediated by its LysM2 domain, since P.pastoris-produced Ecp6 mutated in the putative chitin-binding site of LysM2 (Ecp6 ${ }^{\text {T95R }}$ ) no longer suppresses chitin responses ${ }^{20}$. Surprisingly, however, simultaneous application of polymeric chitin and Ecp6 ${ }^{\text {TasR }}$ effectively reduces the generation of ROS in Arabidopsis, similar to wild type Ecp6 protein (Fig. 1). These results suggest that Ecp6 is functional in Arabidopsis but that its mode of action may differ between plant species. Alternatively, since Arabidopsis is less sensitive to chitin than tomato ${ }^{23}$, Ecp6 ability to sequester chitin fragments through its high-affinity binding site may be sufficient to suppress chitin-induced immune responses in Arabidopsis. However, we cannot exclude the possibility that differences between batches of purified protein produced in P. pastoris result in variations of Ecp6 activity irrespective of the plant species tested.

Chitin receptors interact in a ligand-dependent manner to activate downstream signalling ${ }^{24}$. In a previous report, the chitin-triggered interaction between AtCERK1 and AtLYK5 was shown following their production in Arabidopsis protoplasts ${ }^{12}$. To be able to test whether Ecp6 interferes with this complex formation, we intended to confirm the occurrence of an interaction between AtLYK5 and AtCERK1 in transgenic Arabidopsis lines producing $\mathrm{mCitrine-tagged} \mathrm{AtLYK5}$. To this end, we vacuum-infiltrated Arabidopsis leaves with a chitin suspension or water as negative control, and immunopurified AtLYK5mCitrine from crude protein extracts. Immunopurification of AtLYK5-mCitrine following chitin treatment did not result in co-purification of AtCERK1 (Fig. 2). In a further attempt to demonstrate an interaction between AtLYK5 and AtCERK1 using Arabidopsis leaf tissue, we infiltrated the cross-linking agent ethylene glycol bis(succinimidyl succinate) (EGS) 30 
min after chitin treatment. However, we failed to observe co-purification of AtCERK1 with AtLYK5-mCitrine even following cross-linking (Fig. 2). It has been hypothesized that the interaction between AtCERK1 and AtLYK5 is transient upon perception of chitin ${ }^{25}$. Thus, the use of whole Arabidopsis leaves may be inappropriate to visualize this transient interaction. Consequently, based on the results presented here, we were not able to test a possible inhibitory effect on chitin receptor dimerization by Ecp6.

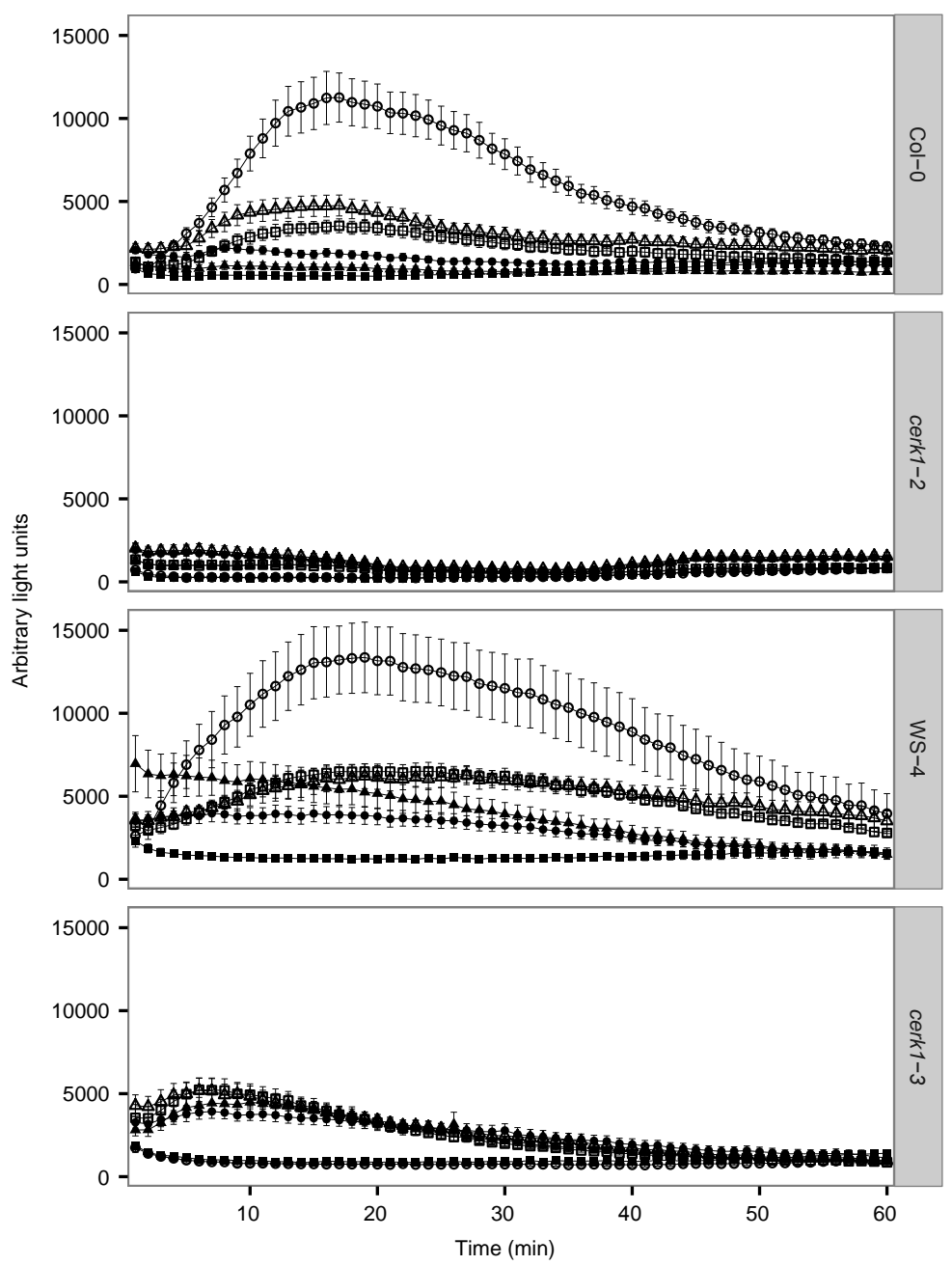

FIGURE 1 | Ecp6 suppresses ROS production in Arabidopsis. Generation of ROS was measured using luminolbased chemiluminescence for 60 min following treatment of Arabidopsis leaf discs with $10 \mu \mathrm{g} / \mathrm{mL}$ polymeric chitin (0), $10 \mu \mathrm{M}$ Ecp6 (O), $10 \mu \mathrm{M} \mathrm{Ecp6}{ }^{\text {T95R }}(\mathbf{\Delta})$, Ecp6+chitin $(\boldsymbol{\Delta})$, Ecp6 ${ }^{\text {T95R }}+$ chitin $(\mathbf{L})$, or water $(\square)$ as negative control. Plotted are chemiluminescence averages of $n=8$ leaf discs \pm S.E.. The figure is representative of at least three independent experiments. 


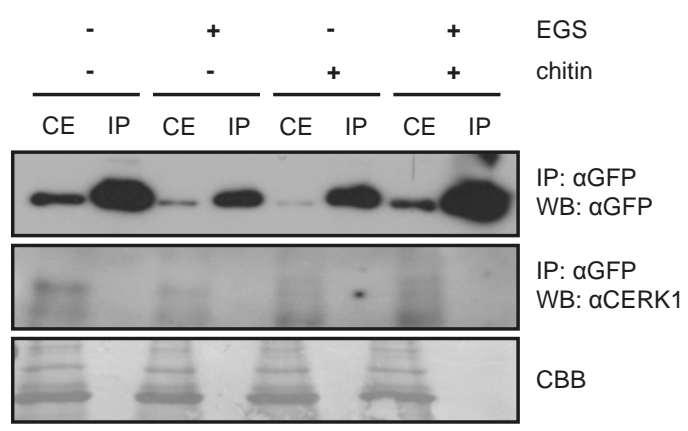

FIGURE 2 | Treatment with a cross-linking agent is not sufficient to demonstrate chitin receptor interaction in stably transformed Arabidopsis lines. Western blot (WB) showing immunopurification (IP) of AtLYK5-mCitrine from crude leaf extracts (CE) of transgenic Arabidopsis treated with water or $10 \mu \mathrm{g} / \mathrm{mL}$ polymeric chitin. Thirty min after elicitation, leaves were treated with the cross-linking agent ethylene glycol bis(succinimidyl succinate) (EGS). Membranes were stained with Coomassie brilliant blue (CBB) to confirm equal loading. The experiment was repeated twice with similar results.

Chitin perception results in the phosphorylation of AtCERK1 and AtLYK5, and AtCERK1 phosphorylation is required for the activation of signal transduction ${ }^{25}$. Phosphorylation can be detected as a band shift in total protein extracts on SDS polyacrylamide gels and serves as a proxy for receptor complex activation. AtCERK1 shifted on gel after chitin treatment in Col-0 and much less in lyk5-2 mutants (Fig. 3a), confirming AtLYK5 involvement. Moreover, immunoprecipitation with chitin beads resulted in the recovery of less AtCERK1 protein from crude extracts following chitin infiltration when compared with water treatment (Fig. 3a). Several receptors, including the leucine-rich repeat (LRR) receptor kinase FLAGELLIN SENSING 2 (FLS2) and the LRR receptor protein Cf4, are internalized and targeted for degradation upon ligand perception ${ }^{26-29}$. Consequently, the overall abundance of FLS2 and Cf4 decreases following treatment with their ligands flg22 and Avr4, respectively ${ }^{27,30,31}$. However, AtCERK1 can be continuously detected at the plasma membrane and no vesicle formation is observed following chitin treatment ${ }^{25}$. Thus, the reduced recovery of AtCERK1 with chitin beads is likely due to the lower availability of chitin-free AtCERK1 molecules following chitin treatment. We then tested whether AtCERK1 phosphorylation is diminished following chitin treatment in the presence of Ecp6. While the lower band for AtCERK1, which represents AtCERK1 in absence of chitintriggered phosphorylation ${ }^{10}$, appeared to be stronger when leaves were treated both with Ecp6 and chitin when compared to treatment with chitin only, AtCERK1 phosphorylation was not suppressed (Fig. 3b). Interestingly, however, immunoprecipitation with chitin beads following total protein extraction resulted in increased recovery of AtCERK1 receptor protein in the presence of Ecp6 and chitin. This suggests that Ecp6 (indirectly) affects AtCERK1 chitin binding. Possibly, Ecp6 sequesters chitin molecules, leaving fewer molecules for AtCERK1, and thus augmenting the amount of AtCERK1 chitin binding sites that remain accessible to immunoprecipitation with chitin beads. Similar results were obtained after co-treatment with chitin and Ecp6 $6^{\text {T95R }}$ (Fig. 3b).

Since Ecp6 interferes with chitin-dependent defense responses in Arabidopsis, but does not markedly affect AtCERK1 phosphorylation, we tested whether Ecp6 can interfere with AtLYK5 internalization. AtLYK5 is internalized into endosomes starting approximately 20 
min and peaking around 60 min after chitin treatment ${ }^{25}$. In addition to polymeric chitin, chitin heptamers (GN7) and octamers (GN8) are able to induce AtLYK5 endocytosis at 10 $\mu \mathrm{M}$ final concentrations (Fig. 4). Surprisingly, although co-infiltration of chitin oligomers with Ecp6 at equimolar concentrations did not change the number of observed vesicles, the vesicles became more prominent (Fig. 4). In contrast, treatment with Ecp6 in the presence of polymeric chitin resulted in the formation of fewer vesicles but did not affect their size (Fig. 4). These observations suggest that Ecp6 affects AtLYK5 internalization in a ligand-dependent manner.

a

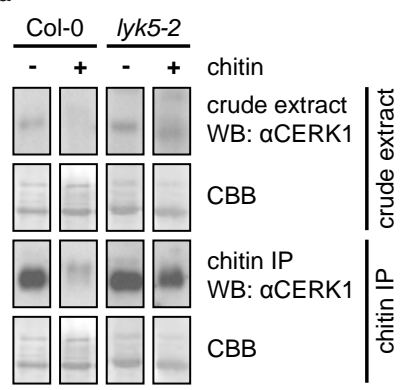

b

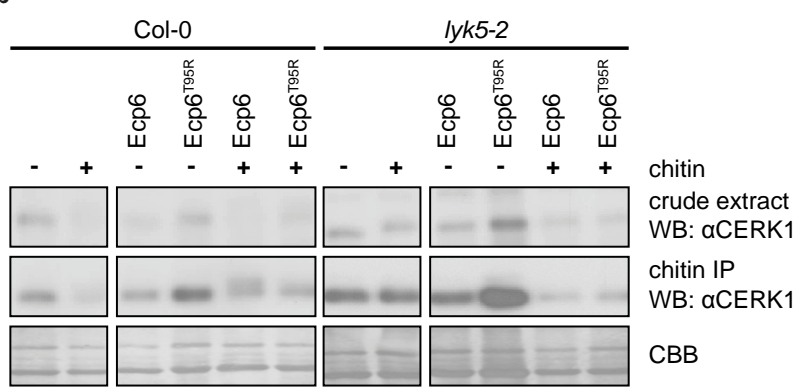

FIGURE 3 | Ecp6 does not prevent AtCERK1 phosphorylation. AtCERK1 was detected using a-CERK1 antibody in crude protein extracts from Col-0 or lyk5-2 leaf tissue after treatment with water or $10 \mu \mathrm{g} / \mathrm{mL}$ polymeric chitin in absence (a) or presence (b) of Ecp6 or Ecp6 $6^{\text {T95R }}(3 \mu \mathrm{M})$. Chitin binding of AtCERK1 was confirmed in pull-downs with chitin beads. Membranes were stained with Coomassie brilliant blue (CBB) after Western blotting to confirm equal loading. The results shown are representative of three independent experiments.

In summary, we show that Ecp6 perturbs chitin-triggered immune responses in Arabidopsis. However, its role and in particular the contribution of the singular LysM2 domain to Arabidopsis chitin receptor inhibition remain elusive. Importantly, Ecp6 is produced by the tomato pathogen C. fulvum and a translation into the tomato system is required to fully unravel Ecp6 functionality.

\section{Materials}

\section{Plant materials}

Plants were grown under short day conditions ( $8 \mathrm{~h}$ photoperiod), at $22^{\circ} \mathrm{C} / 18^{\circ} \mathrm{C}$ during day/night cycles, with $65 \%$ relative humidity and light intensity approximately $150 \mu \mathrm{mol} /$ $\mathrm{m}^{2} \mathrm{~s}$. Plants were used for experiments after 4-6 weeks. In vitro seedlings were grown in liquid $1 / 2$ Murashige and Skoog medium supplemented with $0.5 \%$ sucrose for 2 weeks. The Arabidopsis T-DNA lines used in this study were cerk1-2 (GABI_096F09)7, lyk5-2 (SALK_131911C)7,12 and cerk1-3 (FLAG_GX112, INRA line) ${ }^{32}$.Vesicle formation was observed using transgenic $p L Y K 5:: L Y K 5-m$ Citrine ${ }^{25}$. 


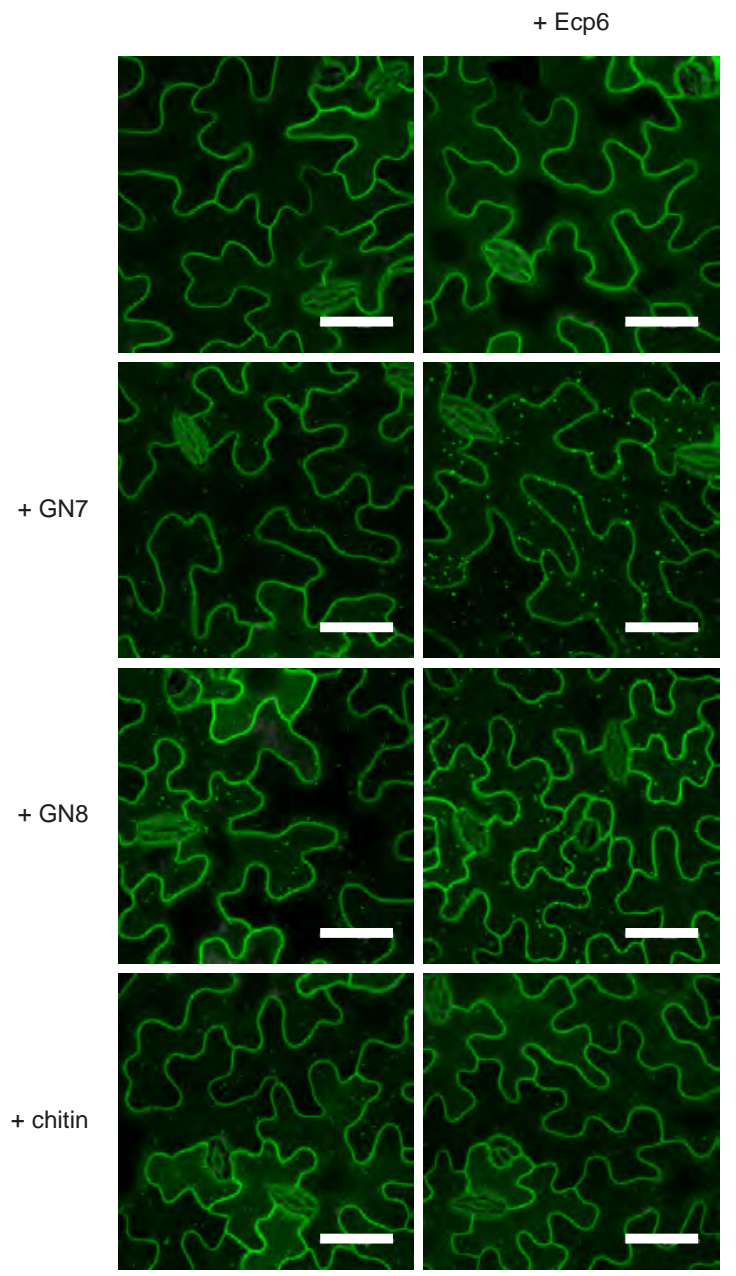

FIGURE 4 | Ecp6 affects chitin-dependent AtLYK5 endocytosis. Leaves of Col-0 plants stably producing AtLYK5-mCitrine were infiltrated with water, $10 \mu \mathrm{M}$ GN7, $10 \mu \mathrm{M}$ $\mathrm{GN} 8$, or $10 \mu \mathrm{g} / \mathrm{mL}$ polymeric chitin with or without $10 \mu \mathrm{M}$ Ecp6. Internalization of AtLYK5-mCitrine was detected 60 min after treatment. Shown are maximum projections of 8 focal planes. Size bar $=30 \mu \mathrm{m}$.

\section{Production and purification of recombinant effector proteins}

Ecp6 was expressed in Pichia pastoris strain GS115 and purified as described previously ${ }^{21,33}$. For treatment of plant tissue, recombinant effector proteins were desalted over PD10 columns (GE Life Sciences) according to manufacturer's instructions.

\section{Chemicals and plant treatments}

For induction of vesicles, chitin (Sigma-Aldrich), and chitooligomers GN7 and GN8 (IsoSep) were vacuum-infiltrated into leaf pieces in absence or presence of $10 \mu \mathrm{M} \mathrm{Ecp6.} \mathrm{Polymeric}$ chitin was used at a concentration of $10 \mu \mathrm{g} / \mathrm{mL}$ and oligomers at $10 \mu \mathrm{M}$. The incubation time was $60 \mathrm{~min}$. For assaying phosphorylation of CERK1 $100 \mu \mathrm{g} / \mathrm{mL}$ chitin was vacuum-infiltrated into detached leaves and incubated for $10 \mathrm{~min}$. To test the suppression of phosphorylation by Ecp6 $10 \mu \mathrm{g} / \mathrm{mL}$ chitin was infiltrated in absence or presence of $3 \mu \mathrm{M}$ Ecp6 or Ecp6 ${ }^{\text {T95R }}$. 


\section{Oxidative burst assay}

Oxidative burst measurements were performed as previously described ${ }^{32}$ with the following modifications: Water was replaced with $100 \mu \mathrm{L}$ of a solution containing 100 $\mu \mathrm{M} \mathrm{L}-012$ substrate and $20 \mu \mathrm{g} / \mathrm{mL}$ horseradish peroxidase. ROS was elicited with $10 \mu \mathrm{g} /$ $\mathrm{mL}$ polymeric chitin in absence or presence of $10 \mu \mathrm{M}$ recombinant effector protein. Eight leaf discs were used for each condition. Luminescence was measured over 60 min using an Infinite ${ }^{\circledR}$ M200 multimode reader (TECAN) in combination with the i.control software package (Version 1.6).

\section{Protein extraction, affinity purification and immunoblotting}

Receptor cross-linking was carried out in vivo by vacuum-infiltration with ethylene glycol bis(succinimidyl succinate) (EGS) of water- or chitin-treated leaves. Total protein extractions and chitin pull-downs were performed as described previously ${ }^{10}$. For receptor co-immunoprecipitations total extracts were mixed with $20 \mu \mathrm{L}$ magnetic chitin beads (New England Biolabs) and incubated at $4^{\circ} \mathrm{C}, 10 \mathrm{rpm}$ for $1 \mathrm{hr}$. Beads were washed three times with $1 \mathrm{~mL}$ TBS-T. Affinity purified proteins were eluted in $40 \mu \mathrm{L} 2 \times$ SDS buffer. Proteins were separated by 8 or $10 \%$ SDS-PAGE and blotted to polyvinylidene difluoride (PVDF) membranes (Millipore). Membranes were probed with anti-CERK1 ${ }^{10,32}$ or HRP-linked antiGFP (ChromoTek). A goat-anti-rabbit alkaline phosphatase conjugate (Sigma-Aldrich) was used as secondary antibody for aCERK1. Reactions were detected using the ImmunStar AP substrate (Bio-Rad) or with SuperSignal West Femto Chemiluminescent Substrate (Thermo Scientific).

\section{Confocal microscopy}

Confocal microscopy was performed on a Leica TCS SP5 system (Leica Microsystems, Wetzlar, Germany) equipped with an argon laser and HyD hybrid detectors. mCitrine was excited at $514 \mathrm{~nm}$, and emission was recorded between 525 and $560 \mathrm{~nm}$. Chlorophyll autofluorescence was detected between 740 and $770 \mathrm{~nm}$. Images were processed using the Leica LAS AF (Version 2.7.2.) software package. All the phenotypes were observed in at least two independent transgenic Arabidopsis lines.

\section{Acknowledgements}

This work was carried out within the framework of the COST action FA1208 Sustain STSM fellowship program in the laboratory of Prof. Dr. Volker Lipka at the Albrecht-von-HallerInstitute, Georg-August-University Göttingen, Germany. 


\section{Author contributions}

H.R. and B.P.H.J.T. designed and conceived the experiments. H.R., E.K.P., J.E. and V.L. performed the experiments and analyzed the data. H.R. and B.H.P.J.T. wrote the manuscript.

\section{References}

1 Kombrink, A., Sánchez-Vallet, A. \& Thomma, B. P. H. J. The role of chitin detection in plant-pathogen interactions. Microb. Infect. 13, 1168-1176, doi:10.1016/j.micinf.2011.07.010 (2011).

2 Antolín-Llovera, M. et al. Knowing your friends and foes - plant receptor-like kinases as initiators of symbiosis or defence. New Phytol. 204, 791-802, doi:10.1111/nph.13117 (2014).

3 Sánchez-Vallet, A., Mesters, J. R. \& Thomma, B. P. H. J. The battle for chitin recognition in plantmicrobe interactions. FEMS Microbiol. Rev. 39, 171 183, doi:10.1093/femsre/fuu003 (2015).

$4 \mathrm{Kaku}, \mathrm{H}$. et al. Plant cells recognize chitin fragments for defense signaling through a plasma membrane receptor. Proc. Natl. Acad. Sci. USA 103, 11086-11091, doi:10.1073/pnas.0508882103 (2006).

5 Shimizu, T. et al. Two LysM receptor molecules, CEBiP and OsCERK1, cooperatively regulate chitin elicitor signaling in rice. Plant J. 64, 204-214, doi:10.1111/j.1365-313X.2010.04324.x (2010).

6 Hayafune, M. et al. Chitin-induced activation of immune signaling by the rice receptor CEBiP relies on a unique sandwich-type dimerization. Proc. Natl. Acad. Sci. USA 111, E404-413, doi:10.1073/ pnas.1312099111 (2014).

7 Miya, A. et al. CERK1, a LysM receptor kinase, is essential for chitin elicitor signaling in Arabidopsis. Proc. Natl. Acad. Sci. USA 104, 1961319618, doi:10.1073/pnas.0705147104 (2007).

8 Wan, J. R. et al. A LysM receptor-like kinase plays a critical role in chitin signaling and fungal resistance in Arabidopsis. Plant Cell 20, 471-481, doi:10.1105/tpc.107.056754 (2008).

9 lizasa, E., Mitsutomi, M. \& Nagano, Y. Direct binding of a plant LysM receptor-like kinase, LysM RLK1/CERK1, to chitin in vitro. J. Biol. Chem. 285 doi:10.1074/Jbc.M109.027540 (2010).
10 Petutschnig, E. K., Jones, A. M., Serazetdinova, L., Lipka, U. \& Lipka, V. The lysin motif receptor-like kinase (LysM-RLK) CERK1 is a major chitin-binding protein in Arabidopsis thaliana and subject to chitin-induced phosphorylation. J. Biol. Chem. 285, 28902-28911, doi:10.1074/jbc.M110.116657 (2010).

11 Liu, T. T. et al. Chitin-induced dimerization activates a plant immune receptor. Science $\mathbf{3 3 6}$, 1160-1164, doi:10.1126/science.1218867 (2012).

12 Cao, Y. et al. The kinase LYK5 is a major chitin receptor in Arabidopsis and forms a chitininduced complex with related kinase CERK1. Elife, doi:10.7554/eLife.03766 (2014).

13 Shinya, T. et al. Functional characterization of CEBiP and CERK1 homologs in Arabidopsis and rice reveals the presence of different chitin receptor systems in plants. Plant Cell Physiol. 53, 1696-1706, doi:10.1093/pcp/pcs113 (2012).

14 Faulkner, C. et al. LYM2-dependent chitin perception limits molecular flux via plasmodesmata. Proc. Natl. Acad. Sci. USA 110, 9166-9170, doi:10.1073/pnas.1203458110 (2013).

15 Marshall, R. et al. Analysis of two in planta expressed LysM effector homologs from the fungus Mycosphaerella graminicola reveals novel functional properties and varying contributions to virulence on wheat. Plant Physiol. 156, 756-769, doi:10.1104/pp.111.176347 (2011).

16 Mentlak, T. A. et al. Effector-mediated suppression of chitin-triggered immunity by Magnaporthe oryzae is necessary for rice blast disease. Plant Cell 24, 322-335, doi:10.1105/tpc.111.092957 (2012).

17 Takahara, $\mathrm{H}$. et al. Colletotrichum higginsianum extracellular LysM proteins play dual roles in appressorial function and suppression of chitin-triggered plant immunity. New Phytol., doi:10.1111/nph.13994 (2016). 
18 van den Burg, H. A., Harrison, S. J., Joosten, M. H. A. J., Vervoort, J. \& de Wit, P. J. G. M. Cladosporium fulvum Avr4 protects fungal cell walls against hydrolysis by plant chitinases accumulating during infection. Mol. Plant-Microbe Interact. 19, 1420-1430, doi:10.1094/MPMI-19-1420 (2006).

19 van Esse, H. P., Bolton, M. D., Stergiopoulos, I., de Wit, P. J. G. M. \& Thomma, B. P. H. J. The chitinbinding Cladosporium fulvum effector protein Avr4 is a virulence factor. Mol. Plant-Microbe Interact. 20, 1092-1101, doi:10.1094/MPMI-20-91092 (2007).

20 Sánchez-Vallet, A. et al. Fungal effector Ecp6 outcompetes host immune receptor for chitin binding through intrachain LysM dimerization. Elife, doi:10.7554/eLife.00790 (2013).

21 de Jonge, R. et al. Conserved fungal LysM effector Ecp6 prevents chitin-triggered immunity in plants. Science 329, 953-955, doi:10.1126/ science.1190859 (2010).

22 Kombrink, A. \& Thomma, B. P. H. J. LysM Effectors: Secreted proteins supporting fungal life. PLOS Pathog. 9, doi:10.1371/journal.ppat.1003769 (2013).

23 Felix, G., Regenass, M. \& Boller, T. Specific perception of subnanomolar concentrations of chitin fragments by tomato cells - induction of extracellular alkalinization, changes in protein-phosphorylation, and establishment of a refractory state. Plant J. 4, 307-316, doi:DOI 10.1046/j.1365-313X.1993.04020307.x (1993).

24 Shinya, T., Nakagawa, T., Kaku, H. \& Shibuya, N. Chitin-mediated plant-fungal interactions: catching, hiding and handshaking. Curr. Opin. Plant Biol. 26, 64-71, doi:10.1016/j.pbi.2015.05.032 (2015).

25 Erwig, J., Ghareeb, H., Kopischke, M., Hacke, R., Matei, A., Petutschnig, E.K., Lipka, V. Chitininduced and CHITIN ELICITOR RECEPTOR KINASE1 (CERK1) phosphorylation-dependent endocytosis of Arabidopsis thaliana LYSIN MOTIF-CONTAINING RECEPTOR-LIKE KINASE5 (LYK5). New Phytol. 215,
382-396, doi:10.1111/nph.14592 (2017).

26 Beck, M., Zhou, J., Faulkner, C., MacLean, D. \& Robatzek, S. Spatio-temporal cellular dynamics of the Arabidopsis flagellin receptor reveal activation status-dependent endosomal sorting. Plant Cell 24, 4205-4219, doi:10.1105/tpc.112.100263 (2012).

27 Postma, J. et al. Avr4 promotes Cf-4 receptorlike protein association with the BAK1/ SERK3 receptor-like kinase to initiate receptor endocytosis and plant immunity. New Phytol. 210, 627-642, doi:10.1111/nph.13802 (2016).

28 Robatzek, S., Chinchilla, D. \& Boller, T. Ligandinduced endocytosis of the pattern recognition receptor FLS2 in Arabidopsis. Genes Dev. 20, 537542, doi:10.1101/gad.366506 (2006).

29 Spallek, T. et al. ESCRT-I mediates FLS2 endosomal sorting and plant immunity. PLoS Genet. 9, e1004035, doi:10.1371/journal.pgen.1004035 (2013).

$30 \mathrm{Lu}, \mathrm{D}$. et al. Direct ubiquitination of pattern recognition receptor FLS2 attenuates plant innate immunity. Science 332, 1439-1442, doi:10.1126/ science.1204903 (2011).

31 Smith, J. M., Salamango, D. J., Leslie, M. E., Collins, C. A. \& Heese, A. Sensitivity to Flg22 is modulated by ligand-induced degradation and de novo synthesis of the endogenous flagellin-receptor FLAGELLIN-SENSING2. Plant Physiol. 164, 440-454, doi:10.1104/pp.113.229179 (2014).

32 Gimenez-lbanez, S. et al. AvrPtoB targets the LysM receptor kinase CERK1 to promote bacterial virulence on plants. Curr. Biol. 19, 423-429, doi:10.1016/j.cub.2009.01.054 (2009).

33 van den Burg, H. A., de Wit, P. J. \& Vervoort, J. Efficient $13 \mathrm{C} / 15 \mathrm{~N}$ double labeling of the avirulence protein AVR4 in a methanol-utilizing strain (Mut+) of Pichia pastoris. J. Biomol. NMR 20, 251-261 (2001). 



\title{
Chapter 4
}

\section{A Lys $M$ receptor kinase mediates chitin-triggered defense responses in tomato}

\author{
Hanna Rovenich', Elena K. Petutschnig ${ }^{2}$, Oliver Valerius ${ }^{3}$, \\ Malaika K. Ebert', Grardy C.M. van den Berg', Bart P.H.J. Thomma'
}

'Laboratory of Phytopathology, Wageningen University, 6708 PB Wageningen, The Netherlands; ${ }^{2}$ Department of Plant Cell Biology, Schwann-Schleiden Centre, Georg-August-University Göttingen, 37077 Göttingen, Germany;

${ }^{3}$ Department of Molecular Microbiology and Genetics, Institute of Microbiology and Genetics, Georg-August-University Göttingen, 37077 Göttingen, Germany 


\begin{abstract}
Plants detect the presence of potential fungal pathogens by sensing the conserved cell wall component chitin. To date, all plant chitin receptors identified belong to the class of membrane-exposed lysin motif (LysM)-containing receptor proteins. Here, we identify two chitin-binding LysM receptors of tomato using an affinity purification approach. Based on their phylogenetic relationship to well-characterized chitin receptors of rice and Arabidopsis thaliana, the tomato receptor candidates were named SICEBiP and SILYK4, respectively. Silencing of SILYK4, but not of SICEBiP, resulted in compromised tomato responsiveness to chitin. These results suggest that SILYK4 is a major component of the receptor complex in tomato that activates the canonical chitin signal transduction pathway.
\end{abstract}




\section{Introduction}

Plants detect the presence of invading microbes through cell surface-localized receptor molecules, which recognize either microbe-derived or modified-self molecules that indicate invasion'. This recognition leads to a series of cellular events that either promote or restrict microbial colonization ${ }^{2}$. The activation of immune responses, which include the generation of extracellular reactive oxygen species (ROS), an increase in cytosolic calcium concentrations, the activation of mitogen-activated protein (MAP) kinase and calciumdependent protein kinase (CDPK) cascades as well as changes in gene expression, protect the plant from invasion by potential pathogens ${ }^{3}$.

Bacterial molecules that are recognized by plant receptor proteins include flagellin, elongation factor Tu (EF-Tu), peptidoglycan or lipopolysaccharide 3 . In contrast, filamentous microbes are generally perceived due to the presence of their major cell wall components chitin or $\beta$-glucan ${ }^{4,5}$. Chitin consists of $\beta$-1,4-linked polymers of $N$-acetylglucosamine (GlcNAc) with varying degrees of polymerization. While longer chitin oligomers are potent inducers of immune responses ${ }^{6,7}$, chitin tetra- and heptamers have been implicated in mutualistic symbiosis ${ }^{8,9}$. Interestingly, bacterial peptidoglycan as well as lipo-chitooligosaccharides (LCOs) produced by beneficial rhizobacteria and arbuscular mycorrhizal (AM) fungi, respectively, are chitin derivatives.

Until now, the receptor molecules that have been implicated in the recognition of GICNAccontaining molecules belong to the group of lysin motif (LysM) receptor proteins, which are classified as receptor kinases (RKs) or receptor proteins (RPs) ${ }^{10}$. The extracellular portion of LysM-RKs, which contains the LysM domains, is coupled to an intracellular kinase domain via a single pass transmembrane domain. Based on differences in their kinase domain features, LysM-RKs are further divided into LYKs and LYRs ${ }^{10,11}$. In contrast to LYKs, the kinase domains of LYRs lack most structural components required for kinase activity. LysM-RPs (also referred to as LYPs) are membrane-bound via a glycosyl phosphatidylinositol (GPI) anchor and lack an intracellular kinase domain for activation of downstream signaling.

Signal transduction requires the presence of receptor complexes containing LYK receptor molecules, which relay the signal via their intracellular kinase domain. For example, in Arabidopsis thaliana (hereafter referred to as Arabidopsis), the LYR AtLYK5 binds chitin with high affinity and forms a heteromeric complex with the receptor kinase CERK1 to initiate chitin signalling ${ }^{12}$. While AtLYK5 has been proposed as the main chitin receptor in Arabidopsis, its paralogue AtLYK4 has also been implicated in chitin recognition and only lyk4lyk5 double mutants show a complete loss of chitin responsiveness ${ }^{12-14}$. As in Arabidopsis, rice (Oryza sativa) CERK1 is required for chitin signaling following its ligand-induced association with the LYP receptor OsCEBiP ${ }^{15-18}$. Interestingly, one of the Arabidopsis CEBiP orthologues, AtLYM2 controls the chitin-induced flux across plasmodesmata and resistance to fungal 
pathogens independent of AtCERK $1^{19,20}$. In addition to chitin signaling, CERK1 from both rice and Arabidopsis has been shown to be required for peptidoglycan-triggered immunity ${ }^{21-24}$. Interestingly, in addition to its role in immunity, OsCERK1 has also been implicated in AM symbiosis that is established upon perception of fungal $\mathrm{LCO}^{18,25}$. These findings suggest that CERK1 likely acts as a co-receptor in different receptor complexes ${ }^{4}$. Similar to fungal LCOs, also bacterial LCOs are recognized by LysM receptor pairs in leguminous plant species during the establishment of symbiosis with rhizobacteria ${ }^{26-29}$.

Based on the findings in rice and Arabidopsis, it is tempting to assume that also in tomato LysM receptors are required for the perception of GlcNAc-containing molecules. Indeed, the LYR receptor kinase SILYK10 has recently been shown to play a role in colonization by the AM fungus Rhizophagus irregularis ${ }^{30}$. Indirect evidence suggests that the CERK1 orthologues SILYK1 and SILYK13 may be required for tomato immunity to bacterial pathogens as they are targeted by the bacterial effector protein AvrPtoB to suppress plant immune responses ${ }^{31}$.

Here we identified two LysM receptors, SILYK4 and SICEBiP, with a putative role in chitin perception using a biochemical approach. Virus-induced gene silencing of SILYK4, but not of SICEBiP, resulted in reduced chitin responsiveness, suggesting that SILYK4 mediates chitintriggered immune responses in tomato.

\section{Results}

\section{Mass spectrometry identifies two LysM receptors as candidate chitin receptors}

To identify the receptor protein(s) involved in chitin perception in tomato, we isolated microsomal proteins from tomato cv. Heinz 1706 leaf tissue for affinity purification with magnetic chitin beads ${ }^{32}$. Specifically bound proteins were eluted either with chitohexaose ((GlcNAc) 6 abbreviated as GN6) or chitosan. In order to elute the remaining non-specifically bound proteins, chitin beads were boiled in sample buffer. Following size-separation by SDS-PAGE, proteins in the range of 50-100 kDa were subjected to trypsin digestion. Subsequent mass spectrometric analysis yielded several unique peptides in both GN6 and chitosan eluents for only two LysM-containing receptors encoded by Solyc02g089900 and Solyc01g112080 (Table 1; Supplementary Fig. 1). Unique peptides for the same receptors were also detected in the on-bead fractions, suggesting that the elution with GN6 and chitosan had been incomplete. Moreover, only 0 and 1 unique peptide were found in the supernatant following affinity purification (unbound fraction) for Solyc02g089900 and Solyc01g112080, respectively, demonstrating that the majority of both proteins present in the whole leaf extracts bound to chitin beads (Table 1). 
TABLE 1 | Identification of chitin-binding LysM proteins of tomato (Solanum lycopersicum Heinz 1706) Chitin-binding proteins identified from microsomal fractions. Numbers represent unique peptides identified by Sequest and/or Mascot analyses.

\begin{tabular}{|c|c|c|c|c|c|c|c|c|c|c|c|}
\hline Gene ID & Name & 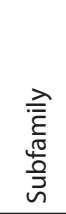 & 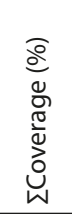 & $\begin{array}{l}\text { I } \\
\text { I } \\
\stackrel{N}{N}\end{array}$ & 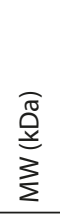 & $\overline{2}$ & $\begin{array}{l}0 \\
\frac{0}{5} \\
0 \\
\frac{0}{5} \\
5\end{array}$ & $\begin{array}{l}\frac{\vec{c}}{\Delta} \\
\stackrel{\vec{J}}{U} \\
z^{\circ}\end{array}$ & 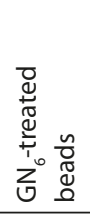 & 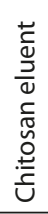 & 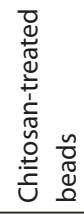 \\
\hline Solyc02g089900 & SILYK4 & LYR & 17.2 & 645 & 70 & 5.3 & 0 & 3 & 4 & 4 & 4 \\
\hline Solyc01g112080 & SICEBiP & LYP & 18 & 345 & 37 & 5.4 & 1 & 4 & 4 & 3 & 3 \\
\hline
\end{tabular}

Solyc02g089900 codes for a membrane-bound LYR with three surface-exposed LysM domains that is closely related to Arabidopsis LYK4, and is therefore referred to as SILYK4 (Fig. 1; Fig. 2) ${ }^{30}$. Phylogenetic analysis of full-length LysM receptor protein sequences showed that, in addition to AtLYK4, SILYK4 forms a clade together with AtLYK5, SILYK6, SILYK7, and SILYK15 on a longer branch, as was shown previously ${ }^{30}$. Solyc01g112080 encodes a receptor protein with extracellular LysM domains that are membrane-bound via a GPI anchor (Fig. 2). Sequence analysis with the domain prediction algorithm InterPro (http://www.ebi.ac.uk/interpro/) suggested the presence of two LysM domains in SICEBiP. However, amino acid sequence alignment with its orthologue OsCEBiP suggests that SICEBiP, like OsCEBiP, Medicago truncatula LYM2 and Arabidopsis LYM2, contains three instead of two LysMs (Fig. 1; Fig. 2; Supplementary Fig. 2) ${ }^{16,33-35}$. Considering the role of their orthologues in chitin signaling in Arabidopsis, rice and M. truncatula, we further investigated the role of SILYK4 and SICEBiP in chitin recognition of tomato.

\section{SILYK4 and SICEBiP gene expression is induced upon fungal infection}

To analyze the expression patterns of the two putative tomato chitin receptor genes, we first examined publicly available transcriptome data (see Methods section for details). The expression levels of both SICEBiP and SILYK4 varied greatly between the two tomato cultivars analyzed (Heinz 1706 and Moneymaker) as well as among the various tissues tested (Supplementary Fig. 2). SILYK4 is mainly expressed in roots in tomato cv. Moneymaker, whereas transcript accumulation is the highest in mature fruits of tomato cv. Heinz (Supplementary Fig. 2a). In contrast, SICEBiP is highly expressed in root tissue of both cultivars (Supplementary Fig. 2b) ${ }^{30}$. Moreover, in tomato cv. Moneymaker expression values vary little between root, stem and leaf tissue, whereas few SICEBiP transcripts were detected in leaves, flowers and mature fruits of tomato cv. Heinz 1706. 


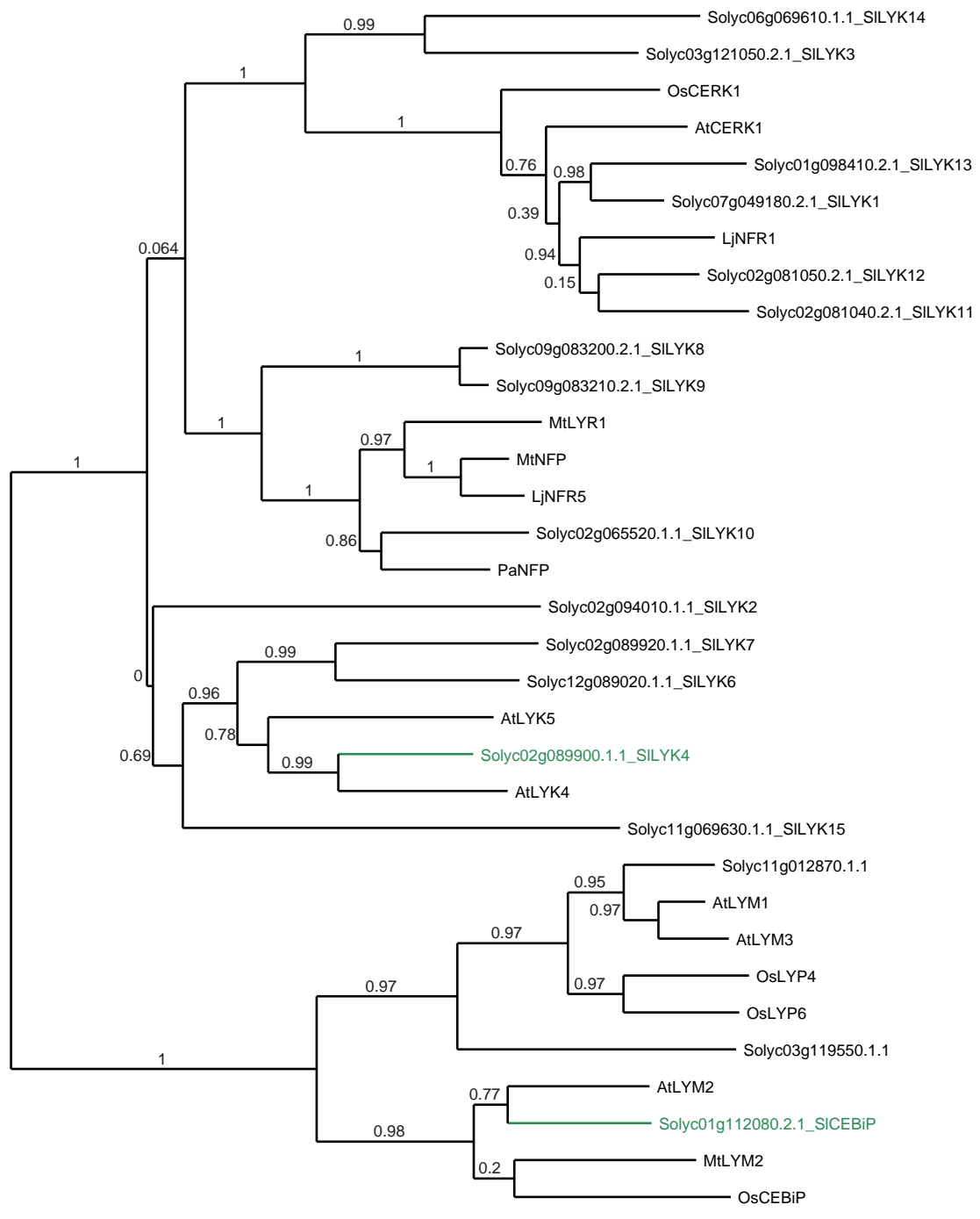

0.7

FIGURE 1 | Phylogenetic analysis of LysM receptor proteins. Tomato LysM proteins were selected based on the presence of an extracellular domain containing LysM motifs as well as their predicted localization to the plasma membrane due to the presence of a predicted transmembrane domain or a GPI anchor. Their phylogenetic relationship to LysM receptor proteins of rice, Arabidopsis, Lotus japonicas, and Medicago truncatula was inferred based on the maximum likelihood method. SILYK4 and SICEBiP are highlighted in green. 


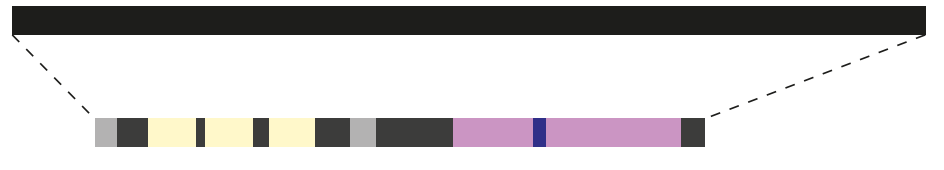

SILYK4 (646 aa)

100 aa

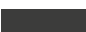

SICEBiP $(1,722$ bp $)$

SICEBiP (346 aa)

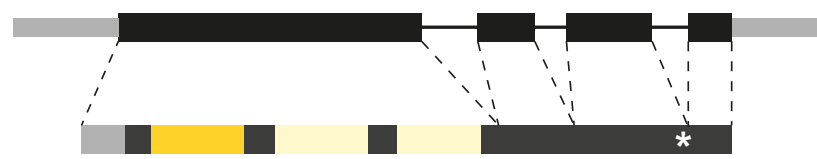

$200 \mathrm{bp}$

50 aa

FIGURE 2 | Graphic representation of gene and encoded protein structures of putative tomato chitin receptors. Genes are represented by black boxes (exons), black lines (introns) and grey boxes ( $5^{\prime}$ and $3^{\prime}$ UTR). The protein structures include signal peptides and the transmembrane domain for SILYK4 (grey boxes), LysM domains predicted by InterPro (yellow), one LysM domain in SICEBiP inferred from pairwise alignment with OsCEBiP (Supplementary Fig. 2) (orange box), and the intracellular kinase domain with the catalytic loop of SILYK4 (violet and blue, respectively). The white asterisk marks the predicted site for the attachment of a GPI anchor in SICEBiP.

Next, changes in SILYK4 and SICEBiP gene expression upon challenge with a fungal pathogen were tested. To this end, tomato plants were inoculated with the vascular wilt fungus Verticillium dahliae and stem tissue was collected at 4, 8, and 12 days following inoculation. Quantitative RT-PCR analysis showed that transcript accumulation of both genes is enhanced during pathogen infection compared to healthy control plants (Fig. 3). SICEBiP expression levels peak at around 8 days post inoculation (dpi), whereas SILYK4 expression is highest at $4 \mathrm{dpi}$ and then gradually decreases. These results suggest that both SICEBiP and SILYK4 may be implicated in chitin perception during plant invasion by fungal pathogens.

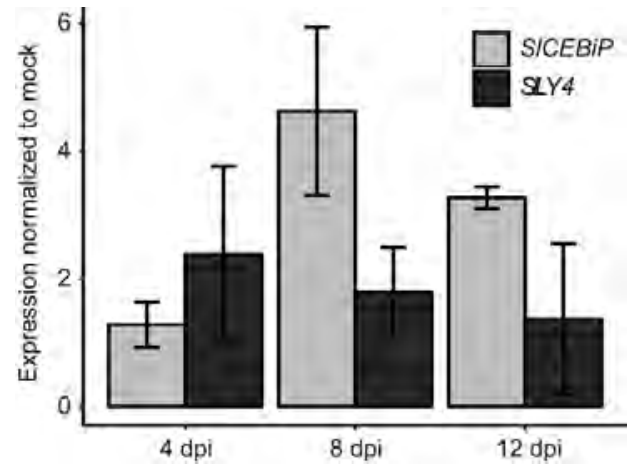

FIGURE 3 | SILYK4 and SICEBiP are expressed in tomato stem tissue during Verticillium infection. Tomato stem tissue was harvested at 4, 8, and 12 days following inoculation with $V$. dahliae. LysM receptor gene expression levels were determined by qRT-PCR with gene-specific primers and primers targeting the tomato tubulin gene for calibration (Supplementary Table 1). Shown are expression values relative to mock-treated tissue of three biological replicates consisting of pools of three plants from a single experiment (mean \pm S.E.). This experiment was performed twice.

\section{Silencing of SILYK4 impairs chitin-triggered responses}

When perceived by plants, chitin triggers the activation of multiple downstream responses, including the generation of reactive oxygen species (ROS) and changes in gene expression. In order to determine whether SILYK4 or SICEBiP contributes to chitin recognition and, 
thus, the activation of chitin-triggered immunity in tomato, we generated tobacco rattle virus (TRV)-based constructs to silence both genes separately. Tomato plants were treated with TRV:SILYK4, TRV:SICEBiP or TRV:GUS as negative control. Three weeks later, two of the youngest, fully expanded leaves were collected from each plant and tested for their ability to respond to chitin. Silencing efficiency and specificity were confirmed by quantitative RT-PCR in mock (water)-treated samples (Supplementary Fig. 5). As expected, treatment with GN6 resulted in ROS generation in GUS-silenced plants within 2.5 min (Fig. 4a). However, silencing of SILYK4 greatly impaired the oxidative burst, whereas the reduction in SICEBiP transcripts had little to no effect (Fig. 4a). To confirm these results, we tested the induction of the three chitin responsive genes SIBAP2, SIERF5, and SICAL-like in mock- and GN6-treated leaf discs of TRV-treated plants ${ }^{36,37}$. Preliminary results show that similar to the oxidative burst, chitin-induced gene expression is impaired in SILYK4-silenced plants compared to the TRV:GUS controls (Fig. 4b-d). These findings suggest that SILYK4, but not SICEBiP, plays a role in tomato chitin recognition.
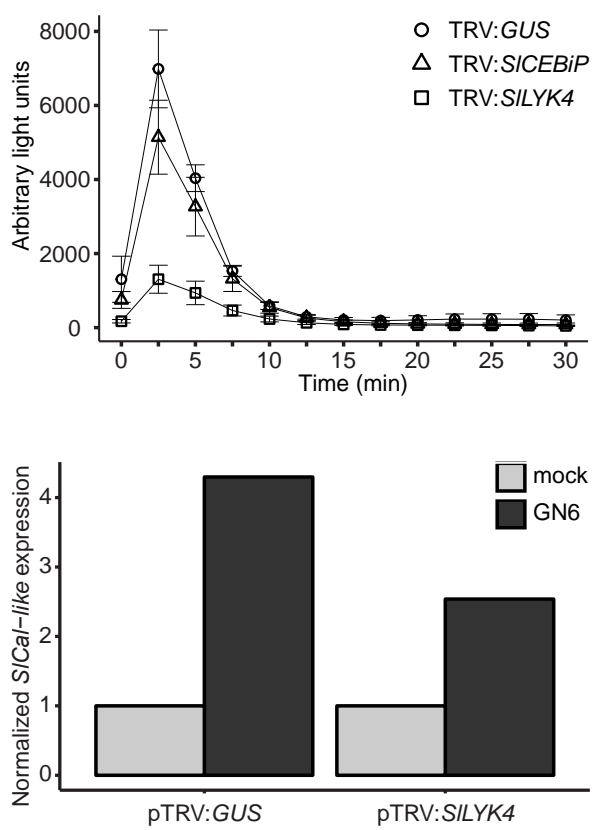

b

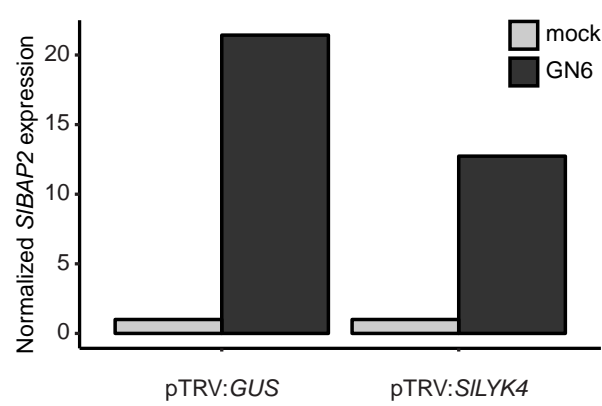

d

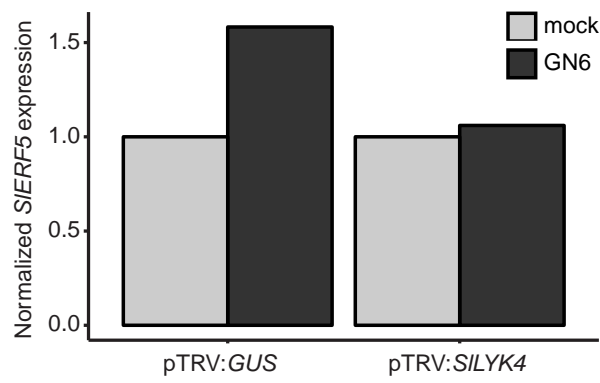

FIGURE 4 | Silencing of SILYK4 results in a reduction of chitin responsiveness in tomato. a, Generation of ROS was measured in leaf discs of ten TRV-treated tomato plants for 30 min following elicitation with $10 \mu \mathrm{M}$ chitohexamer (GN6) using a luminol-based chemiluminescencec assay. Plotted are chemiluminescence averages of $n=80$ leaf discs \pm S.E. normalized to water treatments. The graph is representative of three independent experiments. b-d, Eight leaf disks of three TRV-treated tomato plants were treated either with $10 \mu \mathrm{M}$ GN6 or water as negative control. One hour after treatment the samples were pooled for each treatment. GN6-triggered changes in the expression of SIBAP2 (b), SIERF5 (c) and SICAL-like (d) (encoding homologs of chitin-responsive Arabidopsis genes ${ }^{36}$ ) were determined by qRT-PCR. The bars display normalized transcript levels of chitinresponsive genes relative to the constitutively expressed tomato tubulin gene. 


\section{Discussion}

LysM receptor proteins mediate recognition of a broad range of microbe-derived structural patterns, including bacterial peptidoglycan, fungal chitin and derivatives thereof ${ }^{2,4,38}$. The perception of such patterns triggers plant responses that either lead to the establishment of a mutually beneficial plant-microbe interaction or to the activation of plant immunity that restricts microbial colonization². Here we identified two chitinbinding LysM receptors from tomato and investigated their role in the activation of chitintriggered immune responses.

Affinity purification with chitin beads resulted in the recovery of the two receptor candidates SICEBiP and SILYK4 (Table 1). Surprisingly, both candidates eluted following treatment with GN6 and with chitosan. In contrast to chitin, chitosan oligomers have previously been reported to be only weak immunity elicitors and weak competitors of radiolabeled chitopentaose in tomato ${ }^{39}$. However, the role of chitosan as an inducer of immune responses remains controversial, as it has been reported to have strong effects on other plant species ${ }^{40,41}$. Due to the structural relatedness of chitin and chitosan, we cannot exclude that tomato chitin receptors have some affinity for chitosan, which resulted in the (partial) elution of SILYK4 and SICEBiP from chitin beads.

Despite its ability to bind chitin, silencing of SICEBiP did not result in a reduction of chitintriggered generation of ROS (Fig. 4a). This suggests that SICEBiP is not part of the chitin receptor complex that activates the canonical chitin signal transduction pathway in tomato. Similar to SICEBiP, its closest homolog AtLYM2 was identified based on its chitinbinding activity ${ }^{32,42}$. However, lym2 mutants were not affected in AtCERK1-mediated chitin responses suggesting that AtLYM2 functions in a CERK1-independent pathway ${ }^{19}$. Indeed, AtLYM2 regulates chitin-triggered plasmodesmatal fluxes, which are required for resistance to the fungal pathogen Botrytis cinerea ${ }^{19}$. Thus, it is possible that also SICEBiP has a more specialized role in chitin perception.

In contrast to SICEBiP, SILYK4 is required for the chitin-triggered generation of ROS (Fig. 4a). Additionally, silencing of SILYK4 impaired the induction of chitin-responsive genes (Fig. 4b-d). However, this experiment was carried out only once and requires repetition. Phylogenetic analysis showed that SILYK4 is closely related to the Arabidopsis receptor kinases AtLYK4 and AtLYK5 (Fig. 1) ${ }^{30}$. Like its Arabidopsis orthologues, SILYK4 belongs to the group of LYR receptor kinases that lack a glycine-rich loop and display a degenerate HRD motif in their catalytic loop, rendering their kinase domains inactive ${ }^{11,12,30}$. Since its kinase lacks the important structural features for functionality, it is likely that SILYK4 associates with a co-receptor upon ligand perception which is reminiscent of other LYR receptors ${ }^{2}$. However, we did not identify a LysM receptor protein with an active kinase domain following affinity purification with chitin beads suggesting that this co-receptor may not directly be involved in ligand binding. In rice, chitin binding is mediated by 
OsCEBiP ${ }^{17,43}$, whereas OsCERK1 is recruited into the receptor complex following sandwichlike dimerization of two OsCEBiP molecules around the chitin ligand ${ }^{16}$. When challenged with fungal pathogens, tomato plants silenced for the closest CERK1 homolog SILYK1 did not display marked differences in resistance compared to control plants (A. Kombrink, personal communication). This indicates that SILYK1 is not part of the chitin receptor complex. However, we cannot rule out that gene silencing was insufficient to result in reduced resistance to fungal pathogens. Thus, the identification of additional components of the chitin receptor complex in tomato may require transgenic knockout tomato lines that can be assessed for their ability to mount immunity in response to fungal invasion.

\section{Methods}

\section{Receptor candidate purification}

Microsomal fractions were prepared from tomato (Solanum lycopersicum) cv. Heinz 1706 leaf tissue and used for chitin or chitosan affinity enrichment as described previously ${ }^{32}$. Proteins from unbound, eluent and bead fractions were separated by SDS-PAGE. Gel slices containing proteins of 50-100 kDa were utilized for tryptic digestion and purification for LC-MS/MS.

\section{Candidate identification by liquid chromatography-mass spectrometry}

LC-MS analysis was performed with an Orbitrap Velos Pro ${ }^{\mathrm{TM}}$ Hybrid Ion Trap-Orbitrap mass spectrometer. 1-5 $\mu \mathrm{l}$ of peptide solutions were loaded and washed on an Acclaim PepMAP 100 pre-column (\#164564, $100 \mu \mathrm{m} \times 2 \mathrm{~cm}, \mathrm{C18}, 3 \mu \mathrm{m}, 100 \AA$, Thermo Fisher Scientific) with $100 \%$ loading solvent $\mathrm{A}\left(98 \% \mathrm{H}_{2} \mathrm{O}, 2 \%\right.$ acetonitrile, $\left.0.07 \% \mathrm{TFA}\right)$ at a flow rate of $25 \mu \mathrm{l} / \mathrm{min}$ for $6 \mathrm{~min}$. Peptides were separated by reverse phase chromatography on an Acclaim PepMAP RSLC column (\#164540, $75 \mu \mathrm{m} \times 50 \mathrm{~cm}, \mathrm{C18}, 3 \mu \mathrm{m}, 100 \AA$, Thermo Fisher Scientific) with a gradient from $98 \%$ solvent $\mathrm{A}\left(\mathrm{H}_{2} \mathrm{O}, 0.1 \%\right.$ formic acid) and $2 \%$ solvent $B\left(80 \%\right.$ acetonitrile, $20 \% \mathrm{H}_{2} \mathrm{O}, 0.1 \%$ formic acid) to $42 \%$ solvent $B$ for $95 \mathrm{~min}$ and to $65 \%$ solvent $B$ for the following $26 \mathrm{~min}$ at a flow rate of $300 \mathrm{nl} / \mathrm{min}$. Peptides eluting from the chromatographic column were on-line ionized by nanoelectrospray at $2.4 \mathrm{kV}$ with the Nanospray Flex lon Source (Thermo Fisher Scientific). Full scans of the ionized peptides were recorded within the Orbitrap FT analyzer of the mass spectrometer within a mass range of $300-1850 \mathrm{~m} / \mathrm{z}$ at a resolution of 30.000 . Collision-induced dissociation fragmentation of data-dependent top-fifteen peptides was performed with the LTQ Velos Pro linear ion trap. Data acquisition and programming were carried out with the XCalibur 2.2 software (Thermo Fisher Scientific). A UniProt-derived Solanum lycopersicum-specific database (http://www.uniprot.org, Proteome ID UP000004994, 33952 entries) was used 
for database searches with SequestHT and Mascot search engines. Proteins were identified with the Proteome Discoverer ${ }^{\mathrm{TM}} 1.4$ software. The digestion mode was set to trypsin and the maximum of missed cleavage sites to two. Carbamidomethylation of cysteins was set as fixed modification, oxidation of methionines, and biotinylation of lysines were set as variable modifications (if required). The mass tolerance was 10 ppm for precursor ions and $0.6 \mathrm{Da}$ for fragment ions. The decoy mode was revert with a false discovery rate of 0.01 .

\section{Phylogeny of LysM receptors and protein sequence analyses}

Selected sequences of LysM domain-containing protein sequences were retrieved from the solgenomics network (http://solgenomics.net) for tomato (ITAG3.0), from TAIR (http://arabidopsis.org) for Arabidopsis, from Phytozome 12 (http://phytozome.jgi.doe. gov) for rice (v7) and Medicago truncatula (Mt4.0v1), and UniProt (http://uniprot.org) for Lotus japonicus. The sequences were loaded into the "one click" phylogeny.fr server for phylogenetic analysis, ignoring alignment curation by Gblocks ${ }^{44}$.

The sequences of SILYK4 and SICEBiP were further analysed with SignaIP 4.1 and TargetP 1.1 (http://www.cbs.dtu.dk/services/) to predict the presence of signal peptides and extracellular localization of both candidates. The localization of the LysM domains in the extracellular region of SILYK4 was adopted from a previous study ${ }^{30}$, whereas the LysM domains of SICEBiP were predicted with InterPro (http://www.ebi.ac.uk/interpro/). The GPI modification site in SICEBiP was predicted with the big-PI plant server (http://mendel. imp.ac.at/gpi/plant_server.html).

\section{Candidate expression analysis}

Tissue-specific RNAseq data were retrieved from the tomato functional genomics database (http://ted.bti.cornell.edu) for the tomato cultivar Heinz, and from the GEO database (https://www.ncbi.nlm.nih.gov.geo.query/acc.cgi?acc=GSE33507) for the tomato cultivar Moneymaker $(\mathrm{MM})^{45}$.

To confirm the expression of the tomato chitin receptor candidate genes, shoot tissue was collected from nine tomato $\mathrm{cv}$. Moneymaker plants infected with Verticillium dahliae strain JR2 at 4, 8 and 12 days post inoculation (dpi). Tissues from three plants were pooled for RNA isolation using the TRIZOL reagent (Invitrogen) according to manufacturer's instructions. Expression of SILYK4, SICEBiP, and tomato tubulin (SITUB) was analysed by real-time PCR as described previously ${ }^{36}$ using primer pairs shown in Supplementary Table 1. RNA was used as template to confirm the absence of contamination by genomic DNA. Expression levels in shoot tissues of Verticillium-infected plants were calculated relative to SITUB using the $\mathrm{E}^{-\Delta \mathrm{Ct}}$ method ${ }^{46}$ and normalized to mock-treated plants. 


\section{Virus-induced gene silencing of receptor candidates}

Selected sequences of SILYK4 and SICEBiP were amplified from tomato cv. Moneymaker CDNA and cloned into the pTRV2 vector ${ }^{47}$ using the Gateway technology (for primer sequences see Supplementary Table 1). Constructs were confirmed by sequencing (Supplementary Fig. 4) and transformed into Agrobacterium tumefaciens strain GV3101 by electroporation. Control TRV:GUS and TRV:PDS vectors had been previously generated and transformed into A. tumefaciens ${ }^{48}$. To silence SILYK4 and SICEBiP, cotyledons of 10 day-old tomato $\mathrm{cV}$. Moneymaker seedlings were infiltrated with 1:1 mixtures of pTRV1 and pTRV2 constructs $^{49}$. Photobleaching was observed 10-14 days after agroinfiltration of TRV:PDS. At $21 \mathrm{dpi}$ leaf tissue was harvested for physiological assays.

\section{Oxidative burst and chitin-responsive gene expression assays}

Oxidative burst measurements were performed on eight leaf discs from TRV-treated tomato plants as previously described ${ }^{50}$. Water was replaced with $100 \mu \mathrm{L}$ of a solution containing $100 \mu \mathrm{M} \mathrm{L}-012$ substrate and $20 \mu \mathrm{g} / \mathrm{mL}$ horseradish peroxidase. Luminescence was measured following treatment with $10 \mu \mathrm{M}$ chitohexaose (GN6; Megazyme) or water over 30 min using a CLARIOstar ${ }^{\circledR}$ microplate reader (BMG LABTECH). Leaf discs were kept in elicitor solutions for additional $30 \mathrm{~min}$ and then harvested and snap-frozen in liquid $\mathrm{N}_{2}$ for RNA extraction as described above. Chitin-responsive gene expression was tested as described previously ${ }^{36}$.

\section{Acknowledgements}

Parts of this work were carried out within the framework of the COST action FA1208 Sustain STSM fellowship program. Work in the laboratory of B.P.H.J.T. is supported by the Research Council for Earth and Life sciences (ALW) of the Netherlands Organization for Scientific Research (NWO).

\section{Author contributions}

H.R. and B.P.H.J.T. conceived the project. H.R., E.K.P. and B.P.H.J.T. designed the experiments. H.R., G.C.M.B., M.E., and O.V. performed the experiments and analyzed the data. H.R. and B.H.P.J.T. wrote the manuscript. 


\section{References}

1 Cook, D. E., Mesarich, C. H. \& Thomma, B. P. H. J. Understanding plant immunity as a surveillance system to detect invasion. Annu. Rev. Phytopathol. 53, 541-563, doi:10.1146/annurev-phyto-080614-120114 (2015).

2 Zipfel, C. \& Oldroyd, G. E. Plant signalling in symbiosis and immunity. Nature 543, 328-336, doi:10.1038/ nature22009 (2017).

3 Boller, T. \& Felix, G. A renaissance of elicitors: perception of microbe-associated molecular patterns and danger signals by pattern-recognition receptors. Annu. Rev. Plant. Biol. 60, 379-406, doi:10.1146/ annurev.arplant.57.032905.105346 (2009).

4 Sánchez-Vallet, A., Mesters, J. R. \& Thomma, B. P. H. $J$. The battle for chitin recognition in plant-microbe interactions. FEMS Microbiol. Rev. 39, 171-183, doi:10.1093/femsre/fuu003 (2015).

5 Fesel, P. H. \& Zuccaro, A. B-glucan: Crucial component of the fungal cell wall and elusive MAMP in plants. Fungal Genet. Biol., doi:10.1016/j. fgb.2015.12.004 (2015).

6 Felix, G., Regenass, M. \& Boller, T. Specific perception of subnanomolar concentrations of chitin fragments by tomato cells - induction of extracellular alkalinization, changes in protein-phosphorylation, and establishment of a refractory state. Plant J. 4, 307-316, doi:DO 10.1046/j.1365-313X.1993.04020307.x (1993).

7 Ren, Y. Y. \& West, C. A. Elicitation of diterpene biosynthesis in rice (Oryza sativa L.) by chitin. Plant Physiol. 99, 1169-1178 (1992).

8 Oldroyd, G. E. Speak, friend, and enter: signalling systems that promote beneficial symbiotic associations in plants. Nat. Rev. Microbiol. 11, 252263, doi:10.1038/nrmicro2990 (2013).

9 Genre, A. et al. Short-chain chitin oligomers from arbuscular mycorrhizal fungi trigger nuclear $\mathrm{Ca}^{2+}$ spiking in Medicago truncatula roots and their production is enhanced by strigolactone. New Phytol. 198, 179-189, doi:10.1111/nph.12146 (2013).

10 Gust, A. A., Willmann, R., Desaki, Y., Grabherr, H. M. \& Nürnberger, T. Plant LysM proteins: modules mediating symbiosis and immunity. Trends Plant Sci. 17, 495-502, doi:10.1016/j.tplants.2012.04.003 (2012).
11 Arrighi, J. F. et al. The Medicago truncatula lysin motif-receptor-like kinase gene family includes NFP and new nodule-expressed genes. Plant Physiol. 142, 265-279, doi:10.1104/pp.106.084657 (2006).

12 Cao, Y. et al. The kinase LYK5 is a major chitin receptor in Arabidopsis and forms a chitininduced complex with related kinase CERK1. Elife, doi:10.7554/eLife.03766 (2014).

13 Wan, J. et al. LYK4, a lysin motif receptor-like kinase, is important for chitin signaling and plant innate immunity in Arabidopsis. Plant Physiol. 160, 396-406, doi:10.1104/pp.112.201699 (2012).

14 Erwig, J., Ghareeb, H., Kopischke, M., Hacke, R., Matei, A., Petutschnig, E.K., Lipka, V. Chitininduced and CHITIN ELICITOR RECEPTOR KINASE1 (CERK1) phosphorylation-dependent endocytosis of Arabidopsis thaliana LYSIN MOTIF-CONTAINING RECEPTOR-LIKE KINASE5 (LYK5). New Phytol. 215, 382-396, doi:10.1111/nph.14592 (2017).

15 Shimizu, T. et al. Two LysM receptor molecules, CEBiP and OsCERK1, cooperatively regulate chitin elicitor signaling in rice. Plant J. 64, 204-214, doi:10.1111/j.1365-313X.2010.04324.x (2010).

16 Hayafune, M. et al. Chitin-induced activation of immune signaling by the rice receptor CEBiP relies on a unique sandwich-type dimerization. Proc. Natl. Acad. Sci. USA 111, E404-413, doi:10.1073/ pnas.1312099111 (2014).

17 Kaku, H. et al. Plant cells recognize chitin fragments for defense signaling through a plasma membrane receptor. Proc. Natl. Acad. Sci. USA 103, 11086-11091, doi:10.1073/pnas.0508882103 (2006).

18 Miyata, K. et al. The bifunctional plant receptor, OSCERK1, regulates both chitin-triggered immunity and arbuscular mycorrhizal symbiosis in rice. Plant Cell Physiol. 55, 1864-1872, doi:10.1093/ pcp/pcu129 (2014).

19 Faulkner, C. et al. LYM2-dependent chitin perception limits molecular flux via plasmodesmata. Proc. Natl. Acad. Sci. USA 110, 9166-9170, doi:10.1073/pnas.1203458110 (2013).

20 Narusaka, Y. et al. Presence of LYM2 dependent but CERK1 independent disease resistance in Arabidopsis. Plant Signal. Behav. 8, doi:10.4161/ psb.25345 (2013). 
21 Liu, B. et al. Lysin motif-containing proteins LYP4 and LYP6 play dual roles in peptidoglycan and chitin perception in rice innate immunity. Plant Cell 24, 3406-3419, doi:10.1105/tpc.112.102475 (2012).

22 Willmann, R. et al. Arabidopsis lysin-motif proteins LYM1 LYM3 CERK1 mediate bacterial peptidoglycan sensing and immunity to bacterial infection. Proc. Natl. Acad. Sci. USA 108, 1982419829, doi:10.1073/pnas.1112862108 (2011).

23 Ao, Y. et al. OsCERK1 and OsRLCK176 play important roles in peptidoglycan and chitin signaling in rice innate immunity. Plant J. 80, 1072-1084, doi:10.1111/tpj.12710 (2014).

24 Gust, A. A. Peptidoglycan perception in plants. PLoS Pathog. 11, e1005275, doi:10.1371/journal. ppat.1005275 (2015).

25 Zhang, X.W.etal. The receptor kinase CERK1 has dual functions in symbiosis and immunity signalling. Plant J. 81, 258-267, doi:10.1111/tpj.12723 (2015).

26 Limpens, E. \& Bisseling, T. Signaling in symbiosis. Curr. Opin. Plant Biol. 6, 343-350 (2003).

27 Limpens, E., van Zeijl, A. \& Geurts, R. Lipochitooligosaccharides modulate plant host immunity to enable endosymbioses. Annu. Rev. Phytopathol. 53, 311-334, doi:10.1146/annurevphyto-080614-120149 (2015).

28 Radutoiu, S. et al. Plant recognition of symbiotic bacteria requires two LysM receptor-like kinases. Nature 425, 585-592, doi:10.1038/nature02039 (2003).

29 op den Camp, R. et al. LysM-type mycorrhizal receptor recruited for rhizobium symbiosis in nonlegume Parasponia. Science 331, 909-912, doi:10.1126/science.1198181 (2011).

30 Buendia, L., Wang, T., Girardin, A. \& Lefebvre, B. The LysM receptor-like kinase SILYK10 regulates the arbuscular mycorrhizal symbiosis in tomato. New Phytol., doi:10.1111/nph.13753 (2015).

31 Zeng, L., Velasquez, A. C., Munkvold, K. R., Zhang, J. \& Martin, G. B. A tomato LysM receptor-like kinase promotes immunity and its kinase activity is inhibited by AvrPtoB. Plant J. 69, 92-103, doi:10.1111/j.1365-313X.2011.04773.x (2012).

32 Petutschnig, E. K., Jones, A. M., Serazetdinova, L., Lipka, U. \& Lipka, V. The lysin motif receptor-like kinase (LysM-RLK) CERK1 is a major chitin-binding protein in Arabidopsis thaliana and subject to chitininduced phosphorylation. J. Biol. Chem. 285, 2890228911, doi:10.1074/jbc.M110.116657 (2010).
33 Fliegmann, J. et al. Biochemical and phylogenetic analysis of CEBiP-like LysM domain-containing extracellular proteins in higher plants. Plant Physiol. Biochem. 49, 709-720, doi:10.1016/j. plaphy.2011.04.004 (2011).

34 Liu, S. et al. Molecular mechanism for fungal cell wall recognition by rice chitin receptor OsCEBiP. Structure 24, 1192-1200, doi:10.1016/j. str.2016.04.014 (2016).

35 Shinya, T., Nakagawa, T., Kaku, H. \& Shibuya, N. Chitin-mediated plant-fungal interactions: catching, hiding and handshaking. Curr. Opin. Plant Biol. 26, 64-71, doi:10.1016/j.pbi.2015.05.032 (2015).

36 de Jonge, R. et al. Conserved fungal LysM effector Ecp6 prevents chitin-triggered immunity in plants. Science 329, 953-955, doi:10.1126/ science.1190859 (2010).

37 Wan, J. R. et al. A LysM receptor-like kinase plays a critical role in chitin signaling and fungal resistance in Arabidopsis. Plant Cell 20, 471-481, doi:10.1105/tpc.107.056754 (2008).

38 Antolín-Llovera, M. et al. Knowing your friends and foes - plant receptor-like kinases as initiators of symbiosis or defence. New Phytol. 204, 791-802, doi:10.1111/nph.13117 (2014).

39 Baureithel, K., Felix, G. \& Boller, T. Specific, highaffinity binding of chitin fragments to tomato cells and membranes J. Biol. Chem. 269, 1793117938 (1994).

40 Rabea, E. I., Badawy, M. E., Stevens, C. V., Smagghe, G. \& Steurbaut, W. Chitosan as antimicrobial agent: applications and mode of action. Biomacromolecules 4, 1457-1465, doi:10.1021/ bm034130m (2003).

41 Iriti, M. \& Faoro, F. Chitosan as a MAMP, searching for a PRR. Plant Signal. Behav. 4, 66-68 (2009).

42 Shinya, T. et al. Functional characterization of CEBiP and CERK1 homologs in Arabidopsis and rice reveals the presence of different chitin receptor systems in plants. Plant Cell Physiology 53, 1696-1706, doi:10.1093/pcp/pcs113 (2012).

43 Kouzai, Y. et al. CEBiP is the major chitin oligomerbinding protein in rice and plays a main role in the perception of chitin oligomers. Plant Mol. Biol. 84, 519-528, doi:10.1007/s11103-013-0149-6 (2014).

44 Dereeper, A. et al. Phylogeny.fr: robust phylogenetic analysis for the non-specialist. Nucleic Acids Res. 36, W465-469, doi:10.1093/nar/ gkn180 (2008). 
45 Tomato Genome, C. The tomato genome sequence provides insights into fleshy fruit evolution. Nature 485, 635-641, doi:10.1038/ nature11119 (2012).

46 Livak, K. J. \& Schmittgen, T. D. Analysis of relative gene expression data using real-time quantitative PCR and the $2^{-\Delta \Delta C T}$ method. Methods 25, 402-408, doi:10.1006/meth.2001.1262 (2001).

47 Liu, Y., Schiff, M. \& Dinesh-Kumar, S. P. Virusinduced gene silencing in tomato. Plant J. 31, 777786 (2002).

48 Liebrand, T. W. et al. Endoplasmic reticulumquality control chaperones facilitate the biogenesis of $\mathrm{Cf}$ receptor-like proteins involved in pathogen resistance of tomato. Plant Physiol. 159, 1819-1833, doi:10.1104/pp.112.196741 (2012).
49 van der Hoorn, R. A. L., Laurent, F., Roth, R. \& de Wit, P. J. G. M. Agroinfiltration is a versatile tool that facilitates comparative analyses of Avr9/ Cf-9-induced and Avr4/Cf-4-induced necrosis. Mol. Plant-Microbe Interact. 13, 439-446, doi:Doi 10.1094/Mpmi.2000.13.4.439 (2000).

50 Gimenez-lbanez, S. et al. AvrPtoB targets the LysM receptor kinase CERK1 to promote bacterial virulence on plants. Curr. Biol. 19, 423-429, doi:10.1016/j.cub.2009.01.054 (2009). 


\section{Supplementary data}

$>$ SOI yc02g089900.1.1 | mature SILYK4

QQPYFGT GT NDCSSQDTSTSAF GYLCNGVNRT CQSYLTFRS QPPFNTVSSI SSLLGANPSQL SQLNSV SQNATF NTNQMVL VPVTCSCSGQF YQSNASYVI RRDDSFLNI AMNTLQGLSTCQAI NAENSEQANNLV VGSRI NVPLRCACPTQNQTNNGTNYLLTYLI ASGEFVSFI SDKFGVDFRATLAANSI PEDAPTVFPNT TLLVPLSTPPLSSQVAGPSPPPPPATTPTPPAVPVSESSSNKT WI YVVAGVVGGLVALCI LGVVVFFL FFRKKEKKADPQFVSESFEAVEKPSNKKVEEESEEFLESLSSI ĀQSVKVYKFEEVKAATENFSPTCLI KGSVYRGTI NGDFAAI KKMSGDVSKEI NLLSKI NHF NLI SLSGI CFHDGHWYLVYEYAANGPLSDWI C HHNGEQKSLSWAQRVQI SF DVATGLNYLHSYTSPPHVHKDL NGDNI LLDGDLRAKI ANF GLARSADGQ EGEFALTRHI VGT QGYMAPEYLENGLVSPKLDVYALGVLLLEI LTGKEVSALYEGSNTNLAELLI PVL NDDNAKESLSNF VDPSLQGKYPVELAFAMVRLI DNCLMKDPSHRPNT DEI VQSVSRI MTATHS WETSF STSVSPHRLP

>SOIyC01g112080.2.1 I mature SICEBiP

SFSCTSPGTCDAI I DYTLPNATTFNAVKKLFNVKNLRSLLGVNNLPVNTPADEKLPANQTI KI PFPCL CRNGTGI ANKRPI YTVVAGDFLSHI VTDI FAGLFTVEELQRVNNI SNPNLI QPGDKLWI PLPCSCDDV DGEKVVHYGRLVSSGNSI EAI AQQYNVSQETLLRLNGLASPRELLAGAVLDVPLKACQSRVSNASLDY PLLVPNDTYI FTAANCVTCKCDAASNWT LQCQPSQI KSSL WKTCPSMQCQGLDNLYI GNVTDCNSTSC AYAGYSNQTI FTTNTQLTCPASDNSAF GMRPGTWI WNVI L VAVSSMVI VF

SUPPLEMENTARY FIGURE 1 | Coverage map of SILYK4 and SICEBiP peptides identified by mass spectrometry. Peptides identified by LC-MS and mapping to SILYK4 or SICEBiP protein sequences are highlighted in grey. The first amino acid residue of the transmembrane domain of SILYK4 is underlined (W248). 


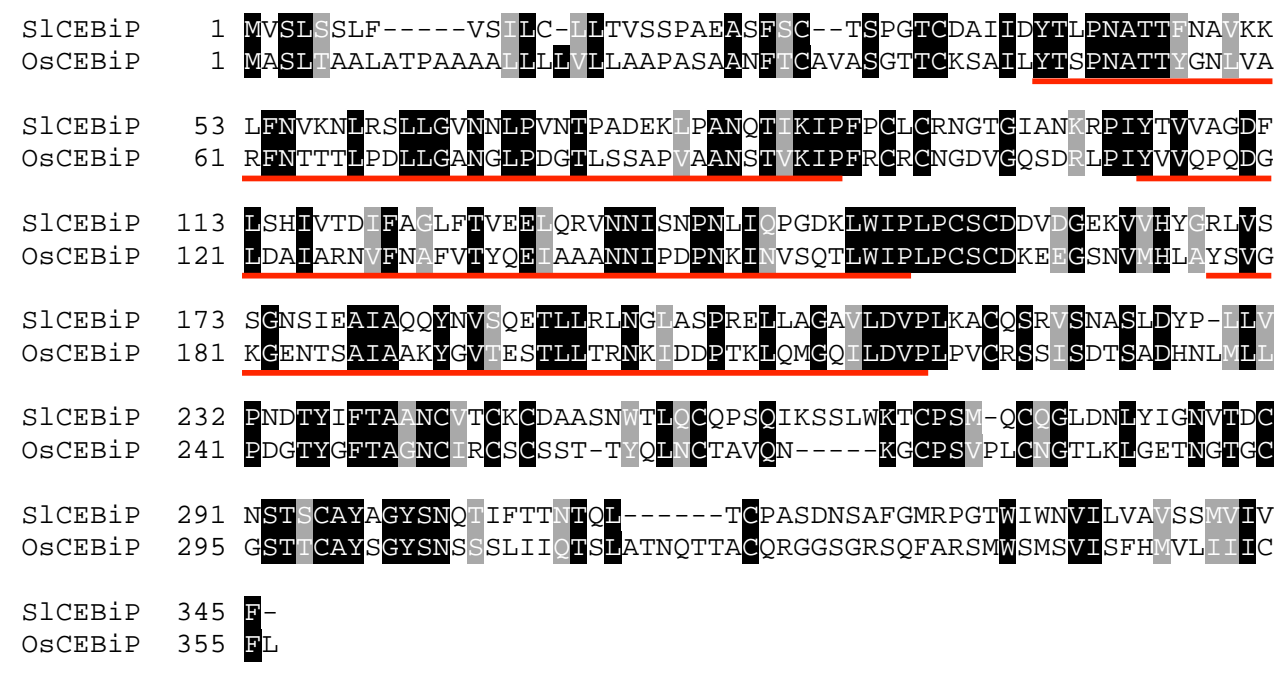

SUPPLEMENTARY FIGURE 2 | Pairwise alignment of rice and tomato CEBiP protein sequences. Full-length protein sequences of SICEBiP and OsCEBiP were aligned with Clustal Omega (http://www.ebi.ac.uk/Tools/msa/ clustalo/). LysM domains as annotated for OsCEBiP16,33 are underlined in red. Sequence conservation between the two receptors suggests that SICEBiP contains three LysM domains. 
a

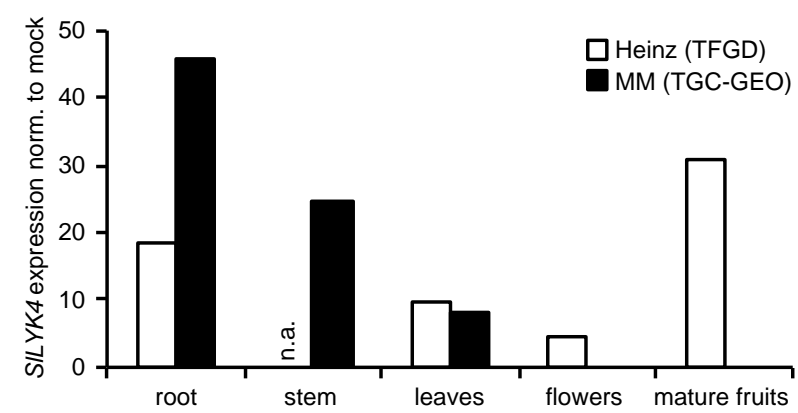

b

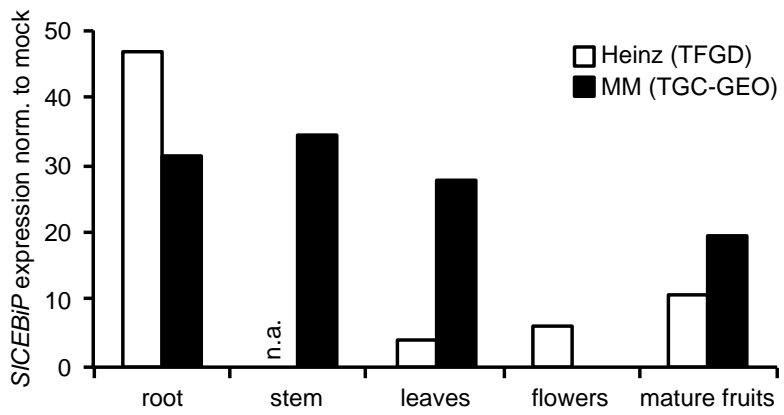

SUPPLEMENTARY FIGURE 3 | Expression of SILYK4 and SICEBiP in different tomato tissues. Tissue-specific expression patterns of SILYK4 (a) and SICEBiP (b) determined by RNAseq in the tomato cultivars Heinz 1706 and Moneymaker (MM) extracted from the tomato functional genomics database (TFGD) and the tomato genome consortium GEO (TGC-GEO) databases, respectively. 
a

SI CEBIP VIGS

SI CEBi P_t emp

SI CEBI P_VIGS

SI CEBi P_t emp

SI CEBIP_VIGS

SI CEBi P_t emp

SICEBIP_VIGS 130

SI CEBi P_ t emp

SI CEBI P VIGS

SI CEBi P_t emp

SICEBIP_VIGS 250

SI CEBi P_t t emp

SICEBIP_VIGS 310

SI CEBi P_ t emp

1

10
481 TCCCCTTTCCTTGTCTCTGTAGAAACGgTACCGGAATAGCCAACAAACGCCCCATTTACA

CCGTCGTCGCCGGCGACT TCCTATCGCACAT AGT TACCGACATCT TCGCCGGT TT TT TCA

CCGTCGTCGCCGGCGACTTCCTATCGCACATAGT TACCGACATCT TCGCCGGTTT GTTCA

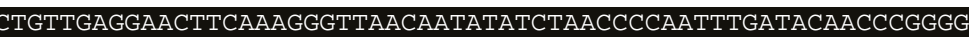

CT GT T GAGGAACT TCAAAGGGT TAACAATATATCTAACCCCAAT T T GATACAACCCGGGG

ATAAATT GT GGATCCCACT TCCT T GCAGCT GCGACGACGT T GACGGT GAAAAAGT TGTTC

ATAAAT T GT GGAT CCCACT T CCT T GCAGCT GCGACGACGT T GACGGT GAAAAAGT T GT TC

190 ATTATGGTCGATTGGTGAGCAGTGGCAACAGTATTGAGGCTATTGCTCAGCAGTACAATG

721 ATTATGGTCGATTGGTGAGCAGTGGCAACAGTATTGAGGCTATTGCTCAGCAGTACAATG

50 TTTCCCAGGAAACCCTCTTGAGGT GAATGGTTTAGCAAGTCCCAGAGAACTTTTAGCTG

IT TCCCAGGAAACCCTCT TGAGGT T GAAT GGT T TAGCAAGTCCCAGAGAACTTTTAGCT G

GCGCAGTTCTTGACGTTC

GCGCAGTTCTTGACGTTCCCCTTAAAGCTTGCCAATCAAGGGTGAGCAATGCCTCGCTGG

b

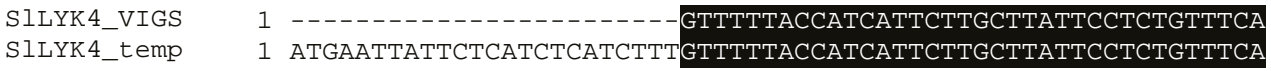

SILYK4 VIGS 37 ATTCTTGCACAACAGCCTTATTTTGGAACTGGAACAAATGACTGCAGCAGCCAAGATACC

SI LYK4_temp 61 ATTCTTGCACAACAGCCTTATTTTGGAACTGGAACAAATGACTGCAGCAGCCAAGATACC

SILYK4_VIGS 97 ICCACTTCTGCTTTTGGGTATTTATGCAATGGCGTTAACCGTACTTGCCAATCTTATTTG

SILYK4_te mp 121 TCCACTTCTGCTTTTGGGTATTATGCAATGGCGTTAACCGTACTTGCCAATCTTATTTG

SILYK4_VIGS 157 ACCTTCAGATCTCAACCCCCTTTCAATACTGTGTCCTCAATCTCTTCTTTACTCGGTGCT

SI LYK4_t e mp 181 ACCTTCAGATCTCAACCCCCTTTCAATACT GTGTCCTCAATCTCT TCT TTACTCGGTGCT

SILYK4 VIGS 217 AATCCTTCACAGCTCTCTCAGCTCAATTCTGTTTCTCAAAATGCTACCTTTAACACCAAT

SILYK4_te mp 241 AATCCTTCACAGCTCTCTCAGCTCAATTCTGTTTCTCAAAATGCTACCTTTAACACCAAT

SUPPLEMENTARY FIGURE 4 | Alignment of target sequences selected for virus-induced gene silencing with gene templates. Prior to transformation of $A$. tumefaciens pTRV2 vectors carrying gene fragments of SICEBiP (a) and SILYK4 (b) were verified by sequencing. 


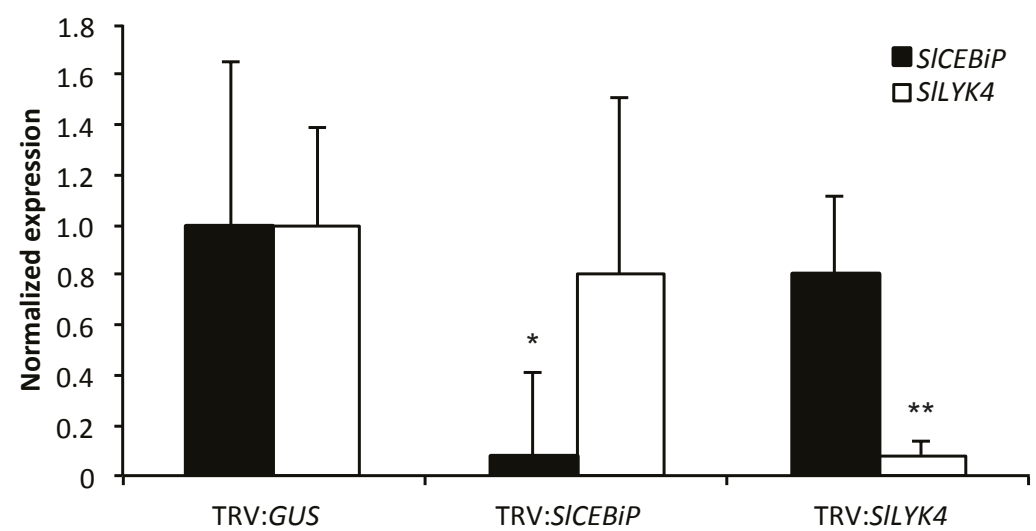

SUPPLEMENTARY FIGURE 5 | Efficiency and specificity of SILYK4 and SICEBiP silencing in tomato. Silencing efficiency of SICEBiP and SILYK4 was determined by qRT-PCR in tomato leaves three weeks following tobacco rattle virus (TRV) treatment using gene-specific primers. Primers targeting the tomato tubulin gene were used for sample calibration. Relative transcript levels were set to 1 for TRV:GUS-treated samples for each gene. Bars represent expression levels in $n>3$ plants \pm S.D.. Statistically significant differences in expression compared to TRV:GUS plants were determined with a Student's $t$ test $\left({ }^{*} p<0.5 ;{ }^{* *} p<0.01\right)$.

SUPPLEMENTARY TABLE 1 | Primers used in this study.

\begin{tabular}{|lll|}
\hline Name & Sequence $\left(\mathbf{5}^{\prime} \rightarrow \mathbf{3}^{\prime}\right)$ & Application \\
\hline SILYK4_qPCR_F & TCAACGCGGAGAACAGTGAA & qRT-PCR \\
SILYK4_qPCR_R & GCCCTAAAATCCACCCCAAA & qRT-PCR \\
SICEBiP_qPCR_F & CTTGCCAATCAAGGGTGAGC & qRT-PCR \\
SICEBiP_qPCR_R & ATCTGGGATGGTTGGCATTG & qRT-PCR \\
SITUB_F & AACCTCCATTCAGGAGATGTTT & qRT-PCR \\
SITUB_R & TCTGCTGTAGCATCCTGGTATT & qRT-PCR \\
SILYK4_VIGS_F & GGGGACAAGTTTGTACAAAAAAGCAGGCTTCATGAATTATTCTCATCTC & VIGS \\
SILYK4_VIGS_R & GGGGACCACTTTGTACAAGAAAGCTGGGTTTCATTATTAATTGGTGTTAAAGGTAGC & VIGS \\
SICEBiP_VIGS_F & GGGGACAAGTTTGTACAAAAAAGCAGGCTCCATTTACACCGTCGTCGC & VIGS \\
SICEBiP_VIGS_R & GGGGACCACTTTGTACAAGAAAGCTGGGTGAACGTCAAGAACTGCGCC & VIGS \\
\hline
\end{tabular}




\title{
Chapter 5
}

\section{Neofunctionalisation after horizontal gene transfer: birth of a fungal effector that evolved towards immune suppression}

\author{
Hanna Rovenich', Nick C. Snelders ${ }^{1 \#}$, Jordi C. Boshoven "\#, \\ Carolina Grandellis ${ }^{2, \$}$, Michael F. Seidl', Sjef Boeren ${ }^{3}$, Makoto Ogata $^{4}$, \\ Jorgelina Ottado ${ }^{2}$, Jeroen R. Mesters ${ }^{5}$, Bart P. H. J. Thomma ${ }^{1}$ \\ 'Laboratory of Phytopathology, Wageningen University, Droevendaalsesteeg 1, 6708 PB Wageningen, \\ The Netherlands; ${ }^{2}$ IBR-CONICET, CCT-Rosario, Ocampo y Esmeralda, 2000 Rosario, Argentina; \\ ${ }^{3}$ Laboratory of Biochemistry, Wageningen University, Stippeneng 4, 6708 WE Wageningen, The Netherlands; \\ ${ }^{4}$ Department of Chemistry and Biochemistry, National Institute of Technology, Fukushima College, \\ Fukushima 970-8034, Japan; ${ }^{5}$ Institute of Biochemistry, University of Lübeck, Center for Structural \\ and Cell Biology in Medicine, Ratzeburger Allee 160, 23538 Lübeck, Germany \\ \$Current address: The Sainsbury Laboratory, Norwich Research Park, Norwich NR4 7UH, United Kingdom
}




\begin{abstract}
Microbes utilize secreted molecules to facilitate niche establishment, including microbial pathogens that colonize plant hosts ${ }^{1}$. We previously identified the secreted effector protein Ave1 of the vascular wilt fungus Verticillium dahliae that acts as a virulence factor during plant colonization ${ }^{2}$. Ave1 homologs are ubiquitous in plants and occur only in few plant pathogens that acquired them via horizontal gene transfer (HGT)2. Intriguingly, all homologs carry a conserved plant natriuretic peptide (PNP) sequence. Here we show that, as previously demonstrated for some of its homologs, also V. dahliae Ave1 displays PNP activity. However, the contribution of Ave1 to virulence is not dependent on its PNP activity. Further analysis revealed that Ave1 interacts with plant endochitinases, which are important executors of plant defense ${ }^{3}$. Intriguingly, in contrast to its homologs, Ave1 inhibits endochitinases to interfere with host immunity. Thus, Ave1 functionally diverged from its microbial and plant homologs upon HGT from plants to evolve a novel function in immune suppression.
\end{abstract}




\section{Introduction}

Verticillium dahliae is a soil-inhabiting fungal pathogen that causes vascular wilt in over 200 dicotyledonous plants species, including many crops ${ }^{4,5}$. Like other microbes, $V$. dahliae secretes effector molecules to facilitate niche establishment ${ }^{1,6}$. Using comparative population genomics, we identified the $V$. dahliae effector gene Ave 1 (hereafter referred to as VdAve1) that is crucial for fungal aggressiveness during infection (virulence) on tomato (Solanum lycopersicum), on Arabidopsis thaliana ${ }^{2}$, and on other host plants (B.P.H.J.Thomma, unpublished data). Similarity searches revealed that VdAve1 has numerous homologs in plants, but also in the phytopathogenic fungi Fusarium oxysporum, Colletotrichum higginsianum, and Cercospora beticola, in the saprotroph Verticillium nubilum as well as in the bacterial pathogen Xanthomonas citri subsp. citri ${ }^{2,7}$. Since the distribution of VdAve1 homologs does not follow the phylogeny of the species in which they occur, it has been proposed that $V$. dahliae acquired VdAve1 from plants by horizontal gene transfer (HGT)2. This hypothesis is further supported by its localization in a highly dynamic lineage-specific region of the $V$. dahliae genome $e^{8-10}$.

\section{Results and discussion}

Most plant homologs of VdAve1 are annotated as plant natriuretic peptides (PNPs) ${ }^{11,12}$; functional analogues of vertebrate atrial natriuretic peptides (ANPs) that contribute to maintenance of osmotic and cardiovascular homeostasis ${ }^{13}$. Similarly, PNPs are systemically mobile molecules that are released under biotic and abiotic stress conditions and have been implicated in ion and water homeostasis ${ }^{12,14}$. Interestingly, the VdAve1 homolog XacPNP that is produced by the plant pathogenic bacterium $X$. citri subsp. citri is a horizontally acquired virulence factor that promotes bacterial proliferation through its PNP activity ${ }^{15,16}$. An alignment of VdAve1 with homologs of $V$. nubilum (VnAve1), C. beticola (CbAve1), F. oxysporum (FoAve1), C. higginsianum (ChAve1), XacPNP, tomato (SIAve1), and A. thaliana (AtPNP-A) shows that the smallest peptide with PNP function lies within the most conserved region (grey box, Fig $1 \mathrm{a}^{, 15}$ ). To test whether VdAve1 has PNP activity, its ability to promote stomatal opening was assayed in tomato (Fig. 1b). Treatment of leaf epidermis with VdAve1 resulted in significantly enhanced stomatal opening as similarly observed upon treatment with XacPNP and the synthetic auxin analogue naphthalene acetic acid (NAA) (Fig 1b). Consistent with previous reports demonstrating that PNP-induced responses are dependent on cyclic guanosine monophosphate (cGMP) signalling ${ }^{17}$, aperture changes caused by VdAve1 were partially repressed by the guanylate cyclase inhibitor methylene blue. Collectively, these findings demonstrate that VdAve1 displays PNP activity. 
To test whether the PNP activity accounts for the virulence activity of VdAve1, we complemented a VdAve1 deletion mutant $(\triangle V d A v e 1)^{2}$ with VnAve1, ChAve1, FoAve1, CbAve1, XacPNP, or SIAve1 under control of the VdAve1 promoter (Supplementary Fig. 1a), and confirmed transgene expression by qRT-PCR (Supplementary Fig. 1b). For each construct, we selected two transformants of which at least one reached an expression value similar to wild type VdAve 1 for inoculation assays on tomato. Surprisingly, none of the VdAve1 homologs was able to restore the virulence of the VdAve1 deletion mutant to wild type, as determined by assessment of symptom display of inoculated plants (Fig. 2a) and fungal biomass by qRT-PCR (Fig. 2b). Since the PNP domain is highly conserved in all homologs tested here and is functional in VdAve1 as well as XacPNP, these results suggest that the PNP activity of VdAve1 is not responsible for its role in virulence, and that this effector has acquired an additional function to facilitate host colonization.

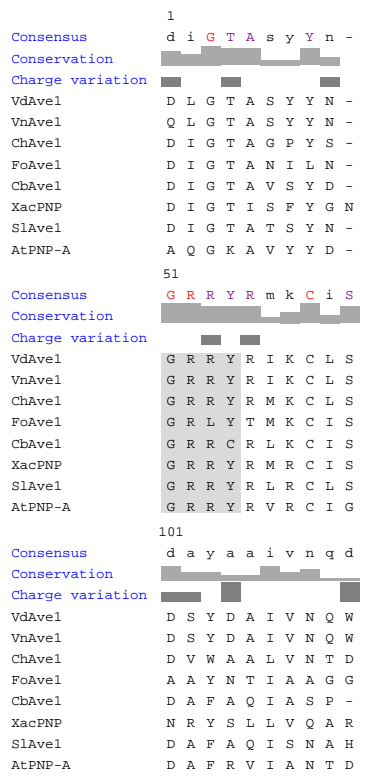

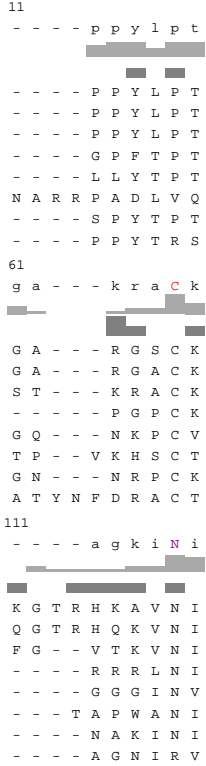

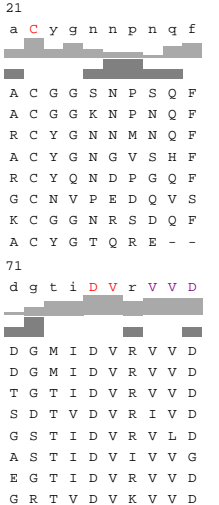

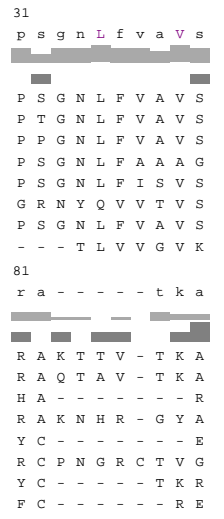
D $D G L W D N G A$ E G L W W D $D G G \quad L \quad W \quad D \quad N \quad G \quad A \quad A \quad C$ $G M W D N N G$ A A G L L W $D$ D N $N$ G A A A G L W D N G A S C E G L W D N N G A A A N N L W Q N G R A

t m i l s

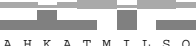
A H K A T M I L S A H K A T M I L 5 $\begin{array}{llllllllll}K & P & R & N & T & I & D & F & P & Q\end{array}$ - E F F L L H $M K L 5$

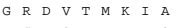
$\begin{array}{llllllllllll} & N & G & D & L & N & L & S\end{array}$

e y v a FIGURE 1 | The conserved PNP domain is active in VdAve1. a, Multiple sequence alignment of mature VdAve1 with homologs from Verticillium nubilum (Vn), Colletotrichum higginsianum (Ch), Fusarium oxysporum (Fo), Cercospora beticola $(\mathrm{Cb})$, Xanthomonas citri (Xac), tomato (SI) and Arabidopsis thaliana (At) shows highly conserved PNP domain (grey box). b. Stomatal opening was determined in tomato epidermis following treatment with 5 $\mu \mathrm{M}$ VdAve 1 or XacPNP in absence of presence of methylene blue (MB). Naphthalene acetic acid (NAA; $1 \mu \mathrm{M}$ ) and $50 \mu \mathrm{M}$ abscisic acid (ABA) were used as positive and negative controls, respectively. Data are from one representative experiment. Experiments were performed twice. Letters represent statistically significant differences in stomatal opening according to one-way ANOVA $(F(5,824)=124.8, \mathrm{p}<0.001)$ and Tukey's post-hoc test. Error bars represent the mean $\pm S D(n>70)$. 


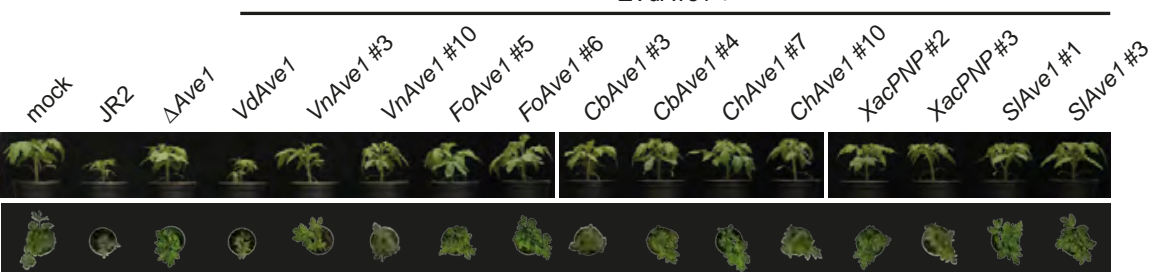

b
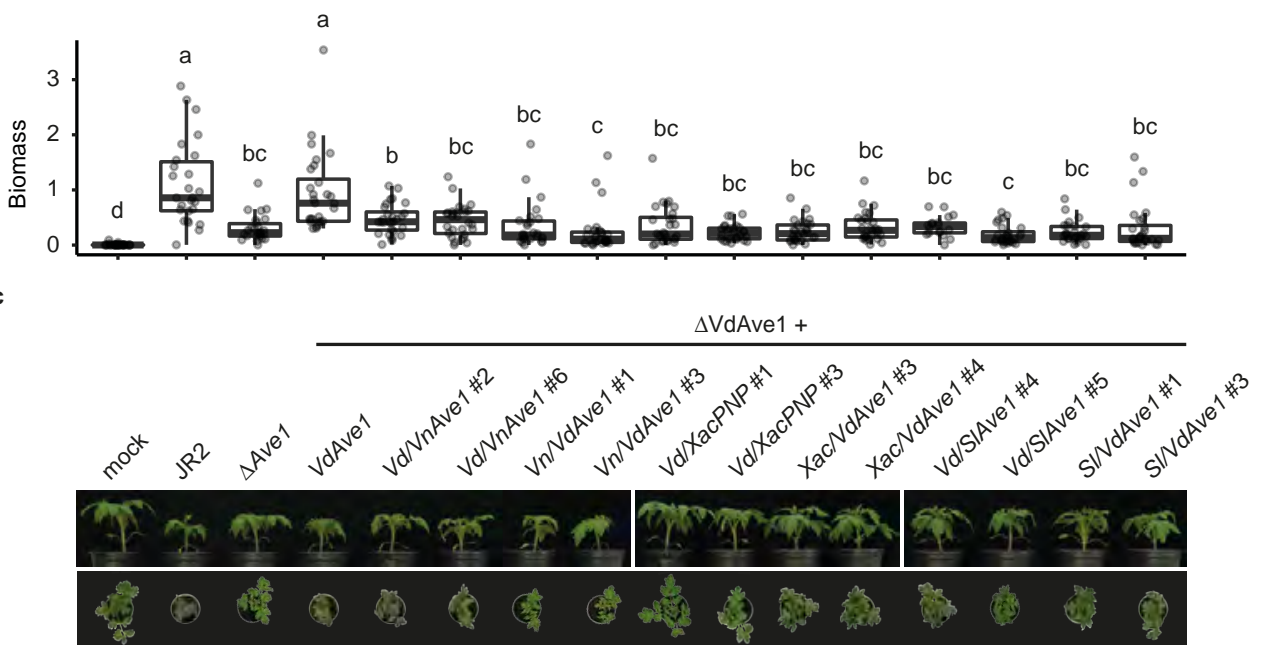

d

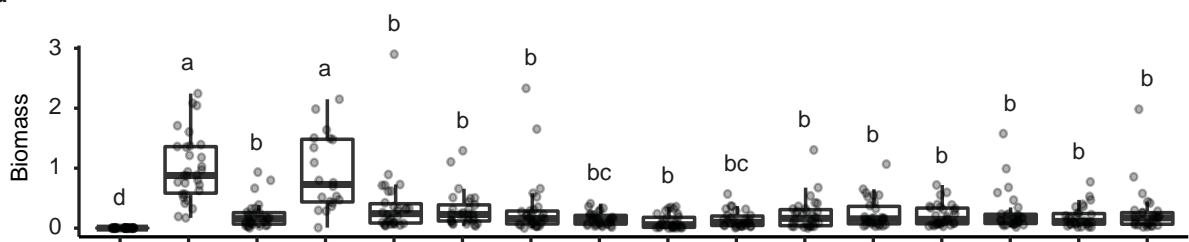

FIGURE 2 | VdAve1 has functionally diverged from plant and microbial homologs. Complementation assays in the V. dahliae Ave 1 deletion mutant with microbial and plant homologs (a,b; Supplementary Fig. 1) and chimeric mutants (c,d; Supplementary Fig. 2) of Ave1 show that full-length VdAve1 is required for $V$. dahliae virulence during tomato colonization. a, Representative stunting symptoms of plants inoculated with wild type $V$. dahliae (JR2), $\triangle V d A v e 1, \triangle V d A v e 1$ complemented with VdAve1, or two independent $\triangle V d A v e 1$ strains expressing VdAve1 homologs from V. nubilum (Vn), C. higginsianum (Ch), C. beticola (Cb), F. oxysporum (Fo), X. citri (Xac), and tomato (SI). The XacPNP sequence encoding the mature protein was fused to the VdAve1 signal peptide to ensure secretion. Plants inoculated with strains expressing VdAve1 homologs display stunting symptoms similar to plants inoculated with the VdAve1 deletion strain, suggesting that the homologs fail to reinstall virulence. b, Fungal biomass was quantified in stem tissue of all mock-treated and $V$. dahliae-inoculated plants by quantitative RT-PCR on genomic DNA targeting the $V$. dahliae ITS gene at 17-19 days after inoculation. Primers targeting the tomato rubisco gene were used for sample calibration. Data are from three independent experiments. Letters represent statistically significant differences in biomass according to one-way ANOVA $(F(15,399)=19.988, \mathrm{p}<0.001)$ and Tukey's post-hoc test. Error bars represent the mean $\pm S D(n>26)$. c, Similar to full-length homologs, complementation of the VdAve1 deletion mutant with constructs expressing chimeric Vn/VdAve1, Vd/VnAve1, Xac/VdAve1, Vd/XacPNP, SI/VdAve1 and Vd/SIAve1 did not result in the recovery of virulence. $\mathbf{d}$, Quantification of fungal biomass as in $\mathbf{b}$. Data are from three independent experiments. Letters represent statistically significant differences in biomass according to oneway ANOVA $(F(15,435)=67.084, p<0.001)$ and Tukey's post-hoc test. Error bars represent the mean \pm SD $(n>29)$. 
We then generated a range of constructs for the expression of domain swap mutants between VdAve1 and VnAve1, XacPNP or SIAve1 in the VdAve1 deletion mutant to investigate which portion of VdAve1 is responsible for its virulence function (Supplementary Fig. 2a). The swapping sites were chosen such that the most conserved sequence, including the active PNP domain, originated from the VdAve1 homologs. Again, transformants were selected based on transgene expression as described above (Supplementary Fig. 2b). Intriguingly, symptom assessment and quantification of fungal biomass in infected plants showed that, similar to the full-length homologs, none of the chimeric Ave1 proteins reinstalled virulence in $\Delta V d A v e 1$ during tomato colonization (Fig. 2c,d). This finding confirms that the PNP domain is not responsible for the virulence function of VdAve1. Moreover, we hypothesize that the domain swaps disrupted the domain required for the virulence activity of VdAve1. Based on the functional dissection of the VdAve1 epitope, we have recently proposed that the surface that is formed by the $\mathrm{N}$ - and C-termini of VdAve 1 is crucial for the recognition by the tomato immune receptor $\mathrm{Ve} 1^{7}$. Taken together, these findings suggest that a modification of either terminus of the protein destabilizes its overall structure, affecting both VdAve1 immune recognition and its virulence function.

Considering that the PNP activity is not responsible for the virulence contribution of VdAve1, and that we cannot attribute the virulence function to a specific subdomain of the effector protein, we pursued affinity purification and identification of interacting plant proteins by mass spectrometry to reveal how VdAve1 contributes to virulence. To this end, we transiently expressed VdAve1 fused to green fluorescent protein (GFP), which was previously shown to be recognized by Ve1, in tobacco (Nicotiana tabacum) leaves ${ }^{7}$. The transiently expressed proteins were immunopurified with aGFP agarose beads and peptides generated by tryptic on-bead digestion were analyzed with mass spectrometry. While twelve proteins specifically co-purified with VdAve1, only two tobacco endochitinases ( $\mathrm{CHI} 1$ and $\mathrm{CHI} 2$ ), with an average of 4 unique peptides in four biological replicates, were considered as bona fide interactors, since the remaining proteins are predicted to be localized intracellularly while VdAve1 is thought to act extracellularly (Fig. 3a; Supplementary Fig. 3; Supplementary Table 2). Endochitinases are important pathogenesis-related proteins and play a key role in plant defense against fungal microbes ${ }^{3,18}$. Consequently, several mechanisms evolved in fungi that enable them to evade or suppress host chitinase activity, such as proteolytic cleavage of chitinases and competition for the binding of chitinase substrates ${ }^{19-21}$. 


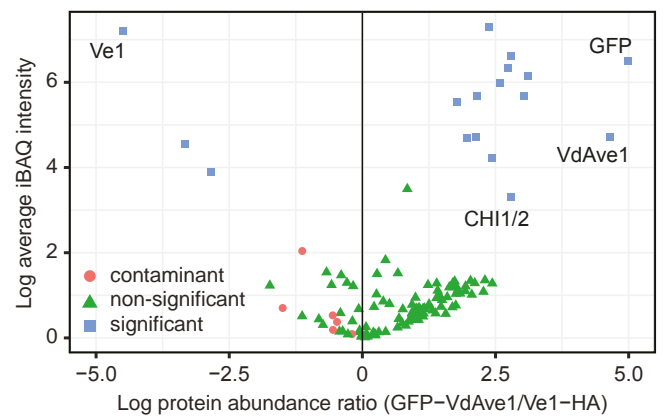

b

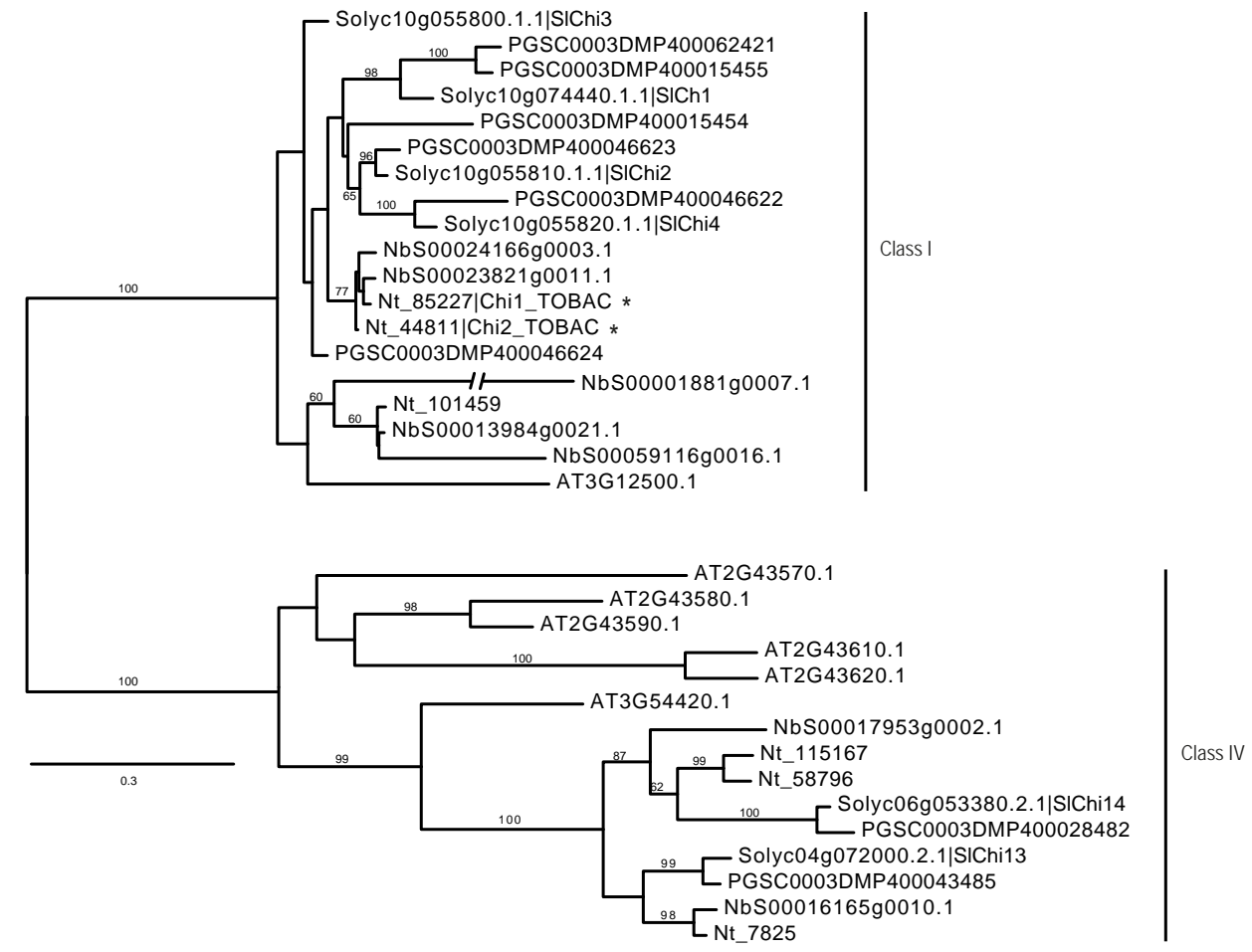

FIGURE 3 | ChtBD1 domain-carrying tomato endochitinases are putative targets of VdAve1. a, Putative targets of VdAve1 were identified by mass spectrometry following transient expression of GFP-tagged VdAve1 in tobacco leaves. Leaves of four plants were independently infiltrated with A. tumefaciens carrying GFP-Ave1 or Ve1-HA constructs and treated as biological replicates in subsequent analyses. Immunopurification of tagged proteins was carried out on total leaf extracts 2 days after infiltration. The secreted tobacco endochitinases $\mathrm{CHI} 1$ and CHI2 specifically co-purified with GFP-Ave1. b, Phylogeny of putative secreted endochitinases of tobacco (Nicotiana tabacum), tomato (Solanum lycopersicum), Nicotiana benthamiana, potato (Solanum tuberosum), and Arabidopsis thaliana carrying both a chitin-binding (ChtBD1; PF00187) and a glycosyl hydrolase family 19 (GH19; PF00182) domain. Clustering shows that endochitinases group into two clades corresponding to class I and class IV chitinases. The two tobacco chitinases $\mathrm{CHI} 1$ and $\mathrm{CHI} 2\left(^{*}\right)$ identified by mass spectrometry belong to class I, which contains four homologs from tomato. The clade of class IV chitinases only contains two putative tomato endochitinases. 
The tomato genome comprises six genes encoding endochitinases (SIChi1, S/Chi2, SIChi3, SIChi4, SIChi13, and SIChi14) that are closely related to tobacco CHI1 and CHI2. These are the only secreted tomato chitinases that contain both a chitin-binding type-1 (ChtBD1; PF00187) and a chitinolytic glycosyl hydrolase 19 (GH19; PF00182) domain ${ }^{21}$. The ability to bind chitin via their ChtBD1 domain has previously been described to enhance enzymatic efficiency of GH19 chitinases, and removal of this domain compromises their enzymatic and antifungal activity ${ }^{21-23}$. Phylogenetic analysis of the tobacco and tomato chitinases and predicted secreted chitin-binding chitinases from Nicotiana benthamiana, Solanum tuberosum (potato), and Arabidopsis thaliana demonstrated that they group into two clades corresponding to class I and class IV chitinases (Fig. 3b) ${ }^{24}$. Quantitative RT-PCR analysis showed that all six endochitinase genes are expressed in tomato stem tissue during V. dahliae infection (Supplementary Fig. 4). Similarly, expression of S/Chi2, SIChi3, SIChi4, and SIChi13 was induced during tomato infection by F. oxysporum that, like $V$. dahliae, colonizes the plant vascular system ${ }^{21,25}$. Additionally, SIChi2, SIChi3, and SIChi4 expression was significantly upregulated in tomato leaf tissue upon inoculation with the extracellular, foliar pathogen Cladosporium fulvum ${ }^{21,26,27}$. These results indicate that the six chitin-binding tomato chitinases play an important role in defense against various fungal pathogens.

The finding that VdAve1 interacts with chitinases may suggest that $V$. dahliae utilizes this effector to inhibit host chitinases in order to protect itself from their enzymatic activity. To determine the potential inhibitory activity of VdAve1, we purified the tomato class I chitinases SIChi2 and SIChi4 upon heterologous expression from Pichia pastoris, and SIChi13 as representative of class IV chitinases. We first assessed the hydrolytic activity of SIChi2, SIChi4, and SIChi13 alone using a colorimetric assay. Of the three enzymes, only SIChi4 displayed endochitinolytic activity (Supplementary Fig. 5a) while its ability to hydrolyze the exochitinase-specific substrate was negligible, confirming its annotation as endochitinase. Neither SIChi2 nor SIChi13 showed measurable enzymatic activity, possibly indicating differences in substrate specificity as both chitinases were previously shown to hydrolyze insoluble chitin azure ${ }^{21}$. Based on these findings, SIChi4 was used in subsequent experiments. Its activity was suppressed by the chitinase inhibitor tri-Nacetylchitotriosyl moranoline $\left(\mathrm{GN}_{3} \mathrm{M}\right)^{28}$ in a concentration-dependent manner, but not by monomeric $\mathrm{N}$-acetylchitotriosyl moranoline (GNM) ${ }^{28}$ (Supplementary Fig. 5b,c), which were used as positive and negative controls, respectively. Interestingly, incubation of SIChi4 with Escherichia coli-produced VdAve1 resulted in significantly decreased SIChi4 activity, demonstrating that the effector indeed acts as a chitinase inhibitor (Fig. 4). Notably, $0.5 \mu \mathrm{M}$ VdAve1 was sufficient to reduce SIChi4 activity by $~ 50 \%$, which is similar to the inhibition by $100 \mu \mathrm{M} \mathrm{GN}{ }_{3} \mathrm{M}$, demonstrating that VdAve1 is a considerably more potent chitinase inhibitor than $\mathrm{GN}_{3} \mathrm{M}$. 


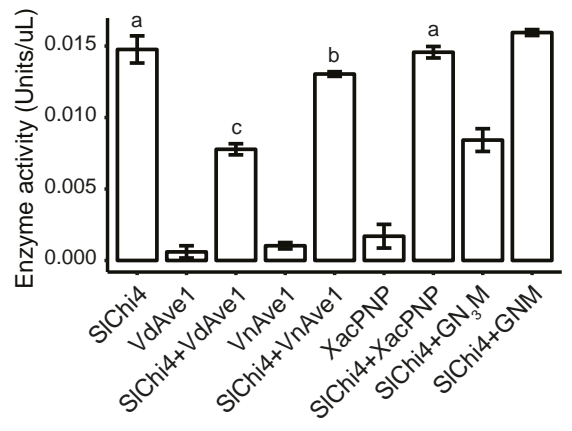

FIGURE 4 | VdAve1 inhibits tomato endochitinase activity. SIChi4 $(0.1 \mu \mathrm{M})$ activity was quantified based on the release of $p$-nitrophenol from the endochitinase substrate 4-Nitrophenyl $\beta-D-N, N^{\prime}, N^{\prime \prime}$-triacetylchitotriose following two hours of incubation in reaction buffer $\mathrm{pH}$ 5.5) at $37^{\circ} \mathrm{C}$. For inhibition, SIChi4 was pre-incubated for 15 min with $0.5 \mu \mathrm{M}$ effector proteins or $100 \mu \mathrm{M} \mathrm{GN} \mathrm{N}_{3} \mathrm{M}$ and GNM, respectively. Data are from three independent experiments with two technical replicates. Bars represent means $\pm \mathrm{SD}(n=3)$. Letters show statistically significant differences according to one-way ANOVA and Tukey's post-hoc test.

Since complementation of the VdAve1 deletion mutant of $V$. dahliae with homologs from microbes and plants did not result in the recovery of virulence, we speculated that only VdAve1 has the capacity to inhibit endochitinase activity. To validate this hypothesis, we assessed the inhibitory activity of selected Ave1 homologs towards SIChi4. To this end, we tested the closest VdAve1 homolog, VnAve1, as well as the well-characterized homolog XacPNP, which was shown to act as a microbial virulence factor. In support of our hypothesis, only addition of VdAve1, but not of VnAve1 or XacPNP to the reaction mixture resulted in significant inhibition of SIChi4 activity (Fig. 4). Collectively, these results demonstrate that only VdAve1, in contrast to its homologs, acquired an additional functionality to suppress the hydrolytic activity of plant endochitinases.

The hydrolytic cleavage of cell wall chitin by endochitinases during plant colonization has detrimental effects on invading fungal microbes. On the one hand, it releases chitin fragments that are recognized by plant cell surface receptors, resulting in the stimulation of further immune responses ${ }^{29}$. On the other hand, the hydrolysis of structural chitin macromolecules affects the integrity of the fungal cell wall, which may ultimately lead to cell collapse ${ }^{18}$. Thus, by secreting VdAve1 during host colonization, V. dahliae may interfere with chitin-triggered immunity in two, not mutually exclusive, ways: (1) by inhibiting the hydrolysis of fungal hyphae, and (2) by preventing the release of immunogenic chitin oligomers. This finding explains the earlier observation that the core chitin-binding lysin motif (LysM)-containing effector proteins produced by $V$. dahliae do not interfere with chitin-triggered immunity ${ }^{30}$. This was surprising since all fungal plant pathogens need to address the threat posed by chitin-triggered host immunity, a role that has been attributed to the ubiquitous fungal LysM effectors in many species ${ }^{20,31,32}$. However, in $V$. dahliae, VdAve1 has evolved to fulfill this task.

The acquisition of effector genes by HGT from plants has been associated with emergence of pathogenicity and microbial host range expansion ${ }^{33}$. Moreover, phytopathogens utilize host-related molecules for immune evasion and host manipulation ${ }^{15,34}$. Here we show that VdAve1, in contrast to its homologs, inhibits tomato endochitinases during infection, representing a novel strategy for phytopathogens to deal with host-derived cell 
wall hydrolytic enzymes. The finding of yet another strategy to undermine chitin-triggered immunity underpins its importance in host defense against fungal pathogens. Thus, we reveal a case of neofunctionalisation of a horizontally acquired effector gene toward a function in host immune suppression.

\section{Methods}

\section{Sequence alignment of Ave1 proteins}

Protein alignment was performed using MAFFT (Version 7.271) ${ }^{35}$.

\section{Production and purification of recombinant effector proteins}

The sequences encoding mature VdAve1, VnAve1, and XacPNP were cloned into the pET$15 \mathrm{~b}$ expression vector for $\mathrm{N}$-terminal His tagging (Novagen, Madison, WI, USA) (for primer sequences see Supplementary Table 1). Heterologous proteins were produced and purified from inclusion bodies under denaturing conditions using His60 $\mathrm{Ni}^{2+}$ Superflow Resin (Clontech, Mountain View, CA, USA). Purified proteins were stored in $0.25 \mathrm{M}$ ammonium sulphate with $0.1 \mathrm{M}$ BisTris, $\mathrm{pH}$ 5.5. Final concentrations were determined using the BioRad Protein Assay (BioRad, Veenendaal, The Netherlands). For details see Supplementary Methods.

\section{Stomatal opening assay}

Stomatal aperture was tested as described previously ${ }^{15}$ using tomato leaf tissue. Significant differences between treatments were determined using one-way ANOVA followed by Tukey's post-hoc test in $\mathrm{R}$ (Version 3.3.2).

\section{Generation of $V$. dahliae complementation strains}

Genes encoding VdAve1 homologs and chimeric Ave1 proteins (Supplementary Fig. 2) were synthesized (Eurofins, Ebersberg, Germany) and cloned into the pFBT005 vector under the control of the VdAve 1 promoter (for details see Supplementary information). For expression of full-length XacPNP and XacNdAve1, the native signal peptide of XacPNP was replaced with that of VdAve1 (see Supplementary Fig. 1a, 2a). Transformation of V. dahliae $\triangle A v e 1^{2}$ with $A$. tumefaciens Agl 1 carrying the different constructs was performed as described previously ${ }^{7,36,37}$. Transgene expression was determined for individual colonies, grown in liquid $1 / 2$ Murashige \& Skoog medium supplemented with 3\% sucrose and $1 \mathrm{mM} \mathrm{MES,} \mathrm{pH} 5.7$ at $22^{\circ} \mathrm{C}$ and $120 \mathrm{rpm}$, by qRT-PCR using primers targeting specific transgenes and VdGAPDH for sample calibration (Supplementary Table 1). Quantitative RT-PCR conditions and analyses of results were described previously². 


\section{Disease assays}

Tomato inoculations were carried out as described previously ${ }^{36}$. Disease development was monitored up to 19 days after inoculation. $V$. dahliae biomass was quantified as described in the Supplementary Methods. Significant differences in biomass between tomato plants inoculated with $V$. dahliae wild type and mutant strains was determined by one-way ANOVA, followed by Tukey's post-hoc analysis in R (Version 3.3.2).

\section{VdAve1 target identification by immunopurification and mass spectrometry.}

Immunopurifications were carried out as described previously ${ }^{38}$ with minor modifications (for details see Supplementary Methods). Peptides were analyzed by mass spectrometry (see Supplementary Methods). For protein identification, the publicly available tobacco (N. tabacum) database from Uniprot (2013) and an in-house V. dahliae (PeterEsse_JR2_ Genome_2013) database were queried. Results were analyzed with MaxQuant (Version 1.3.0.5.) in label-free mode ${ }^{39}$. Result filtering and statistical analyses were carried out as described previously ${ }^{40}$. Tobacco $\mathrm{CHI} 1$ and $\mathrm{CHI} 2$ domain identities were confirmed using InterPro (Version 62.0; http://www.ebi.ac.uk/interpro/).

\section{Phylogenetic analysis of plant chitinases}

To discern putative chitinases closely related to the two tobacco chitinases identified by mass spectrometry, we queried the predicted proteomes of the Solanaceous plants tomato (Version 2.4), Solanum tuberosum (potato; Version 3.4), Nicotiana benthamiana (Version 0.4.4), tobacco (all from solgenomics.net), as well as A. thaliana (thale cress; TAIR Version 10) for proteins containing chitin-binding (PF00187) and glycoside hydrolase family 19 (PF00182) domains using HMMer (Version 3.1b) ${ }^{41}$ and a local Pfam database (Version 27) ${ }^{42}$. Proteins without predicted $\mathrm{N}$-terminal secretion signal and truncated proteins were removed. Remaining protein sequences were aligned using MAFFT (Version 7.271) ${ }^{35}$. Maximum likelihood phylogeny was inferred using RaXML (Version 8.2.4; WAG model of amino acid change and GAMMA model of rate heterogeneity) ${ }^{43}$, and its robustness was assessed using 1,000 bootstrap replicates.

\section{Production of recombinant tomato chitinases}

Pichia pastoris GS115 expressing recombinant tomato chitinases SIChi2, SIChi4 or SIChi13 $3^{21}$ were grown in a New Brunswick Bioflo 3000 fermenter for protein production and purification as described previously ${ }^{21,44}$ with minor modifications in the purification process (for details see Supplementary Methods). Purified chitinases were stored in $0.2 \mathrm{M}$ $\mathrm{NaCl}$ and concentrations were measured as described above. 


\section{Colorimetric chitinase activity assay}

Endo- and exochitinase activities of purified chitinases SIChi2, SIChi4, SIChi13 were tested using $0.2 \mathrm{mg} / \mathrm{mL}$ or $0.5 \mathrm{mg} / \mathrm{mL}$ 4-Nitrophenyl $\beta$-D-N, $\mathrm{N}^{\prime}, \mathrm{N}^{\prime \prime}$-triacetylchitotriose and $\mathrm{N}$-Nitrophenyl $\mathrm{N}$-acetyl- $\beta$-D-glucosaminide substrates, respectively, from the Chitinase Assay Kit (CS0980, Sigma, St. Louis, MO, USA) according to manufacturer's instructions. Effector proteins or moranoline-conjugated chitin oligomers ${ }^{28}$ were pre-incubated with $\mathrm{SIChi} 4$ at indicated concentrations for $15 \mathrm{~min}$ prior to addition to substrates. The release of $p$-nitrophenol (4-nitrophenol) was measured after 2 hours of incubation in $0.25 \mathrm{M}$ ammonium sulfate, $0.1 \mathrm{M}$ BisTris, pH 5.5 at $37^{\circ} \mathrm{C}$ in a CLARIOstar plate reader (BMG LABTECH, Ortenberg, Germany) at $405 \mathrm{~nm}$. Absorbance values were exported from the MARS data analysis software (BMG LABTECH, Ortenberg, Germany). Enzyme activities were calculated as described in the kit's technical bulletin. Statistical differences between treatments were determined by one-way ANOVA followed by Tukey's post-hoc analysis in R (Version 3.3.2).

\section{Acknowledgements}

The authors thank W. van der Linden and H. Fotowatikha Someeserai for technical assistance, and Drs. M.H.A.J. Joosten, P.J.G.M. de Wit, and D.E. Cook for critical comments to earlier versions of the manuscript. Work in the laboratory of B.P.H.J. Thomma is supported by the Research Council for Earth and Life sciences (ALW) of the Netherlands Organization for Scientific Research (NWO).

\section{Author contributions}

H.R. and B.P.H.J.T. conceived the project. H.R., J.R.M. and B.P.H.J.T. designed the experiments. H.R., J.C.B., N.C.S., C.G., J.O., S.B., and M.F.S. carried out the experiments and analyzed the data. M.O. provided crucial experimental material. H.R. and B.P.H.J.T. wrote the manuscript. All authors read and approved the final manuscript.

\section{References}

1 Rovenich, H., Boshoven, J. C. \& Thomma, B. P. H. J. Filamentous pathogen effector functions: of pathogens, hosts and microbiomes. Curr. Opin. Plant Biol. 20, 96-103, doi:10.1016/j.pbi.2014.05.001 (2014).

2 de Jonge, R. et al. Tomato immune receptor Ve1 recognizes effector of multiple fungal pathogens uncovered by genome and RNA sequencing. Proc. Natl. Acad. Sci. USA 109, 5110-5115, doi:10.1073/ pnas.1119623109 (2012). van Loon, L.C., Rep, M.\&Pieterse, C.M.J.Significance of inducible defense-related proteins in infected plants. Annu. Rev. Phytopathol. 44, 135-162, doi:10.1146/ annurev.phyto.44.070505.143425 (2006).

4 Fradin, E. F. \& Thomma, B. P. H. J. Physiology and molecular aspects of Verticillium wilt diseases caused by $V$. dahliae and $V$. albo-atrum. Mol. Plant Pathol. 7, 71-86, doi:10.1111/J.13643703.2006.00323.X (2006). 
5 Klosterman, S. J., Atallah, Z. K., Vallad, G. E. \& Subbarao, K. V. Diversity, pathogenicity, and management of Verticillium species. Annu. Rev. Phytopathol. 47, 39-62, doi:10.1146/annurevphyto-080508-081748 (2009).

6 de Wit, P. J. G. M. Apoplastic fungal effectors in historic perspective; a personal view. New Phytol., doi:10.1111/nph.14144 (2016).

7 Song, Y. et al. Tomato immune receptor Ve1 recognizes surface-exposed co-localized $\mathrm{N}$ - and C-termini of Verticillium dahliae effector Ave1. bioRxiv, doi:https://doi.org/10.1101/103473 (2017).

8 de Jonge, R. et al. Extensive chromosomal reshuffling drives evolution of virulence in an asexual pathogen. Genome Res. 23, 1271-1282, doi:10.1101/gr.152660.112 (2013).

9 Faino, L. et al. Single-molecule real-time sequencing combined with optical mapping yields completely finished fungal genome. $\mathrm{mBio}$ 6, doi:10.1128/mBio.00936-15 (2015).

10 Faino, L. et al. Transposons passively and actively contribute to evolution of the two-speed genome of a fungal pathogen. Genome Res. 26, 1091-1100, doi:10.1101/gr.204974.116 (2016).

11 Ludidi, N. N., Heazlewood, J. L., Seoighe, C., Irving, H. R. \& Gehring, C. A. Expansin-like molecules: Novel functions derived from common domains. J. Mol. Evol. 54, 587-594, doi:10.1007/s00239-0010055-4 (2002).

12 Gehring, C. A. \& Irving, H. R. Natriuretic peptides - a class of heterologous molecules in plants. Int $J$ Biochem Cell B 35, 1318-1322, doi:10.1016/S13572725(03)00032-3 (2003).

13 Toop, T. \& Donald, J. A. Comparative aspects of natriuretic peptide physiology in non-mammalian vertebrates: a review. J. Comp. Physiol. B 174, 189204, doi:10.1007/s00360-003-0408-y (2004).

14 Ruzvidzo, O., Donaldson, L., Valentine, A. \& Gehring, C. The Arabidopsis thaliana natriuretic peptide AtPNP-A is a systemic regulator of leaf dark respiration and signals via the phloem. J. Plant Physiol. 168, 1710-1714, doi:10.1016/j. jplph.2011.03.011 (2011).

15 Gottig, N. et al. Xanthomonas axonopodis pv. citri uses a plant natriuretic peptide-like protein to modify host homeostasis. Proc. Natl. Acad. Sci. USA 105, 18631-18636, doi:10.1073/pnas.0810107105 (2008).
16 Nembaware, V., Seoighe, C., Sayed, M. \& Gehring, C. A plant natriuretic peptide-like gene in the bacterial pathogen Xanthomonas axonopodis may induce hyper-hydration in the plant host: a hypothesis of molecular mimicry. BMC Evol. Biol. 4, doi:doi10.1186/1471-2148-4-10 (2004).

17 Pharmawati, M., Maryani, M. M., Nikolakopoulos, T., Gehring, C. A. \& Irving, H. R. Cyclic GMP modulates stomatal opening induced by natriuretic peptides and immunoreactive analogues. Plant Physiol. Biochem. 39, 385-394, doi:Doi 10.1016/S09819428(01)01252-9 (2001).

18 Kombrink, A., Sánchez-Vallet, A. \& Thomma, B. P. $\mathrm{H}$. J. The role of chitin detection in plant-pathogen interactions. Microb. Infect. 13, 1168-1176, doi:10.1016/j.micinf.2011.07.010 (2011).

19 Sánchez-Vallet, A., Mesters, J. R. \& Thomma, B. P. H. J. The battle for chitin recognition in plantmicrobe interactions. FEMS Microbiol. Rev. 39, 171183, doi:10.1093/femsre/fuu003 (2015).

20 Rovenich, H., Zuccaro, A. \& Thomma, B. P. H. J. Convergent evolution of filamentous microbes towards evasion of glycan-triggered immunity. New Phytol., doi:10.1111/nph.14064 (2016).

21 Karimi Jashni, M. et al. Synergistic action of a metalloprotease and a serine protease from Fusarium oxysporum f. sp. lycopersici cleaves chitin-binding tomato chitinases, reduces their antifungal activity, and enhances fungal virulence. Mol. Plant-Microbe Interact. 28, 9961008, doi:10.1094/Mpm1-04-15-0074-R (2015).

22 Iseli, B., Boller, T. \& Neuhaus, J. M. The N-terminal cysteine-rich domain of tobacco class I chitinase is essential for chitin binding but not for catalytic or antifungal activity. Plant Physiol. 103, 221-226 (1993).

23 Suarez, V. et al. Substrate specificity and antifungal activity of recombinant tobacco class I chitinases. Plant Mol. Biol. 45, 609-618 (2001).

24 Ohnuma, T. et al. Crystal structure and chitin oligosaccharide-binding mode of a 'loopful' family GH19 chitinase from rye, Secale cereale, seeds. FEBS J. 279, 3639-3651, doi:10.1111/j.17424658.2012.08723.x (2012).

25 Houterman, P. M. et al. The mixed xylem sap proteome of Fusarium oxysporum-infected tomato plants. Mol. Plant Pathol. 8, 215-221, doi:10.1111/j.1364-3703.2007.00384.x (2007). 
26 Danhash, N., Wagemakers, C. A., van Kan, J. A. \& de Wit, P. J. G. M. Molecular characterization of four chitinase cDNAs obtained from Cladosporium fulvum-infected tomato. Plant Mol. Biol. 22, 1017 1029 (1993).

27 Joosten, M. H. A. J. \& De Wit, P. J. G. M. Identification of several pathogenesis-related proteins in tomato leaves inoculated with Cladosporium fulvum (syn. Fulvia fulva) as 1,3-beta-glucanases and chitinases. Plant Physiol. 89, 945-951 (1989).

28 Ogata, M. et al. A novel transition-state analogue for lysozyme, 4-O-beta-tri-N-acetylchitotriosyl moranoline, provided evidence supporting the covalent glycosyl-enzyme intermediate. J. Biol. Chem. 288, 6072-6082, doi:10.1074/jbc. M112.439281 (2013).

29 Shinya, T., Nakagawa, T., Kaku, H. \& Shibuya, N. Chitin-mediated plant-fungal interactions: catching, hiding and handshaking. Curr. Opin. Plant Biol. 26, 64-71, doi:10.1016/j.pbi.2015.05.032 (2015).

30 Kombrink, A. et al. Verticillium dahliae LysM effectors differentially contribute to virulence on plant hosts. Mol. Plant Pathol., doi:10.1111/ mpp.12520 (2016).

31 de Jonge, R. \& Thomma, B. P. H. J. Fungal LysM effectors: extinguishers of host immunity? Trends Microbiol. 17, 151-157, doi:10.1016/j. tim.2009.01.002 (2009).

32 Kombrink, A. \& Thomma, B. P. H. J. LysM Effectors: Secreted proteins supporting fungal life. PLOS Path. 9, doi:10.1371/journal.ppat.1003769 (2013).

33 Soanes, D. \& Richards, T. A. Horizontal gene transfer in eukaryotic plant pathogens. Annu. Rev. Phytopathol. 52, 583-614, doi:10.1146/annurevphyto-102313-050127 (2014).

34 Masachis, S. et al. A fungal pathogen secretes plant alkalinizing peptides to increase infection. Nature Microbiol. 1, 16043, doi:10.1038/ nmicrobiol.2016.43 (2016)

35 Katoh, K., Misawa, K., Kuma, K. \& Miyata, T. MAFFT: a novel method for rapid multiple sequence alignment based on fast Fourier transform. Nucleic Acids Res. 30, 3059-3066 (2002).
36 Fradin, E. F. et al. Genetic dissection of Verticillium wilt resistance mediated by tomato Ve1. Plant Physiol. 150, 320-332, doi:10.1104/pp.109.136762 (2009).

37 Santhanam, P. Random insertional mutagenesis in fungal genomes to identify virulence factors. Meth. Mol. Biol. 835, 509-517, doi:10.1007/978-1 61779-501-5 31 (2012).

38 Liebrand, T. W. et al. Endoplasmic reticulumquality control chaperones facilitate the biogenesis of $\mathrm{Cf}$ receptor-like proteins involved in pathogen resistance of tomato. Plant Physiol. 159, 1819-1833, doi:10.1104/pp.112.196741 (2012).

39 Cox, J. et al. Accurate proteome-wide labelfree quantification by delayed normalization and maximal peptide ratio extraction, termed MaxLFQ. Mol. Cell. Proteomics 13, 2513-2526, doi:10.1074/mcp.M113.031591 (2014).

40 Smaczniak, C. et al. Proteomics-based identification of low-abundance signaling and regulatory protein complexes in native plant tissues. Nature Protocols 7, 2144-2158, doi:10.1038/nprot.2012.129 (2012)

41 Eddy, S. R. Accelerated Profile HMM Searches. PLoS Comput. Biol. 7, e1002195, doi:10.1371/journal. pcbi.1002195 (2011).

42 Finn, R. D. et al. The Pfam protein families database. Nucleic Acids Res. 38, D211-222, doi:10.1093/nar/ gkp985 (2010).

43 Stamatakis, A. RAxML version 8: a tool for phylogenetic analysis and post-analysis of large phylogenies. Bioinformatics 30, 1312-1313, doi:10.1093/bioinformatics/btu033 (2014)

44 Kombrink, A. Heterologous production of fungal effectors in Pichia pastoris. Meth. Mol. Biol. 835, 209-217, doi:10.1007/978-1-61779-501-5_13 (2012). 


\section{Supplementary methods}

\section{Plant materials}

Tobacco (Nicotiana tabacum cv. Petit Havana SR1) and tomato plants were grown as described previously ${ }^{1-3}$. Following agroinfiltration, tobacco plants were transferred to climate chambers with $21^{\circ} \mathrm{C} / 19^{\circ} \mathrm{C}$ during $12 / 12$ hours day/night periods and $70 \%$ relative humidity.

\section{Production and purification of recombinant effector proteins}

The sequences encoding mature VdAve1, VnAve1, and XacPNP were amplified from pGEM-T plasmid DNA carrying the respective gene ${ }^{4}$. Forward and reverse primers included Xhol and BamHI restriction sites, respectively, for restriction digest and ligation into $\mathrm{pET}$ $15 \mathrm{~b}$ with an N-terminal His tag sequence (Novagen, Madison, WI, USA) (Supplementary Table 1). The resulting expression vectors were confirmed by sequencing and used to transform E. coli strain BL21. Positive transformants were selected by colony PCR using a pFBT005 forward primer with gene specific reverse primers (Supplementary Table 1).

For heterologous protein production, BL21 cells were grown in 1xYT liquid medium at $37^{\circ} \mathrm{C}$ with constant shaking at $200 \mathrm{rpm}$. Protein production was induced with $1 \mathrm{mM}$ IPTG final concentration when cultures reached an $\mathrm{OD}_{600}=2$ to ensure maximum yields. To favor the generation of inclusion bodies, cultures were kept at $42^{\circ} \mathrm{C}, 200 \mathrm{rpm}$ for 2 hours of protein production. Cell pellets were snap-frozen in liquid nitrogen and then washed with $100 \mathrm{mM} \mathrm{NaCl}, 1 \mathrm{mM}$ EDTA, and $10 \mathrm{mM}$ Tris at $\mathrm{pH}$ 8.5. Cells were disrupted by stirring for 1 hour in lysis buffer (100 mM Tris, $150 \mathrm{mM} \mathrm{NaCl}, 10 \%$ glycerol, $6 \mathrm{mg} / \mathrm{mL}$ lysozyme (Sigma, St. Louis, MO, USA), $2 \mathrm{mg} / \mathrm{mL}$ deoxycholic acid, $0.06 \mathrm{mg} / \mathrm{mL}$ DNasel, protease inhibitor cocktail (Roche, Mannheim, Germany)) at $4^{\circ} \mathrm{C}$. Soluble and insoluble fractions were separated by centrifuging at 20,000 $\mathrm{g}$ for $10 \mathrm{~min}$. The insoluble protein pellets were washed with $10 \mathrm{~mL} 1 \mathrm{M}$ guanidine hydrochloride $(\mathrm{GnHCl}), 10 \mathrm{mM}$ Tris at $\mathrm{pH} 8.0$ and then denatured in $10 \mathrm{~mL} 6 \mathrm{M} \mathrm{GnHCl}, 10 \mathrm{mM} \beta$-mercaptoethanol, $10 \mathrm{mM}$ Tris at $\mathrm{pH}$ 8.0. Samples were incubated for 1 hour at room temperature. Non-denatured debris was pelleted by centrifuging at $20,000 \times \mathrm{g}$ for $10 \mathrm{~min}$ and discarded. Denaturation was allowed to continue for additional 3-4 hours.

Heterologously produced proteins were then purified by metal affinity chromatography using a column packed with $10 \mathrm{~mL} 50 \%$ His60 Ni ${ }^{2+}$ Superflow Resin (Clontech, Mountain View, CA, USA) and connected to a BioLogic LP System (BioRad, Veenendaal, The Netherlands). Each run was monitored with the LP Data View software (V1.03; BioRad, Veenendaal, The Netherlands). The column was equilibrated with 5-10 volumes of wash buffer $(6 \mathrm{M} \mathrm{GnHCl}, 10 \mathrm{mM}$ Tris, $20 \mathrm{mM}$ imidazole, $10 \mathrm{mM}$ reduced glutathione, $2 \mathrm{mM}$ 
oxidized glutathione, $\mathrm{pH}$ 8.0). Protein samples were loaded at a flow rate of $0.5 \mathrm{~mL} / \mathrm{min}$ to allow maximum binding of affinity-tagged proteins to the nickel resin. Contaminating proteins were removed by washing with 10-20 column volumes of washing buffer. Bound proteins were either eluted in a $50 \mathrm{~mL}$ gradient from $100 \%$ washing buffer to $100 \%$ elution buffer ( $6 \mathrm{M} \mathrm{GnHCl}, 10 \mathrm{mM}$ Tris, $200 \mathrm{mM} \mathrm{NaCl}, 500 \mathrm{mM}$ imidazole, $10 \mathrm{mM}$ reduced glutathione, $2 \mathrm{mM}$ oxidized glutathione, $\mathrm{pH}$ 8.0) or under denaturing conditions. Eluted fractions containing recombinant proteins were analyzed by PAGE separation on 4-15\% Mini-PROTEAN ${ }^{\circ}$ TGX Stain-Free ${ }^{\mathrm{TM}}$ Protein Gels (BioRad, Veenendaal, The Netherlands). Fractions containing the protein of interest were pooled. Recombinant proteins eluted in denaturation buffer were dialyzed (Spectra/Por 3 Dialysis Membrane, MWCO $=3.5$ $\mathrm{kDa}$ ) step-wise against 20 volumes of $0.25 \mathrm{M}$ ammonium sulfate, $0.1 \mathrm{M}$ BisTris, pH 5.5 with decreasing $\mathrm{GnHCl}$ concentrations for refolding. Each dialysis step was allowed to proceed for at least 2 hours. Samples were kept overnight in buffer containing $1 \mathrm{M} \mathrm{GnHCl}$ supplemented with glutathione to allow further disulphide reshuffling. For downstream applications, proteins were then dialyzed against $0.25 \mathrm{M}$ ammonium sulfate, $0.1 \mathrm{M}$ BisTris, $\mathrm{pH} 5.5$ and concentrated over Amicon Ultra Centrifugal units (MWCO=3 kDa, Merck Millipore, Cork, Ireland). Final concentrations were determined using the BioRad Protein Assay (BioRad, Veenendaal, The Netherlands).

\section{Biomass quantification}

For $V$. dahliae biomass quantification total DNA was extracted from lyophilized tomato stem sections of infected plants using a CTAB-based extraction buffer (100 mM Tris- $\mathrm{HCl}$ $\mathrm{pH}$ 8.0, $20 \mathrm{mM}$ EDTA, $2 \mathrm{M} \mathrm{NaCl}$, 3\% CTAB). DNA was precipitated with $1.5 \mathrm{M}$ ammonium acetate and absolute ethanol. Quantitative RT-PCR was carried out with the ITS1_F/StVe1_R primer pair (Supplementary Table 1). For sample calibration, the tomato Rubisco gene $(S I R U B)$ was targeted using SIRUB F and R primers (Supplementary Table 1). Quantitative RT-PCR conditions and result analyses were as described elsewhere ${ }^{5}$.

\section{VdAve1 target identification by immunopurification and mass spectrometry}

Immunopurifications were carried out as described previously ${ }^{6}$ with minor modifications. $\mathrm{N}$-terminally tagged GFP-Ave $1^{4}$ and Ve1-HA ${ }^{3}$ were produced in fully expanded leaves of three or four 5 to 6 week-old N. tabacum cv. Petit Havana SR1 plants. Total proteins were extracted using extraction buffer (EB) $(50 \mathrm{mM}$ Tris, $150 \mathrm{mM} \mathrm{NaCl}, 1 \%$ IGEPAL CA630 (NP40), protease inhibitor cocktail (Roche), pH 8.0). For immunopurification, 2 mg of total protein were incubated with $25 \mu \mathrm{L} 50 \%$ slurry of GFP-Trap ${ }^{\circ}$ A beads (Chromotek, Planegg-Martinsried, Germany) or a-HA affinity matrix (Pierce, Rockford, IL, USA) shaking for 1 hour at $4^{\circ} \mathrm{C}$. Beads were washed five times with EB. Peptides generated by tryptic onbead digestion of immunopurified proteins were analyzed using a Proxeon EASY nanoLC 
connected to a Thermo LTQ-Orbitrap XL mass spectrometer ${ }^{7}$. For protein identification, the publicly available tobacco (N. tabacum) database from Uniprot (2013) and an in-house V. dahliae (PeterEsse_JR2_Genome_2013) databases were queried. Results were analysed with MaxQuant 1.3.0.5. in label-free mode ${ }^{8}$. Result filtering and statistical analyses were carried out as described previously?

\section{Chitinase expression in infected tomato plants}

Tomato stem sections were collected from 9 plants 4, 8, and 12 days after inoculation with wild-type $V$. dahliae or water as mock treatment. Samples of three plants were pooled for each treatment and time point. Chitinase expression was analyzed using previously published primer pairs ${ }^{10}$. Quantitative RT-PCR was carried out as previously ${ }^{5}$. Expression values of infected plants were normalized to those in mock-treated tissue.

\section{Purification of recombinant tomato chitinases}

Following fermentation, cell-free supernatants were concentrated using a Vivaflow 200 crossflow device (MWCO $=5$ kDa, Sartorius, Göttingen, Germany) and purified under native conditions by metal affinity chromatography using a column packed with $10 \mathrm{~mL}$ $50 \%$ His60 $\mathrm{Ni}^{2+}$ Superflow Resin (Clontech, Mountain View, CA, USA) and connected to a BioLogic LP Sytem (BioRad, Veenendaal, The Netherlands). Each run was monitored with the LP Data View software (Version 1.03; BioRad, Veenendaal, The Netherlands). The column was equilibrated with 5-10 volumes of washing buffer $(20 \mathrm{mM}$ Tris, $200 \mathrm{mM} \mathrm{NaCl}$, $20 \mathrm{mM}$ imidazole, $\mathrm{pH}$ 8.0). After sample loading, the column was washed with 20 column volumes of washing buffer to remove contaminants. Specifically bound proteins were eluted in a $50 \mathrm{~mL}$ gradient from $100 \%$ washing buffer to $100 \%$ elution buffer $(20 \mathrm{mM}$ Tris, $200 \mathrm{mM} \mathrm{NaCl}, 500 \mathrm{mM}$ imidazole, $\mathrm{pH}$ 8.0). Fractions containing recombinant chitinase proteins were combined and dialyzed (Spectra/Por 3 Dialysis Membrane; $\mathrm{MWCO}=3.5$ $\mathrm{kDa}$ ) against 100 volumes of $200 \mathrm{mM} \mathrm{NaCl}$ overnight. Samples were concentrated and concentrations measured as described above. 


\section{References supplementary methods}

1 Fradin, E. F. et al. Interfamily transfer of tomato Ve1 mediates Verticillium resistance in Arabidopsis. Plant Physiol. 156, 2255-2265, doi:10.1104/pp.111.180067 (2011)

2 Fradin, E. F. et al. Genetic dissection of Verticillium wilt resistance mediated by tomato Ve1. Plant Physiol. 150, 320-332, doi:10.1104/pp.109.136762 (2009).

3 Fradin, E. F. et al. Functional analysis of the tomato immune receptor Ve1 through domain swaps with its non-functional homolog Ve2. PLoS ONE 9, e88208, doi:10.1371/journal.pone.0088208 (2014).

4 Song, Y. et al. Tomato immune receptor Ve1 recognizes surface-exposed co-localized N- and C-termini of Verticillium dahliae effector Ave1. bioRxiv, doi:https://doi.org/10.1101/103473 (2017).

5 de Jonge, R. et al. Tomato immune receptor Ve1 recognizes effector of multiple fungal pathogens uncovered by genome and RNA sequencing. Proc. Natl. Acad. Sci. USA 109, 5110-5115, doi:10.1073/pnas.1119623109 (2012).

6 Liebrand, T. W. et al. Endoplasmic reticulum-quality control chaperones facilitate the biogenesis of Cf receptor-like proteins involved in pathogen resistance of tomato. Plant Physiol. 159, 1819-1833, doi:10.1104/pp.112.196741 (2012).

7 Lu, J. et al. Filter-aided sample preparation with dimethyl labeling to identify and quantify milk fat globule membrane proteins. J. Proteomics 75, 34-43, doi:10.1016/j.jprot.2011.07.031 (2011).

8 Cox, J. et al. Accurate proteome-wide label-free quantification by delayed normalization and maximal peptide ratio extraction, termed MaxLFQ. Mol. Cell. Proteomics 13, 2513-2526, doi:10.1074/mcp. M113.031591 (2014).

9 Smaczniak, C. et al. Proteomics-based identification of low-abundance signaling and regulatory protein complexes in native plant tissues. Nature Protocols 7, 2144-2158, doi:10.1038/nprot.2012.129 (2012).

10 Karimi Jashni, M. et al. Synergistic action of a metalloprotease and a serine protease from Fusarium oxysporum f. sp lycopersici cleaves chitin-binding tomato chitinases, reduces their antifungal activity, and enhances fungal virulence. Mol. Plant-Microbe Interact. 28, 996-1008, doi:10.1094/Mpm1-04-15-0074-R (2015). 


\section{Supplementary data}

a

\begin{tabular}{|c|c|c|c|}
\hline VdAvel & SP & PNP & \\
\hline VnAvel & $\mathrm{SP}$ & PNP & \\
\hline FoAve1 & SP & PNP & \\
\hline CbAvel & $\mathrm{SP}$ & PNP & \\
\hline ChAvel & SP & PNP & \\
\hline XacPNP & SP & PNP & \\
\hline SIAve1 & SP & PNP & \\
\hline
\end{tabular}

b

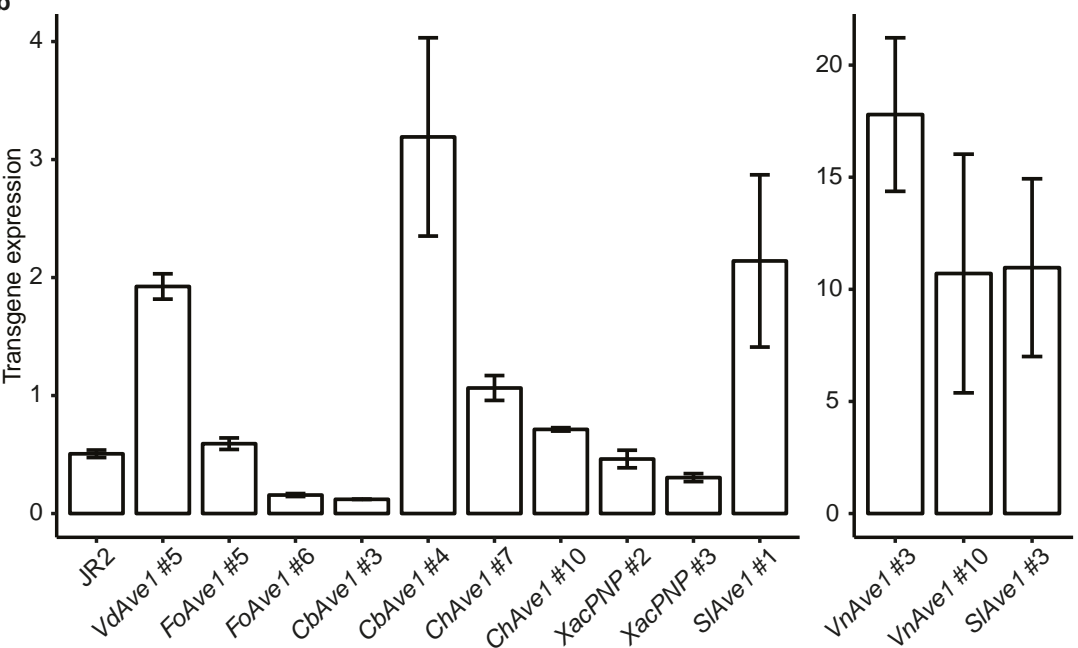

SUPPLEMENTARY FIGURE 1 | Construct design and expression of VdAve1 homologous genes from microbes and tomato in $\boldsymbol{V}$. dahliae. a, Schematic representation of VdAve1 homologs as expressed in the VdAve1 deletion mutant. The native signal peptide of the bacterial homolog XacPNP was replaced with that of VdAve 1 to ensure secretion by $V$. dahliae. b. In vitro expression of transgenes in selected strains. Following selection, transformants were grown in liquid $1 / 2$ MS medium for 5 days. Transgene expression levels were determined by qRT-PCR using transgene-specific primers and VdGAPDH for sample calibration. Bars represent mean expression values of 2-3 independently grown cultures (only CbAve1 \#3 was grown once) \pm SD. 
a

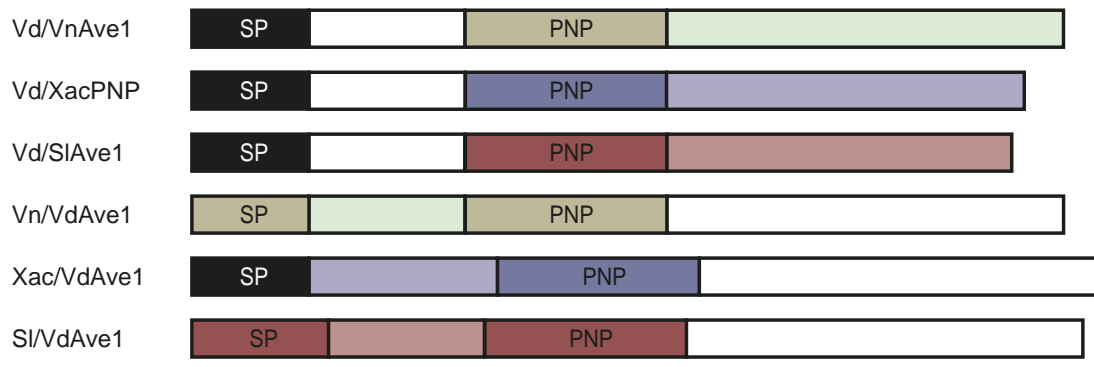

b

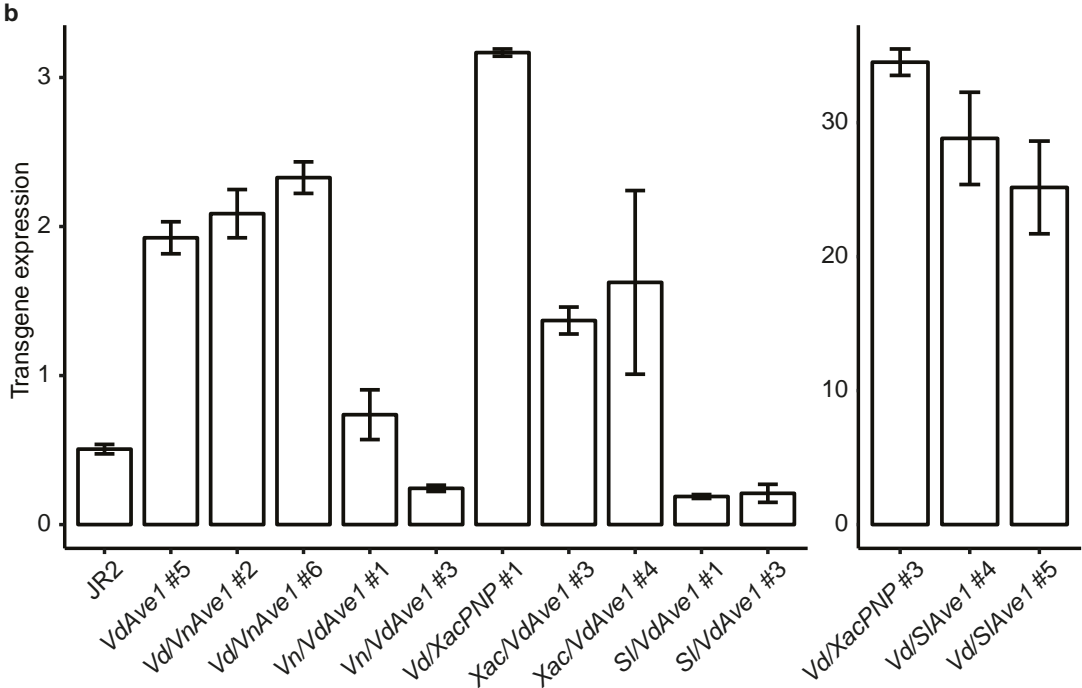

SUPPLEMENTARY FIGURE 2 | Construct design and expression of chimeric Ave1 mutants. a, Schematic representation of Ave1 chimeras as expressed in the VdAve1 deletion mutant. Black and white boxes represent domains originating from VdAve1. Colored boxes show domains taken from homologs. $\mathbf{b}$, In vitro expression of transgenes in selected strains. Transgene expression levels were determined by qRT-PCR using transgene-specific primers and VdGAPDH for sample calibration. Bars represent mean expression values of 2-3 independently grown cultures \pm SD. 


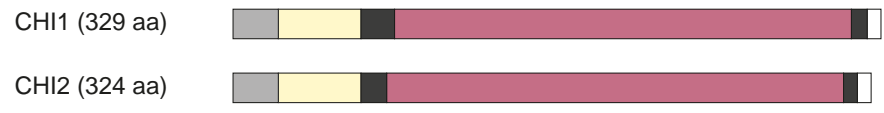

SUPPLEMENTARY FIGURE 3 | Graphic representation of tobacco chitinases. Protein structures of tobacco $\mathrm{CHI} 1$ and $\mathrm{CHI} 2$ include signal peptides (light grey), chitin-binding type-1 domains (yellow), glycosyl hydrolase 19 domains (red), and a pro-peptide at the C-terminus (white), which is removed in the mature form of each protein. 


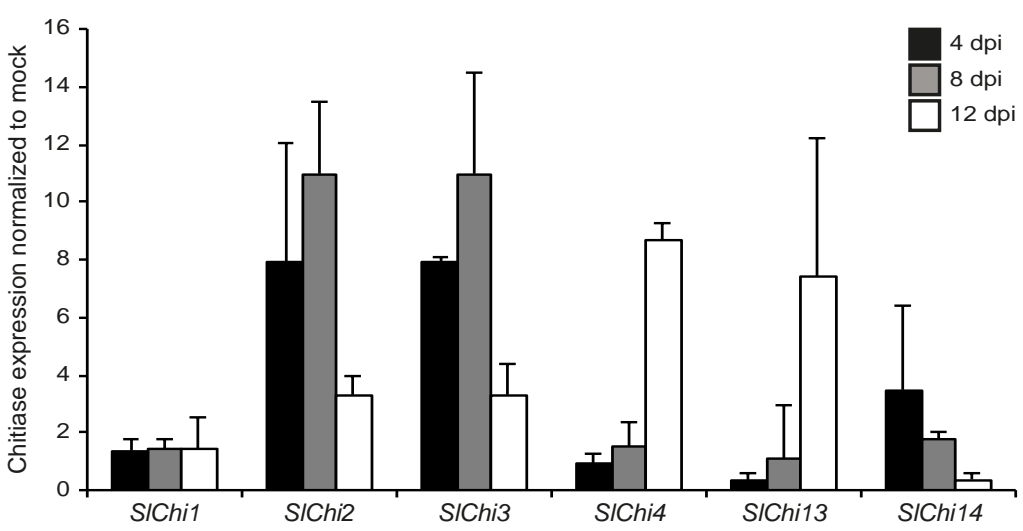

SUPPLEMENTARY FIGURE 4 | Expression of tomato endochitinase genes during $\boldsymbol{V}$. dahliae infection. Tomato stem tissue was harvested at 4,8 , and 12 days following inoculation with wild type $V$. dahliae. Endochitinase expression levels were determined by qRT-PCR with chitinase gene-specific primers and primers targeting the tomato tubulin gene for calibration. Shown are expression values relative to mock-treated tissue of three biological replicates consisting of pools of three plants from a single experiment (mean \pm SD). This experiment was performed twice. 
a

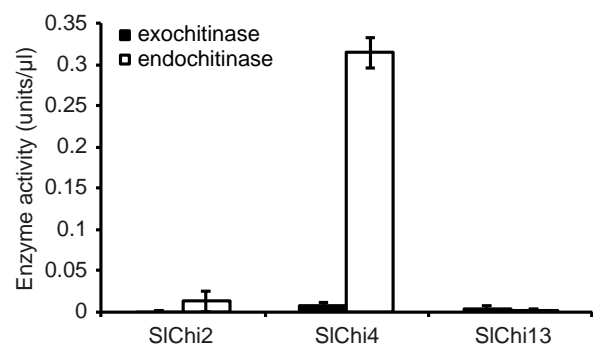

b

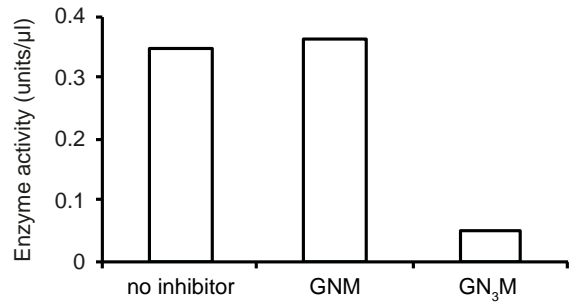

c

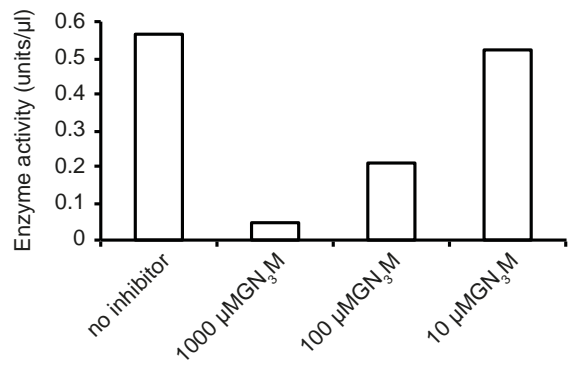

SUPPLEMENTARY FIGURE 5 | The chitinase inhibitor $\mathrm{GN}_{3} \mathrm{M}$ efficiently inhibits tomato endochitinase SIChi4. a, Comparison of chitinase activities. SIChi2, SIChi4, and SIChi13 (0.5 $\mu \mathrm{M})$ were incubated with $0.2 \mathrm{mg} / \mathrm{mL}$ substrates for $30 \mathrm{~min}$ at $37^{\circ} \mathrm{C}$. The highest amount of $p$-nitrophenol product was measured following endochitinolytic cleavage by SIChi4. Hardly any activity was detected for SIChi2 and SIChi13. Bars show mean values from two independent experiments with two technical replicates \pm SD. $\mathbf{b}$, Moranoline-coupled chitotriose $\left(\mathrm{GN}_{3} \mathrm{M}\right)$ suppresses SIChi4 activity. $\mathrm{GN}_{3} \mathrm{M}$ and GNM were added to SIChi4 at final concentrations of 1000 $\mu \mathrm{M}$. Following pre-incubation at room temperature, the mixtures were added to the substrate in reaction buffer. Enzyme activity was measured after incubation of $30 \mathrm{~min}$ at $37^{\circ} \mathrm{C}$. Data are from a single experiment. The experiment was performed twice. $c$, In order to determine whether the inhibitory effect of $\mathrm{GN}_{3} \mathrm{M}$ on $\mathrm{SIChi} 4$ is concentration-dependent, SIChi4 was pre-incubated with serial dilutions of $\mathrm{GN}_{3} \mathrm{M}$. Chitinase activity was determined as before. Data are from a single experiment. The experiment was performed twice. 
SUPPLEMENTARY TABLE 1 | Primers used in this study.

\begin{tabular}{|c|c|c|}
\hline Name & Sequence $\left(5^{\prime} \rightarrow 3^{\prime}\right)$ & Application \\
\hline Xhol_VdAve1_F & CGGTATCTCGAGGATCTAGGGACCGCATCCTAC & Protein production \\
\hline VdAve1_BamHI_R & R CGTCTAGGATCCTCATTATTATATCTGTCTAAATTCGATGTTGACC & Protein production \\
\hline Xhol_VnAve1_F & CGGTATCTCGAGCAATTAGGGACCGCATCC & Protein production \\
\hline VnAve1_BamHI_R & 2 CGTCTAGGATCCTCATTATTATATCTGTTCAAACTCG & Protein production \\
\hline Xhol_XacPNP_F & CGGTATCTCGAGGACATCGGTACAATTAG & Protein production \\
\hline XacPNP_BamHI_R & RCGTCTAGGATCCTCATTATTAAATATTTGCCCAGGG & Protein production \\
\hline pFBT005_F & GCCAGACCAATACAACAAGCA & Colony PCR and sequencing \\
\hline pFBT005_R & TCGAGATCCTGAACACCATTT & Sequencing \\
\hline VdAve1_qPCR_F & TGTTACCAAAGCAGCACACAAGG & Real-time PCR \\
\hline VdAve1_qPCR_R & СCTTATGCCTCGTTCCCTTCCAC & Real-time and colony PCR \\
\hline VnAve1_qPCR_F & GAGCCCAGACAGCTGTCAC & Real-time PCR \\
\hline VnAve1_qPCR_R & TTCAAACTCGATGTTGACCTTCT & Real-time and colony PCR \\
\hline FoAve1_qPCR_F & ATATCGGAACTGCAAATATTCTCAAC & Real-time PCR \\
\hline FoAve1_qPCR_R & CTTATACATTTCATCGTATACAGTCTGC & Real-time PCR \\
\hline CbAve1_qPCR_F & ATTCCCTTCAGGCAACCTCT & Real-time PCR \\
\hline CbAve1_qPCR_R & CGGACAAGCTTCGCAATAAT & Real-time PCR \\
\hline ChAve1_qPCR_F & CAAGATGCTATGGCAACAATATGAAC & Real-time PCR \\
\hline ChAve1_qPCR_F & GTCTTGAGGAAAATCTATCGTATTTCTG & Real-time PCR \\
\hline XacPNP_qPCR_F & GCAATCGGTTTGCTCTTTTTC & Real-time PCR \\
\hline XacPNP_qPCR_R & AGCACCGTTATCCCACAGAC & Real-time and colony PCR \\
\hline SIAve1_qPCR_F & CGTCGGGGAATCTATTTGTG & Real-time PCR \\
\hline SIAve1_qPCR_R & AAAGCATCCGTTGACAAAGC & Real-time PCR \\
\hline VdGAPDH_F & CGAGTCCACTGGTGTCTTCA & Real-time and colony PCR \\
\hline VdGAPDH_R & CCCTCAACGATGGTGAACTT & Real-time and colony PCR \\
\hline ITS1-F & AAAGTTTTAATGGTTCGCTAAGA & Real-time PCR \\
\hline St-Ve1-R & CTTGGTCATTTAGAGGAAGTAA & Real-time PCR \\
\hline SIRUB_F & GAACAGTTTCTCACTGTTGAC & Real-time PCR \\
\hline SIRUB_R & CGTGAGAACCATAAGTCACC & Real-time PCR \\
\hline SITUB_F & AACCTCCATTCAGGAGATGTTT & Real-time PCR \\
\hline SITUB_R & TCTGCTGTAGCATCCTGGTATT & Real-time PCR \\
\hline SIChi_F(TRV) & GGGGACAAGTTTGTACAAAAAAGCAGGCTTCCTTGCCCAAACTTCCCA & VIGS \\
\hline SIChi_R(TRV) & GGGGACCACTTTGTACAAGAAAGCTGGGTACTGCCGTGACCACATTCCA & VIGS \\
\hline SIChi2_F & ATGAGGCTTTCTGAATTCAC & colP \\
\hline SIChi2-HA_R & CTAAGCGTAGTCTGGGACGTCGTATGGGTACATAATATCAACTAATAGTC & colP \\
\hline SIChi4_F & ATGAGGCATTTTGAATTCATAG & colP \\
\hline SIChi4-HA_R & CTAAGCGTAGTCTGGGACGTCGTATGGGTACATAGTATCGACTAAGAGTCCG & colP \\
\hline attB1-SIChi2_F & GGGGACAAGTTTGTACAAAAAAGCAGGCTTCATGAGGCTTTCTGAATTCAC & colP \\
\hline attB1-SIChi4_F & GGGGACAAGTTTGTACAAAAAAGCAGGCTTCATGAGGCATTTTGAATTCATAG & colP \\
\hline HA-attB2_R & GGGGACCACTTTGTACAAGAAAGCTGGGTCTAAGCGTAGTCTGGGACG & colP \\
\hline
\end{tabular}




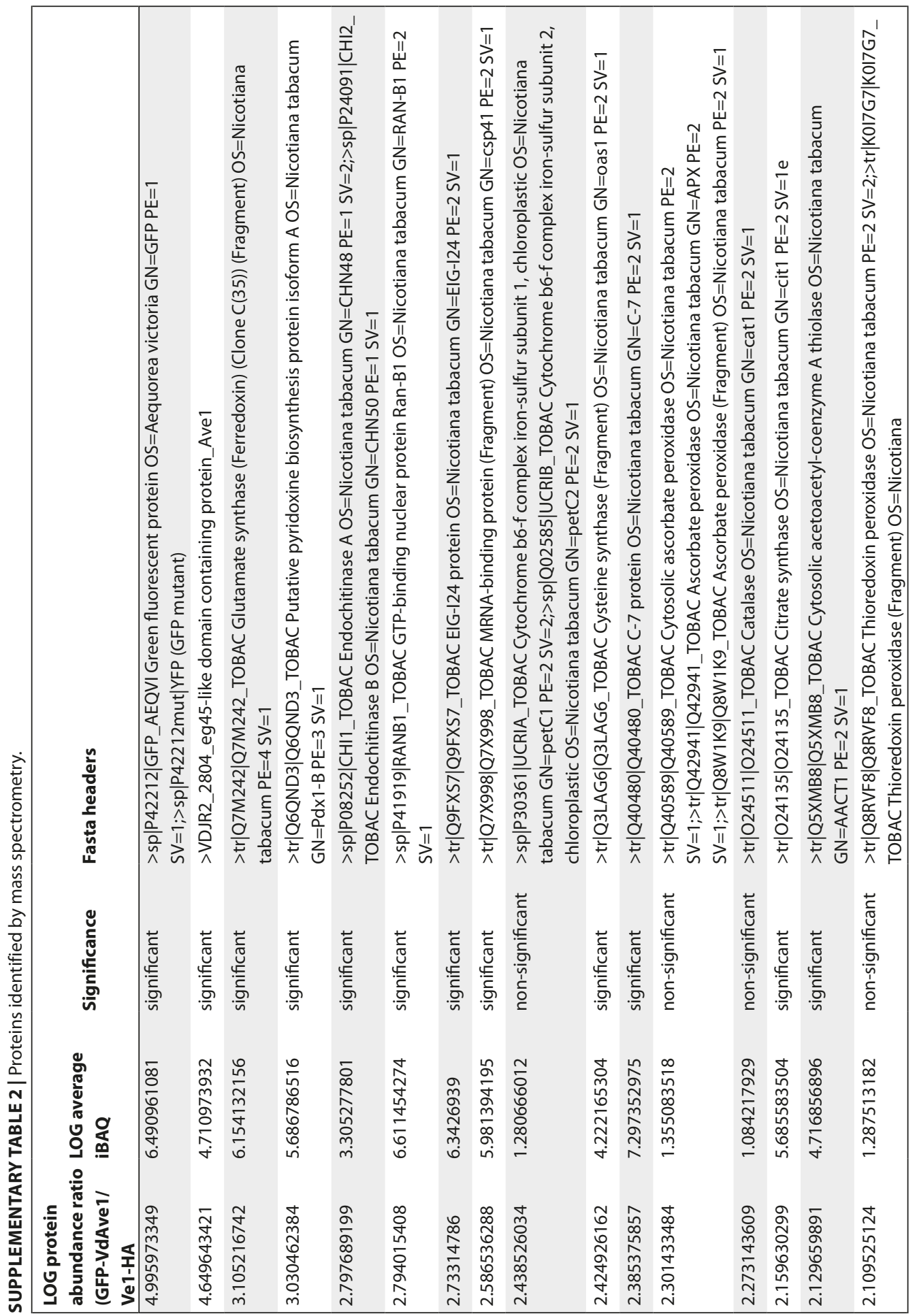




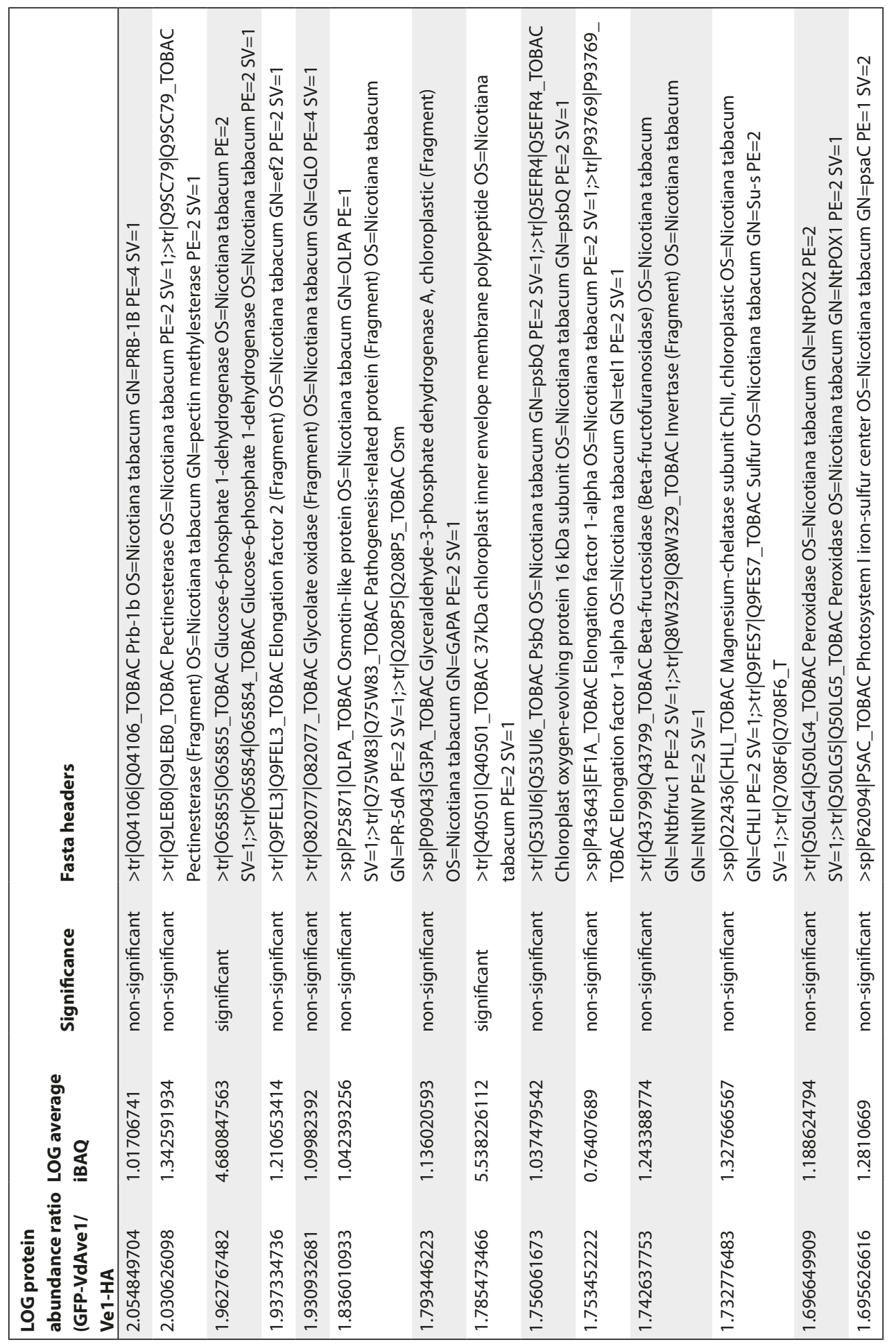




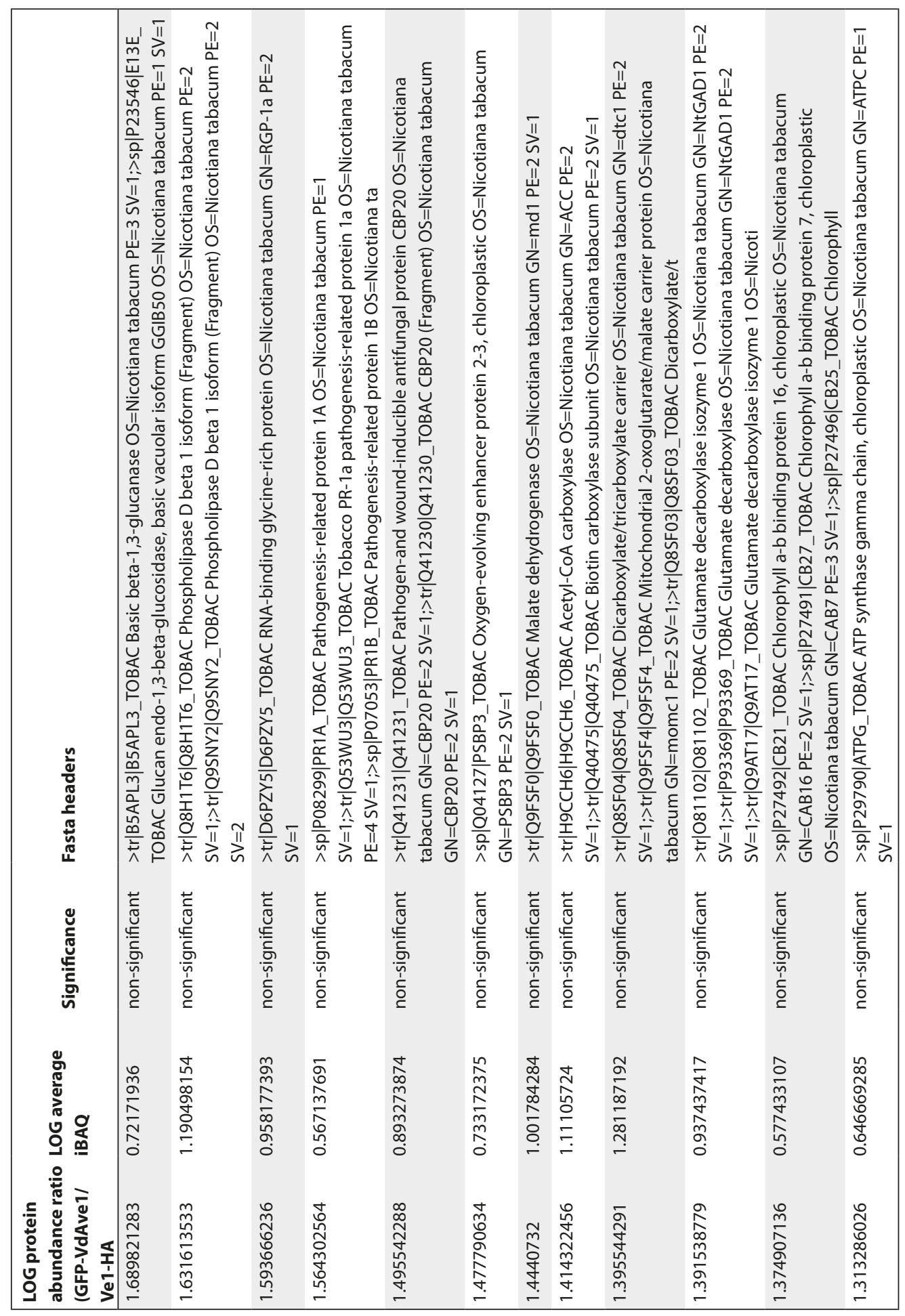




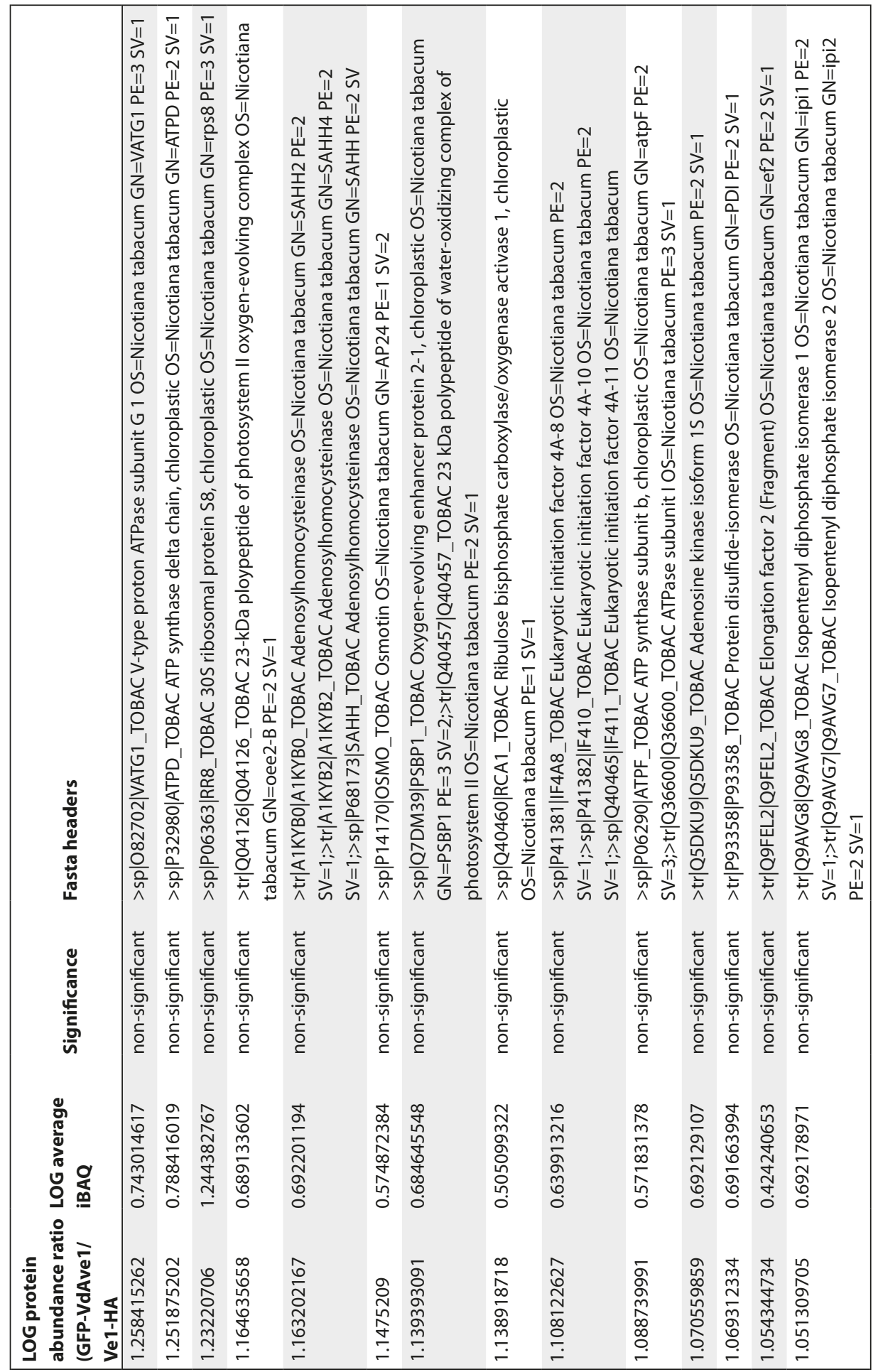




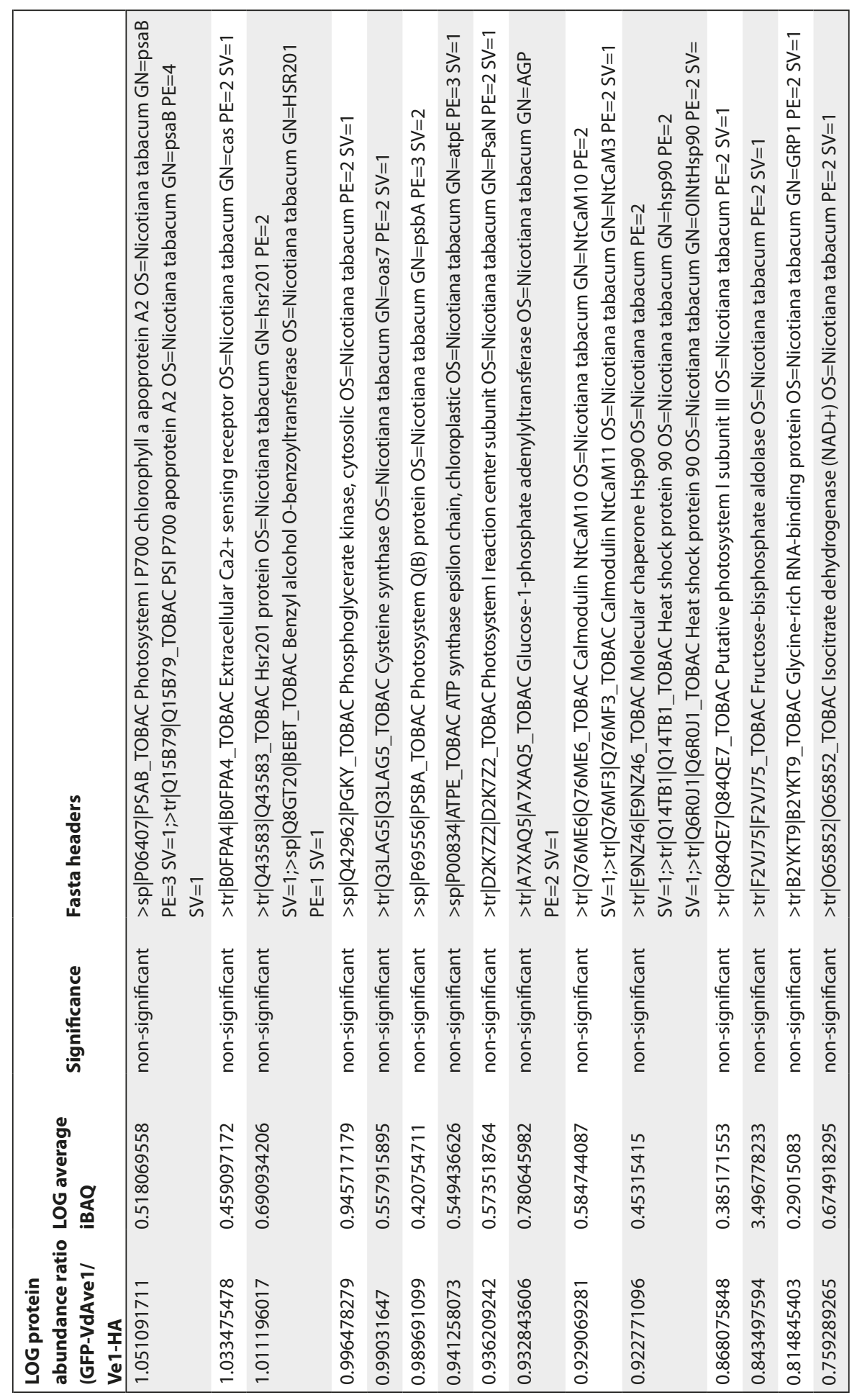




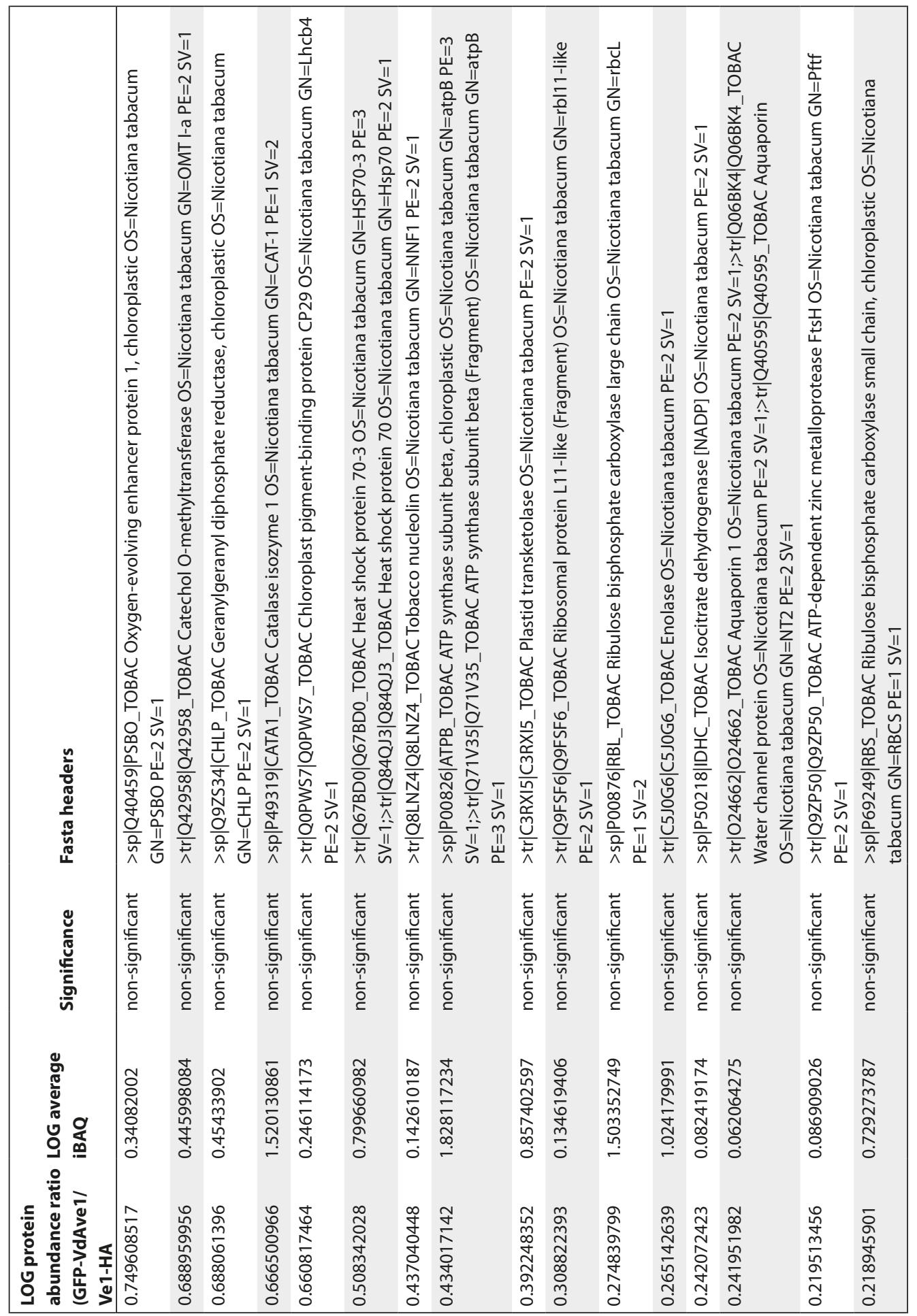




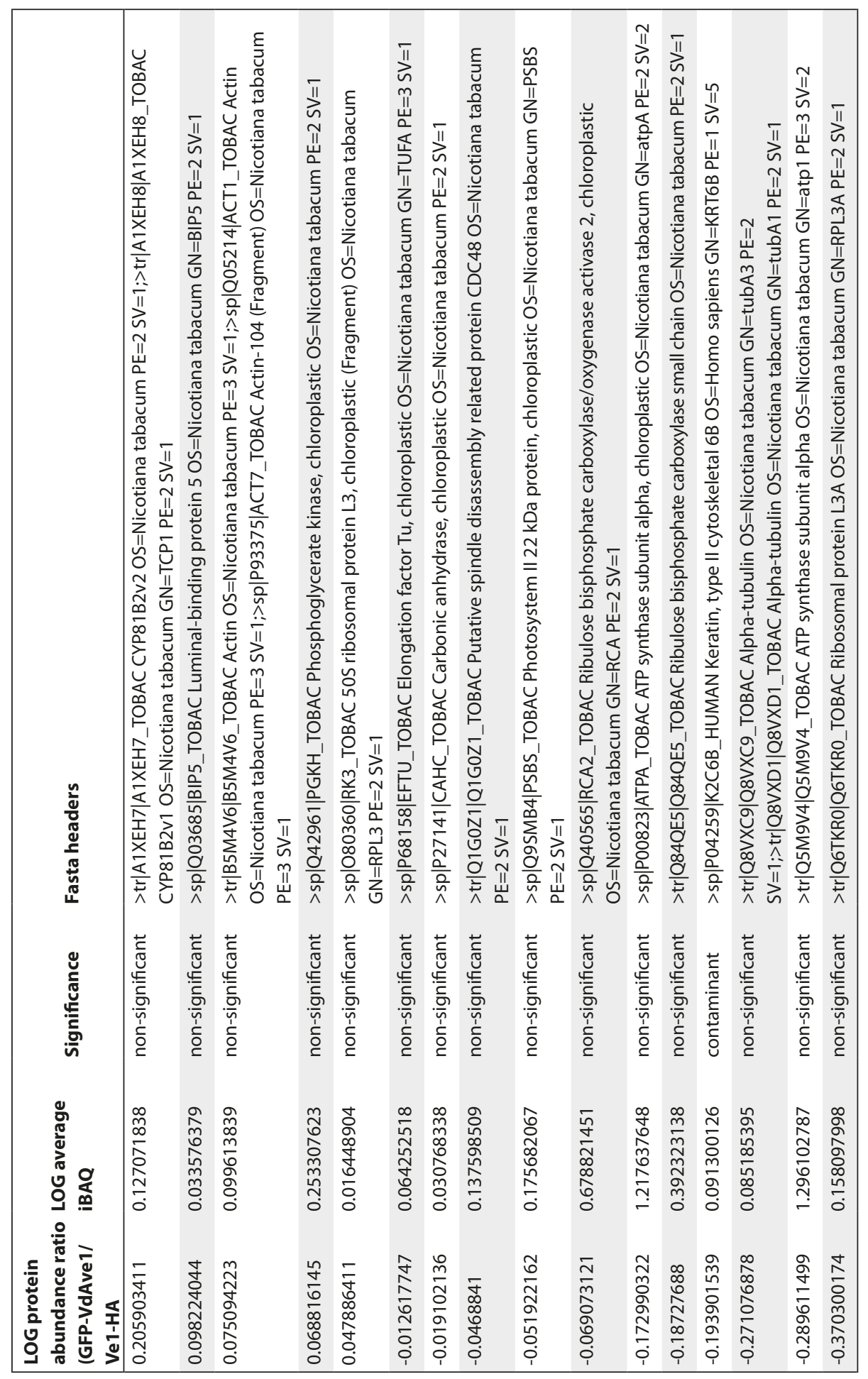




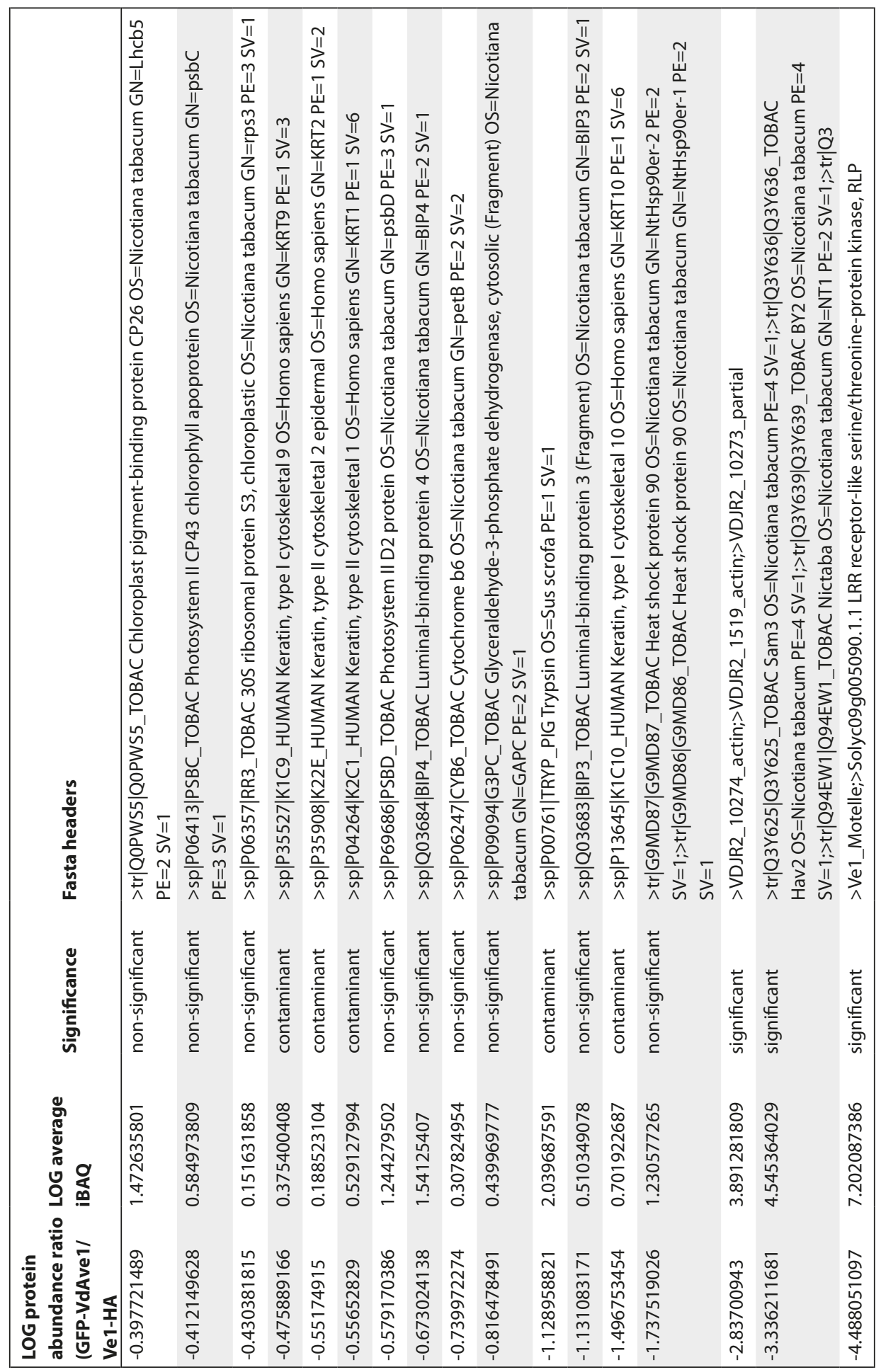




\title{
Chapter 6
}

\begin{abstract}
The tomato immune receptor $\mathrm{Ve} 1$ binds the Verticillium dahliae effector protein VdAve 1 to recruit downstream signaling components
\end{abstract}

Hanna Rovenich and Bart P.H.J. Thomma

Laboratory of Phytopathology, Wageningen University and Research, Wageningen, The Netherlands 


\begin{abstract}
Receptor proteins with extracellular leucine-rich repeats (eLRR) are crucial components of the plant immune system. In tomato, the eLRR receptor protein Ve1 governs resistance against the vascular wilt pathogen Verticillium dahliae following recognition of the effector protein Ave1 (VdAve1). In contrast, no function could be ascribed to its homolog Ve2. Analyses of domain swap mutants between Ve1 and Ve2 demonstrated that the $\mathrm{N}$-terminal eLRRs (the C1 domain) of Ve1 and Ve2 are functionally interchangeable. As the $\mathrm{C} 1$ domain has been shown to confer ligand specificity in other eLRR receptors, it has been hypothesized that it similarly mediates VdAve1 ligand perception. Here we show that both Ve1 and Ve2 interact with the VdAve1 effector. Deletion of four consecutive eLRR repeats that show the least amino acid difference between Ve1 and Ve2, does not result in the loss of binding to VdAve1. Thus, we hypothesize that this interaction is mediated by multiple clusters of eLRRs that are scattered along the $\mathrm{C} 1$ domain. Moreover, we confirm that Ve1 forms a ligand-induced receptor complex with the key regulatory eLRR receptor kinase BAK1 to initiate immune signaling, and discuss possible reasons for the lack of Ve2 functionality.
\end{abstract}




\section{Introduction}

Cell-surface receptors are essential components of plant surveillance systems detecting microbial invaders ${ }^{1}$. Recognition of appropriate ligands, which may be microbe-derived or result from microbe-mediated host manipulation, leads to the activation of immune responses to ward off potentially harmful microbes ${ }^{2}$. In turn, symbiotic microbes, including pathogens, endophytes as well as mutualists, secrete effector molecules that deregulate immune responses to successfully establish within their host ${ }^{3,4}$.

In tomato, resistance to race 1 strains of the vascular wilt pathogen Verticillium dahliae is governed by the Ve locus, which encodes the two highly identical extracellular leucine-rich repeat receptor proteins (eLRR-RPs) Ve1 and Ve2 ${ }^{5,6}$. Their extracellular domains consist of two eLRR regions ( $C 1$ and $\mathrm{C} 3$ ), which are separated by a non-eLRR island domain referred to as $\mathrm{C} 2$, and are anchored to the plasma membrane by a single-pass transmembrane domain. In contrast to eLRR-containing receptor kinases (eLRR-RKs), eLRR-RPs, including Ve1 and Ve2, carry cytoplasmic tails that lack obvious signaling domains ${ }^{7}$. Despite their identity, Ve1, but not Ve2, confers resistance to race 1 strains of $V$. dahliae in tomato and Arabidopsis thaliana (hereafter referred to as Arabidopsis) ${ }^{8,9}$. Additional homologs of $V e 1$ have been described to act as immune receptors in other plant species such as potato, wild eggplant, tobacco and hop, indicating that $\mathrm{Ve} 1$ is of ancient origin ${ }^{10}$.

By using a comparative population genomics approach, the protein that is recognized by Ve1 was identified as V. dahliae Ave1 (VdAve1, for Avirulence on $\underline{\text { Ve1 tomato) }}{ }^{11}$. During colonization of susceptible plant genotypes, $V d A v e 1$ greatly contributes to fungal virulence ${ }^{11}$. Transient Agrobacterium tumefaciens-mediated co-expression of Ve1 and VdAve1 in tobacco (Nicotiana tabacum) leaves resulted in a hypersensitive response (HR), a form of localized cell death indicative of ligand recognition by its corresponding plant immune receptor ${ }^{11,12}$. Similarly, transient potato virus X (PVX)-mediated expression of VdAve1 induced HR in tomato carrying $V e 1^{11}$. However, while the signaling cascade required for Ve1-mediated resistance is conserved in Arabidopsis, it does not involve an HR, suggesting that HR is not required for Verticillium wilt resistance ${ }^{13}$.

Many eLRR-containing receptors recognize proteinaceous ligands and the specificity with which a ligand is recognized is usually determined by the eLRR domains ${ }^{7,14-17}$. Through domain swaps between Ve1 and Ve2, and targeted mutagenesis of Ve1, we have identified three separate clusters of Ve 1 eLRR domains that are required for its function ${ }^{18,19}$.Two of these, namely eLRR1-8 and eLRR20-23, belong to the C1 domain and have been hypothesized to be involved in VdAve1 ligand binding ${ }^{19}$. In contrast, the third cluster that consists of eLRR3237 was proposed to function in immune signaling activation ${ }^{19}$.

Similar to other LRR-RPs, Ve1 and Ve2 constitutively associate with the eLRR-RK SUPPRESSOR OF BIR1-1 (SOBIR1) to form bimolecular equivalents of genuine eLRR-RKs ${ }^{18,20-22}$. Upon ligand perception, both eLRR-RKs and bimolecular eLRR-RP complexes initiate tightly 
regulated cytoplasmic signal transduction cascades ${ }^{1,23}$. Recruitment of the regulatory BRI-1 ASSOCIATED RECEPTOR KINASE (BAK1; also known as SOMATIC EMBRYOGENESIS RECEPTOR KINASE 3a (SERK3a)) and other members of the SERK family only occurs upon ligand perception and is required for eLRR-RP-mediated immunity, as was recently shown for $\mathrm{Cf}-4^{24}$ and RLP23 ${ }^{25}$. Likewise, SOBIR1 and BAK1 associate with, or are required for, immunity mediated by additional eLRR-RPs ${ }^{22,26-30}$. Interestingly, BAK1 also appears to have a negative regulatory role in eLRR-RP-mediated immunity as it was reported to attenuate xylanase-induced Eix2 immune activation in collaboration with the decoy receptor Eix ${ }^{31}$. Genetic evidence also supports a regulatory role of BAK1 in Ve1-mediated resistance ${ }^{6,9}$. In this study, we further investigated the molecular mechanisms underlying Ve1 immune complex activation following VdAve1 ligand perception using a biochemical approach. In addition, based on previous findings and those presented here, we discuss the possibilities that explain non-functionality of Ve2 in Verticillium wilt resistance.

\section{Results}

\section{Ve1 and Ve2 bind the effector protein VdAve1}

Ve1 mediates resistance to $V$. dahliae race 1 strains upon perception of the VdAve1 effector protein ${ }^{11}$. To test whether Ve1 binds VdAve1, we first co-expressed GFP-tagged VdAve1 with untagged Ve1 in Nicotiana tabacum leaves to confirm its recognition (Suppl. Fig. 1a) ${ }^{32}$. As expected, co-expression of GFP-VdAve1 with Ve1 resulted in a hypersensitive response (HR) at 6 days post infiltration (dpi) (Suppl. Fig. 1b). This HR was comparable to the response observed when untagged Ave1 was co-expressed with Ve $1^{11}$. Moreover, expression of GFPAve1 or Ave1 alone did not result in an HR (Suppl. Fig. 1b). To confirm the stability of GFPAve1 protein, we performed immunoprecipitation at 1,2,3 and 4 days following its transient expression in N. benthamiana and $N$. tabacum leaves. In both plant species the protein was stably produced at 1-2 dpi (Suppl. Fig. 1c). Due to the comparable ease of infiltration, we performed the following assays in $\mathrm{N}$. benthamiana. Co-immunoprecipitation experiments were carried out following the transient production of affinity-tagged versions of $\mathrm{Ve} 1, \mathrm{Ve} 2$ and VdAve 1 proteins at $2 \mathrm{dpi}$. The eLRR-RPs Cf9 from tomato and RLP30 from Arabidopsis served as negative controls. As expected, affinity-purification of $\mathrm{Ve} 1-\mathrm{HA}_{3}$ resulted in copurification of a protein of $39 \mathrm{kDa}$, which corresponds to the size of monomeric GFP-VdAve1 (Fig. 1a). A second, higher band was observed that could correspond to GFP-VdAve1 dimers, suggesting that effector dimers bind to Ve1. As expected based on the previously performed domain swap experiments, we similarly observed binding of monomeric and dimeric GFPVdAve1 to Ve2-HA (Fig. 1a). Importantly, GFP-VdAve1 did not co-purify with RLP30-HA 3 or Cf9- $\mathrm{HA}_{3^{\prime}}$, confirming that the interaction of VdAve1 with Ve1 and Ve2 is specific (Fig. 1a). 


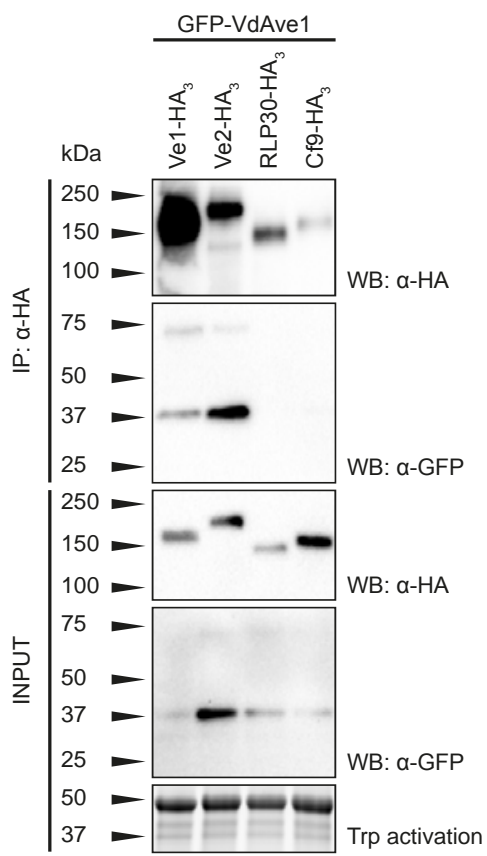

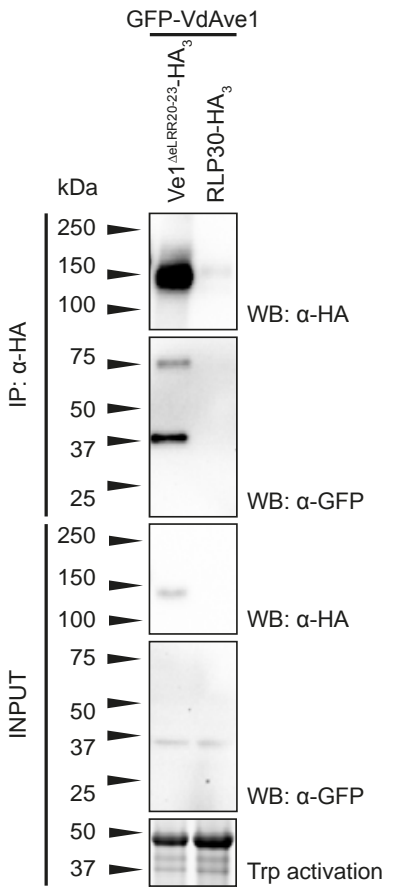

FIGURE 1 | Ve1 and Ve2 bind the fungal effector protein VdAve1. Affinity-tagged Verticillium dahliae Ave1 (VdAve1) was transiently co-expressed in Nicotiana benthamiana leaves with HA-tagged Ve1, Ve2, RLP30 and Cf9 (a) or Ve ${ }^{\triangle L R R 20-23}$ and RLP30 (b). Leaf tissue was harvested at 2 dpi for total protein extraction (INPUT). Transiently expressed receptors were immunopurified with Anti-HA magnetic beads and co-purifying GFP-VdAve1 was visualized on Western blots (WB). Equal loading of protein samples onto TGX Stain-Free ${ }^{\mathrm{TM}}$ gels was determined by tryptophan (Trp) activation under UV light. The experiments were repeated twice.

\section{eLRRs20-23 of Ve1 are not crucial for VdAve1 binding}

Ve1 and Ve2 are share 84\% amino acid identity, with the region between the eLRRs 19 and 23 being most highly conserved ${ }^{5,18}$. In a recent mutational screen, eLRR20 through eLRR23 were shown to be involved in VdAve1 recognition, since alanine substitutions of surface-exposed amino acid residues in any of the four eLRRs resulted in the loss of HR upon co-expression with VdAve1 in N. tabacum ${ }^{19}$. Because both Ve1 and Ve2 bind VdAve1, we generated a mutant construct of Ve1 lacking eLRR20-23 to test whether this highly conserved part of the extracellular region is required for effector binding. Interestingly, as with wild type Ve1, VdAve1 also co-purified with HA-tagged Ve1 ${ }^{\triangle \mathrm{ELRR} 20-23}$, whereas no binding to RLP30-HA 3 could be observed (Fig. 1b).

\section{VdAve1 homologs interact with Ve1}

VdAve1 homologs that occur in several phytopathogens are differentially perceived by tomato $\mathrm{Ve}^{32,33}$. Thus, we hypothesized that the homologs recognized by Ve1 bind to 
the receptor similar to VdAve1. We tested the interaction between the receptors and the VdAve1 homologs of V. nubilum (VnAve1), Fusarium oxysporum (FoAve1), Cercospora beticola (CbAve1), Colletotrichum higginsianum (ChAve1), and Xanthomonas citri subsp. citri (XacPNP) following their transient production in $N$. benthamiana leaf tissue. Like VdAve1, all GFP-tagged monomers and multimers of the VdAve1 homologs co-purified

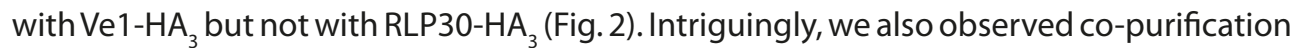
of VnAve1 and FoAve1 in Ve2-HA 3 affinity purifications whereas CbAve1, ChAve1 and XacPNP did not co-purify with Ve2 (Fig. 2).

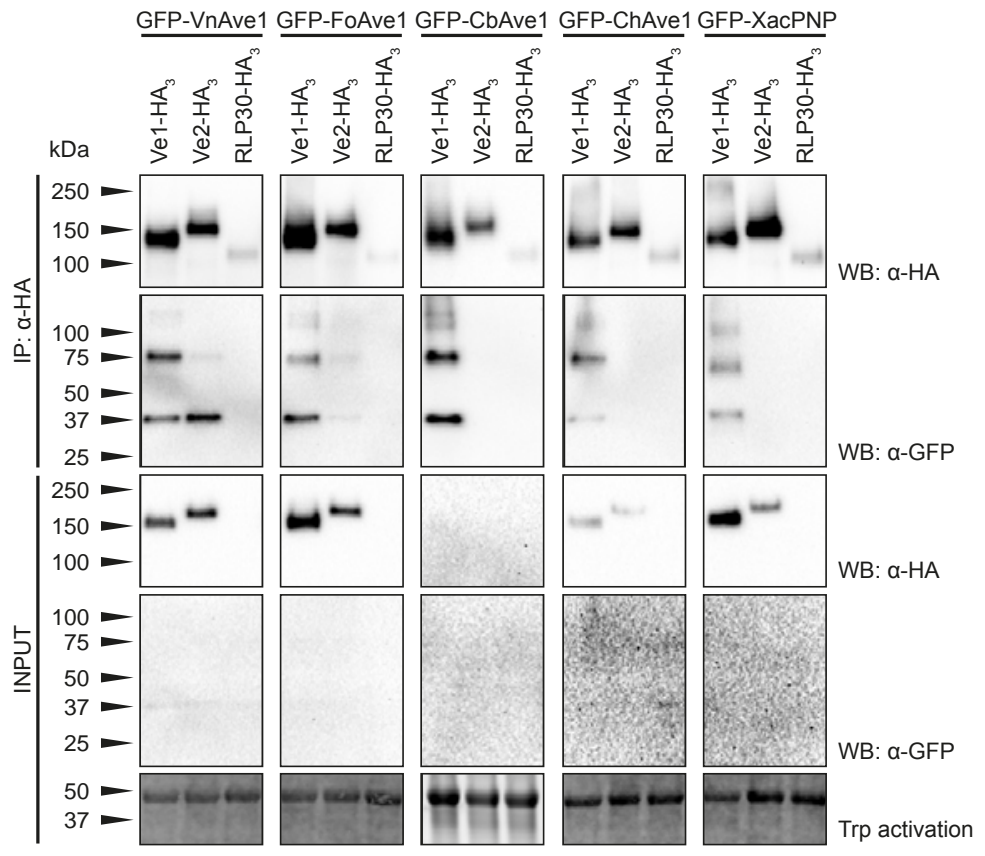

FIGURE 2 | Ve1 binds microbial Ave1 homologs. GFP-tagged VdAve1 homologs from Verticillium nubilum (Vn), Fusarium oxysporum (Fo), Cercospora beticola (Cb), Colletotrichum higginsianum (Ch), and Xanthomonas citri subsp. citri (Xac) were transiently co-expressed with $\mathrm{Ve} 1-\mathrm{HA}_{3}$ and $\mathrm{Ve} 2-\mathrm{HA}_{3}$ tomato receptor proteins in Nicotiana benthamiana leaves. Co-expression with RLP30-HA was used as negative control. Receptor proteins were purified with Anti-HA magnetic beads from total leaf extracts (INPUT) at $2 \mathrm{dpi}$. Co-purifying effectors were visualized on Western blots (WB) and total proteins were visualized by tryptophan (Trp) activation under UV light to test equal loading. The experiment was repeated twice.

\section{Ve1 recruits BAK1 upon VdAve1 ligand perception}

Ve1 constitutively interacts with the eLRR-RK SOBIR $1^{18}$ and previous genetic analysis revealed that Ve1-mediated resistance depends on $B A K 1^{9}$. Thus, we tested whether the Ve1-SOBIR1 complex recruits BAK1 upon ligand perception. To this end, Ve1-eGFP was transiently expressed together with SISOBIR1-HA and S/BAK1-cMyc in N. benthamiana leaves. Two days later, leaves were either infiltrated with GST-tagged VdAve1 or GST alone. Preliminary results show that S/BAK1-cMyc specifically co-purifies with Ve1-eGFP 
after elicitation with GST-VdAve1 but not GST (Fig. 3). This demonstrates that Ve1 forms a ligand-dependent receptor complex with BAK1, similar to Cf-4 and RLP3024,25. In contrast, SISOBIR1-HA was detected upon immunopurification of Ve1-eGFP following infiltration with both GST and GST-VdAve1 (Fig. 3), confirming the ligand-independent interaction between SOBIR1 and LRR-RPs ${ }^{18,22}$.

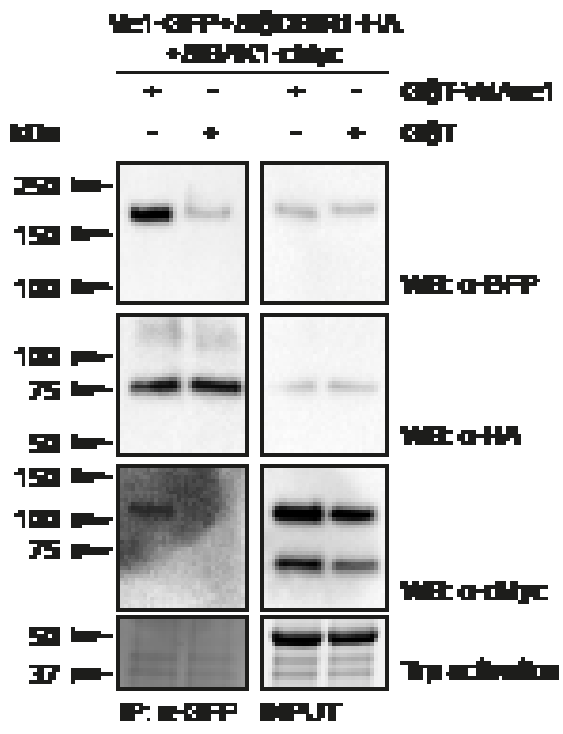

FIGURE 3 | Ve1 recruits BAK1 in a liganddependent manner. Ve1-eGFP, SISOBIR1-HA and S/BAK1-CMyc were transiently co-expressed in Nicotiana benthamiana leaves. At 2 dpi, $10 \mu \mathrm{M}$ purified GST-VdAve1 or GST were infiltrated into transformed leaves. Total protein was extracted after $30 \mathrm{~min}$ of incubation followed by a-GFP affinity purification. Co-purifying proteins were visualized on Western blots (WB). Equal loading of protein samples onto TGX Stain-Free ${ }^{\mathrm{TM}}$ gels was determined by tryptophan (Trp) activation under UV light. The experiment was done once.

\section{Discussion}

Plant eLRR-carrying surface-localized receptor proteins play crucial roles in development and immunity. While the ligands of many eLRR receptors have been identified, evidence for the physical receptor-ligand interaction is often still lacking. Here we show that the eLRR-RP Ve1 and its non-functional homolog Ve2 from tomato bind the $V$. dahliae effector protein VdAve1 (Fig. 1a). This confirms the earlier hypothesis that Ve2, like Ve1, would be able to interact with VdAve1 since the C1 domains of Ve1 and Ve2 are functionally interchangeable ${ }^{18}$. Similarly, functional dissection of the tomato $\mathrm{Cf}$ receptors $\mathrm{Cf}-4$ and Cf-9, eLRR-RPs that provide resistance to the leaf mold fungus Cladosporium fulvum, by domain swap analyses showed that their eLRRs13-16 and eLRRs10-16 contribute to ligand specificity, respectively ${ }^{17,34}$. More recently, expression of chimeric mutants of tomato Eix2 carrying the eLRR region of the eLRR-RP ReMAX resulted in responsiveness to the ReMAX ligand eMAX in N. benthamiana, which lacks an endogenous perception system for this ligand $^{28}$. In addition, sequential domain swaps between the eLRR-RKs EFR and FLS2 highlighted the importance of the EFR eLRRs for the perception of its ligand EF-Tu ${ }^{35}$. These findings demonstrate that the eLRR regions of eLRR-carrying receptors determine ligand specificity. 
While some receptors were shown to bind their ligands directly ${ }^{25,35-37}$, others perceive their ligands indirectly by monitoring plant molecules that are targeted by effector proteins. This is the case for tomato Cf-2, which does not directly interact with the C.fulvum effector Avr2 but binds tomato papain-like cysteine proteases that are inhibited by Avr2 ${ }^{38-40}$. Similarly, Cf-9 does not directly bind C. fulvum Avr9 but requires a high-affinity binding site ${ }^{41,42}$. In addition, many intracellular nucleotide-binding domain leucine-rich repeat receptors (NLRs) recognize pathogen effectors indirectly ${ }^{43}$. This is the case for the Arabidopsis NBLRR receptors RPM1 and RPS2, both of which recognize modifications of the RIN4 protein by the Pseudomonas syringae effectors AvrB or AvrRPM1 and AvrRpt2, respectively ${ }^{44-46}$. Whether or not the interaction of Ve1 and Ve2 with VdAve1 is direct or indirect cannot be concluded from our experiments, and still needs to be confirmed. After all, the plant species that were used for the protein interaction studies may share the effector target.

Phylogenetic analysis of VdAve1 resulted in the identification of homologs in the fungal pathogens F. oxysporum, C. higginsianum, and C. beticola, the saprotroph V. nubilum, as well as in the biotrophic bacterial pathogen Xanthomonas citri subsp. citri ${ }^{11}$. Strikingly, however, most homologs were found in plants, with the most closely related homologs present in tomato (Solanum lycopersicum; SIAve1) and grape (Vitis vinifera; VvPNP) ${ }^{11}$. Co-expression of these homologs with Ve1 in $N$. tabacum was previously shown to result in distinct patterns of HR, corresponding to their increasing divergence from VdAve ${ }^{32}$. Interestingly, however, despite the differences in recognition by Ve1, all microbial homologs bind the tomato receptor, similar to VdAve1 (Fig. 2). This suggests that the binding properties are conserved between VdAve1 and its homologs but that the physical receptor-ligand interaction is not sufficient for the activation of Ve1-mediated immunity. Potentially, this could be attributed to differential strengths of the interactions, which may be supported by the finding that VnAve1 and FoAve1 co-purify with Ve2-HA, whereas CbAve1, ChAve1 and XacPNP do not.

The region composed of eLRR20-23 displays very few amino acid differences between Ve1 and Ve2, and has previously been shown to be required for VdAve1 recognition and Ve1-mediated resistance to $V$. dahliae ${ }^{19}$. Since both receptors interact with the VdAve1 ligand, we tested whether eLRR20-23 are required for VdAve1 binding. Deletion of this subdomain in Ve1 did not result in loss of effector binding (Fig. 1b), indicating that the region between eLRR20-23 is not sufficient for the interaction with VdAve1. In addition to eLRR20-23, a second cluster of eLRRs (eLRR1-8) was shown to be required for Ve1 functionality ${ }^{19}$. Thus, it is conceivable that the physical interaction between Ve1 (and Ve2) and VdAve1 may be mediated by (a surface formed between) the two separate clusters of eLRRs. This is in contrast to the architecture of the ligand binding sites of other eLRRcontaining receptors. For example, crystallographic analyses demonstrated that the eLRRRK BRASSINOSTEROID INSENSITIVE 1 (BRI1) binds its brassinolide ligand via a hydrophibic groove formed by the island domain and the surrounding eLRRs20-25 $5^{16,47}$. Similarly, as 
elegantly shown by photoaffinity labeling, phytosulfokine (PSK) binding to the PSK receptor PSKR1 is mediated by the island domain ${ }^{48,49}$. Instead, the receptor kinases BAM1 and FLS2, which lack a non-LRR island domain, directly interact with the CLE9 and flg22 peptides, respectively, through a contiguous stretch of eLRRs ${ }^{50,51}$.

Due to the absence of cytoplasmic signaling domains, eLRR-RPs rely on the association with receptor kinases that function as adapters and co-receptors to activate appropriate responses upon ligand perception. Recent work has shown that SOBIR1 acts as a common adaptor to eLRR-RPs to form constitutive bimolecular receptor complexes ${ }^{20,21}$, including the immune receptors Cf-4, Cf- 9 and Eix 1 from tomato, RLP23 from Arabidopsis, as well as the Leptosphaeria maculans receptor 3 (LepR3) from oilseed rape 22,25,26,30. In addition, the Arabidopsis eLRR-RPs ReMAX, RLP30 and RLP42 require SOBIR1 for their function in immune signaling 27,28,52. Also Ve1 has been shown to associate with SOBIR1 in a ligandindependent manner ${ }^{18}$. Due to the high sequence conservation of the juxtamembrane C3 domain between various eLRR-RPs and because this region is important for Ve1 function, it has been hypothesized that the C3 domain is involved in the interaction with SOBIR $1^{19}$. However, this domain is not functional in Ve ${ }^{18}$. Nonetheless, like Ve1, Ve2 forms a ligand-independent complex with SOBIR1. This suggests that the amino acid residues (or their properties) required for SOBIR1 binding are conserved between Ve1 and the non-functional Ve2 receptor and that the association with SOBIR 1 may not be a determining factor of Ve1-mediated immunity ${ }^{18}$. Moreover, it does not explain the lack of Ve2 functionality in resistance to $V$. dahliae.

Upon ligand perception, eLRR-containing receptor molecules recruit additional receptor complex components to activate intracellular signaling cascades. BAK1 acts as a common co-receptor to both eLRR-RPs and eLRR-RKs to regulate developmental as well as immunological processes ${ }^{53}$. We show here that the Ve1-SOBIR1 complex recruits BAK 1 in a ligand-dependent manner (Fig. 3), as has previously been demonstrated for FLS2, Cf-4, and $\mathrm{RLP} 23^{24,25,54}$. This is in accordance with the previous finding that BAK1 silencing impairs Ve1mediated immunity in tomato ${ }^{6}$. Considering that ligand-dependent complex formation with BAK1 appears to be a common feature of eLRR receptor-mediated immunity, and since Ve2 interacts with the SOBIR1 receptor adapter, it is tempting to speculate that Ve2 does not activate immune signaling upon VdAve1 binding because it fails to recruit BAK1. This hypothesis is supported by the finding that the C-terminus of Ve2, which is 91 amino acids longer than that of Ve1, interferes with Ve1 function in domain swap mutants ${ }^{18}$. However, while Ve1 and Ve2 appear to have the same subcellular localization in tobacco cells, we cannot exclude the possibility that the PEST-like sequence in the Ve2 C-terminus, which is commonly found in proteins with short half-lives ${ }^{5}$, contributes to Ve2 degradation and, thus, renders it non-functional. Future studies, especially the structural analysis of the ligand-induced Ve1 receptor complex, will help to address this question. 


\section{Materials}

\section{Plant material}

Nicotiana benthamiana plants were grown in the greenhouse at $21^{\circ} \mathrm{C} / 19^{\circ} \mathrm{C}$ during $16 / 8$ hours day/night periods, respectively, with $70 \%$ relative humidity. When natural light intensities dropped below $150 \mathrm{~W} / \mathrm{m}^{2}$, supplemental light was provided at $100 \mathrm{~W} / \mathrm{m}^{2}$. For agroinfiltration, plants were transferred to climate chambers set to $21^{\circ} \mathrm{C} / 19^{\circ} \mathrm{C}$ during $12 / 12$ hours day/night periods, respectively, with $70 \%$ relative humidity.

\section{Constructs for Agrobacterium tumefaciens-mediated transient expression}

Receptor expression constructs pSol2095:Ve1-eGFP, pB7K40:Ve1-HA ${ }_{3^{\prime}}$ pB7K40:Ve2-HA ${ }_{3^{\prime}}$ pBIN-KS-35S:RLP30-HA ${ }_{3^{\prime}}$ pGWB20:SIBAK1-CMyc ${ }_{10^{\prime}}$ and pBIN-KS-35S:SISOBIR1-eGFP as well as effector expression constructs pSol2092:GFP-VdAve1, pSol2092:GFP-VnAve1, pSol2092:GFP-FoAve1, pSol2092:GFP-CbAve1, pSol2092:GFP-ChAve1, pSol2092:GFPXacPNP and pSol2092:SP-GFP were previously described 12,18,22,24,32. C-terminal VdAve1 deletion mutants were cloned from pEX-A2:SP-GFP-VdAve1 synthesized by Eurofins (Ebersberg, Germany) using the primers shown in Supplementary Table 1.The PCR-amplified fragments were cloned into pDONR207 by using the Gateway BP Clonase II Enzyme Mix (Invitrogen, California, USA) to generate the entry vectors pDONR207::VdAve1 $1^{\Delta 126-134}$ and pDONR207::VdAve1 ${ }^{\Delta 117-134}$. The constructs were verified by DNA sequencing (Eurofins Genomics, Ebersberg, Germany) and transferred into the Gateway-compatible destination vector pSol2092 ${ }^{12}$ by using Gateway ${ }^{\circ}$ LR Clonase II Enzyme Mix (Invitrogen, California, USA).

\section{A. tumefaciens-mediated transient expression (agroinfiltration)}

Overnight cultures of $A$. tumefaciens strain GV3101 carrying the respective expression constructs were centrifuged and pellets were re-suspended in MMA medium (2\% sucrose, $0.5 \%$ Murashige \& Skoog salts (Duchefa Biochemie, Haarlem, The Netherlands), $10 \mathrm{mM}$ MES, $200 \mu \mathrm{M}$ acetosyringone, $\mathrm{pH} 5.6$ with $\mathrm{NaOH}$ ). Bacterial suspensions were mixed at a 1:1 ratio for co-expression of effector with receptor genes. For co-expression of Ve1-HA/ Ve2-HA, SISOBIR1-GFP, and BAK1-CMyc, bacterial suspensions were mixed at a 1:1:1 ratio. Mixtures were infiltrated into leaves of 4 to 6 week-old $N$. benthamiana plants. After 2448 hours of co-expression, infiltrated leaves were harvested and snap-frozen in liquid $\mathrm{N}_{2}$.

\section{Co-immunopurifications and immunoblotting}

Ground leaf material was used for total protein extraction with $2 \mathrm{~mL}$ extraction buffer (150 mM Tris, $150 \mathrm{mM} \mathrm{NaCl}$, 10\% glycerol, 10 mM EDTA, 10 mM DTT, 0.5\% PVPP, protease inhibitor cocktail (Roche, Mannheim, Germany), 1\% IGEPAL CA-630 (NP40), pH 7.5) per gram 
of leaf material. Five $\mathrm{mL}$ total protein extracts were incubated with $20 \mu \mathrm{L}$ Pierce ${ }^{\mathrm{TM}}$ Anti-HA magnetic beads (Thermo Scientific, Eindhoven, The Netherlands) or GFP-Trap ${ }^{\circledR} \_$A beads (Chromotek, Planegg-Martinsried, Germany) shaking for 1 hour at $4^{\circ} \mathrm{C}$. Beads were washed three times with $1 \mathrm{xTBS}-\mathrm{T}(20)$. Proteins were eluted from beads in $30 \mu \mathrm{L} 2 \mathrm{x}$ Laemmli sample buffer (120 mM Tris-HCl pH 6.8, 4\% SDS, 10\% glycerol, 0.02\% (w/v) bromophenol blue) by boiling at $95^{\circ} \mathrm{C}$ for $5 \mathrm{~min}$. Proteins were separated on $4-20 \%$ gradient Mini-PROTEAN ${ }^{\circ} \mathrm{TGX}^{\circ}$ Stain-Free ${ }^{\mathrm{TM}}$ Precast Protein Gels (BioRad, Veenendaal, The Netherlands) in 1x Tris/glycine/ SDS running buffer (BioRad, Veenendaal, The Netherlands) at $150 \mathrm{~V}$ for 1 hour. Protein bands were transferred onto Immun-Blot PVDF membranes (BioRad, Veenendaal, The Netherlands) in blotting buffer (247.7 mM Tris, $1.92 \mathrm{M}$ glycine, 10\% ethanol) using the Trans-Blot ${ }^{\circ}$ Turbo $^{\text {TM }}$ Blotting System (BioRad, Veenendaal, The Netherlands) for 30 min at 25 V. Membranes were blocked in 4\% bovine serum albumin (BSA) in 1x TBS-T(20). To detect GFP-tagged proteins, blots were incubated with 1:5,000 diluted aGFP-HRP (Anti-GFP-HRP, 130-091-833, MACS antibodies). HA fusion proteins were detected with 1:2,500 diluted aHA-HRP (3F10; Roche, Mannheim, Germany) and cMyc-tagged proteins were detected with 1:5,000 a-cMyc-HRP (Thermo Scientific, Eindhoven, The Netherlands). Bands were visualized using SuperSignal West Femto Chemiluminescent Substrate (Thermo Scientific, Eindhoven, The Netherlands).

\section{Recombinant protein}

GST-tagged Ave1 was heterologously produced in insect cells and purified by GenScript (Piscataway, NJ, USA). GST was purchased from Genscript (Piscataway, NJ, USA).

\section{Acknowledgements}

The authors thank Bert Essenstam (Unifarm) for excellent plant care. Work in the laboratory of B.P.H.J. Thomma is supported by the Research Council for Earth and Life sciences (ALW) of the Netherlands Organization for Scientific Research (NWO). 


\section{References}

1 Macho, A. P. \& Zipfel, C. Plant PRRs and the activation of innate immune signaling. Mol. Cell 54, 263-272, doi:10.1016/j.molcel.2014.03.028 (2014).

2 Cook, D. E., Mesarich, C. H. \& Thomma, B P. H. J. Understanding plant immunity as a surveillance system to detect invasion. Annu. Rev. Phytopathol. 53, 541-563, doi:10.1146/annurevphyto-080614-120114 (2015).

3 Dou, D. \& Zhou, J. M. Phytopathogen effectors subverting host immunity: different foes, similar battleground. Cell Host Microbe 12, 484-495, doi:10.1016/j.chom.2012.09.003 (2012).

4 Rovenich, H., Boshoven, J. C. \& Thomma, B. P. H. J. Filamentous pathogen effector functions: of pathogens, hosts and microbiomes. Curr. Opin. Plant Biol. 20, 96-103, doi:10.1016/j. pbi.2014.05.001 (2014).

5 Kawchuk, L. M. et al. Tomato Ve disease resistance genes encode cell surface-like receptors. Proc. Natl. Acad. Sci. USA 98, 6511-6515, doi:10.1073/ pnas.091114198 (2001).

6 Fradin, E. F. et al. Genetic dissection of Verticillium wilt resistance mediated by tomato Ve1. Plant Physiol. 150, 320-332, doi:10.1104/pp.109.136762 (2009).

7 Monaghan, J. \& Zipfel, C. Plant pattern recognition receptor complexes at the plasma membrane. Curr. Opin. Plant Biol. 15, 349-357, doi:10.1016/j. pbi.2012.05.006 (2012).

8 Ellendorff, U., Fradin, E. F., de Jonge, R. \& Thomma, B. P. H. J. RNA silencing is required for Arabidopsis defence against Verticillium wilt disease. J. Exp. Bot. 60, 591-602, doi:10.1093/jxb/ern306 (2009).

9 Fradin, E. F. et al. Interfamily transfer of tomato Ve1 mediates Verticillium resistance in Arabidopsis. Plant Physiol 156, 2255-2265, doi:10.1104/ pp.111.180067 (2011).

10 Song, Y. et al. Broad taxonomic characterization of Verticillium wilt resistance genes reveals an ancient origin of the tomato Ve1 immune receptor. Mol. Plant Pathol. 18, 195-209, doi:10.1111/mpp.12390 (2017).

11 de Jonge, R. et al. Tomato immune receptor Ve1 recognizes effector of multiple fungal pathogens uncovered by genome and RNA sequencing. Proc. Natl. Acad. Sci. USA 109, 5110-5115, doi:10.1073/ pnas.1119623109 (2012).
12 Zhang, Z. et al. Optimized agroinfiltration and virus-induced gene silencing to study Ve1mediated Verticillium resistance in tobacco. Mol. Plant-Microbe Interact. 26, 182-190, doi:10.1094/ MPMI-06-12-0161-R (2013)

13 Zhang, Z. et al. Ve1-mediated resistance against Verticillium does not involve a hypersensitive response in Arabidopsis. Mol. Plant Pathol. 14, 719-727, doi:10.1111/mpp.12042 (2013).

14 Kobe, B. \& Kajava, A. V. The leucine-rich repeat as a protein recognition motif. Curr. Opin. Struct. Biol. 11, 725-732 (2001).

15 Dunning, F. M., Sun, W., Jansen, K. L., Helft, L. \& Bent, A. F. Identification and mutational analysis of Arabidopsis FLS2 leucine-rich repeat domain residues that contribute to flagellin perception. Plant Cell 19, 3297-3313, doi:10.1105/ tpc.106.048801 (2007).

16 Hothorn, M. et al. Structural basis of steroid hormone perception by the receptor kinase BRI1 Nature 474, 467-471, doi:10.1038/nature10153 (2011).

17 Wulff, B. B. et al. The major specificity-determining amino acids of the tomato Cf- 9 disease resistance protein are at hypervariable solvent-exposed positions in the central leucine-rich repeats. Mol. Plant-Microbe Interact. 22, 1203-1213, doi:10.1094/MPMI-22-10-1203 (2009).

18 Fradin, E. F. et al. Functional analysis of the tomato immune receptor Ve1 through domain swaps with its non-functional homolog Ve2. PLoS ONE 9, e88208, doi:10.1371/journal.pone.0088208 (2014).

19 Zhang, Z., Song, Y., Liu, C. M. \& Thomma, B. P. H. J. Mutational analysis of the Ve1 immune receptor that mediates Verticillium resistance in tomato. PLoS ONE 9, e99511, doi:10.1371/journal. pone.0099511 (2014).

20 Gust, A. A. \& Felix, G. Receptor like proteins associate with SOBIR1-type of adaptors to form bimolecular receptor kinases. Curr. Opin. Plant Biol. 21, 104-111, doi:10.1016/j.pbi.2014.07.007 (2014) 21 Liebrand, T. W., van den Burg, H. A. \& Joosten, M. H. A. J. Two for all: receptor-associated kinases SOBIR1 and BAK1. Trends Plant Sci. 19, 123-132, doi:10.1016/j.tplants.2013.10.003 (2014). 
22 Liebrand, T. W. et al. Receptor-like kinase SOBIR1/ EVR interacts with receptor-like proteins in plant immunity against fungal infection. Proc. Natl. Acad. Sci. USA 110, 10010-10015, doi:10.1073/ pnas.1220015110 (2013).

23 Couto, D. \& Zipfel, C. Regulation of pattern recognition receptor signalling in plants. Nat. Rev. Immunol. 16, 537-552, doi:10.1038/nri.2016.77 (2016).

24 Postma, J. et al. Avr4 promotes Cf-4 receptor-like protein association with the BAK1/SERK3 receptorlike kinase to initiate receptor endocytosis and plant immunity. New Phytol. 210, 627-642, doi:10.1111/nph.13802 (2016).

25 Albert, I. et al. An RLP23-SOBIR1-BAK1 complex mediates NLP-triggered immunity. Nat. Plants $\mathbf{1}$ 15140, doi:10.1038/nplants.2015.140 (2015).

$26 \mathrm{Bi}, \mathrm{G}$. et al. Arabidopsis thaliana receptor-like protein AtRLP23 associates with the receptor-like kinase AtSOBIR1. Plant Signal. Behav. 9, e27937 (2014).

27 Zhang, W. et al. Arabidopsis receptor-like protein30 and receptor-like kinase suppressor of BIR1-1/EVERSHED mediate innate immunity to necrotrophic fungi. Plant Cell 25, 4227-4241, doi:10.1105/tpc.113.117010 (2013).

28 Jehle, A. K. et al. The receptor-like protein ReMAX of Arabidopsis detects the microbe-associated molecular pattern eMax from Xanthomonas. Plant Cell 25, 2330-2340, doi:10.1105/tpc.113.110833 (2013).

29 Peng, K. C., Wang, C. W., Wu, C. H., Huang, C. T. \& Liou, R. F. Tomato SOBIR1/EVR homologs are involved in elicitin perception and plant defense against the oomycete pathogen Phytophthora parasitica. Mol. Plant-Microbe Interact. 28, 913-926, doi:10.1094/ MPMI-12-14-0405-R (2015).

$30 \mathrm{Ma}, \mathrm{L}$. \& Borhan, M. H. The receptor-like kinase SOBIR1 interacts with Brassica napus LepR3 and is required for Leptosphaeria maculans AvrLm1triggered immunity. Front Plant Sci. 6, 933, doi:10.3389/fpls.2015.00933 (2015).

31 Bar, M., Sharfman, M., Ron, M. \& Avni, A. BAK1 is required for the attenuation of ethylene-inducing xylanase (Eix)-induced defense responses by the decoy receptor LeEix1. Plant J. 63, 791-800, doi:10.1111/j.1365-313X.2010.04282.x (2010).

32 Song, $Y$. et al. Tomato immune receptor Ve1 recognizes surface-exposed co-localized $\mathrm{N}$ - and C-termini of Verticillium dahliae effector Ave1. bioRxiv, doi:https://doi.org/10.1101/103473 (2017).
33 de Jonge, R. et al. Conserved fungal LysM effector Ecp6 prevents chitin-triggered immunity in plants. Science 329, 953-955, doi:10.1126/ science.1190859 (2010).

34 Van der Hoorn, R. A., Roth, R. \& De Wit, P. J. G. M. Identification of distinct specificity determinants in resistance protein $\mathrm{Cf}-4$ allows construction of a Cf-9 mutant that confers recognition of avirulence protein Avr4. Plant Cell 13, 273-285 (2001).

35 Albert, M. et al. Arabidopsis thaliana pattern recognition receptors for bacterial elongation factor Tu and flagellin can be combined to form functional chimeric receptors. J. Biol. Chem. 285, 19035-19042, doi:10.1074/jbc.M110.124800 (2010).

36 Ron, M. \& Avni, A. The receptor for the fungal elicitor ethylene-inducing xylanase is a member of a resistance-like gene family in tomato. Plant Cell 16, 1604-1615, doi:10.1105/tpc.022475 (2004).

37 Chinchilla, D., Bauer, Z., Regenass, M., Boller, T. \& Felix, G. The Arabidopsis receptor kinase FLS2 binds flg22 and determines the specificity of flagellin perception. Plant Cell 18, 465-476, doi:10.1105/tpc.105.036574 (2006).

38 Rooney, H. C. et al. Cladosporium Avr2 inhibits tomato Rcr3 protease required for Cf-2dependent disease resistance. Science 308, 17831786, doi:10.1126/science.1111404 (2005).

39 Shabab, M. et al. Fungal effector protein AVR2 targets diversifying defense-related cys proteases of tomato. Plant Cell 20, 1169-1183, doi:10.1105/ tpc.107.056325 (2008).

40 van Esse, H. P. et al. The Cladosporium fulvum virulence protein Avr2 inhibits host proteases required for basal defense. Plant Cell 20, 19481963, doi:10.1105/tpc.108.059394 (2008).

41 Luderer, R. et al. No evidence for binding between resistance gene product $\mathrm{Cf}-9$ of tomato and avirulence gene product AVR9 of Cladosporium fulvum. Mol. Plant-Microbe Interact. 14, 867-876, doi:10.1094/MPMI.2001.14.7.867 (2001).

42 Kooman-Gersmann, M. et al. Correlation between binding affinity and necrosis-inducing activity of mutant AVR9 peptide elicitors. Plant Physiol. 117, 609-618 (1998).

43 Dodds, P. N. \& Rathjen, J. P. Plant immunity: towards an integrated view of plant-pathogen interactions. Nat. Rev. Genet. 11, 539-548, doi:10.1038/nrg2812 (2010). 
44 Axtell, M. J. \& Staskawicz, B. J. Initiation of RPS2specified disease resistance in Arabidopsis is coupled to the AvrRpt2-directed elimination of RIN4. Cell 112, 369-377 (2003).

45 Mackey, D., Belkhadir, Y., Alonso, J. M., Ecker, J. R. \& Dangl, J. L. Arabidopsis RIN4 is a target of the type III virulence effector AvrRpt2 and modulates RPS2-mediated resistance. Cell 112, 379-389 (2003).

46 Mackey, D., Holt, B. F., 3rd, Wiig, A. \& Dangl, J. L. RIN4 interacts with Pseudomonas syringae type III effector molecules and is required for RPM1mediated resistance in Arabidopsis. Cell 108, 743754 (2002).

47 She, J. et al. Structural insight into brassinosteroid perception by BRI1. Nature 474, 472-476, doi:10.1038/nature10178 (2011).

48 Matsubayashi, Y., Ogawa, M., Morita, A. \& Sakagami, Y. An LRR receptor kinase involved in perception of a peptide plant hormone, phytosulfokine. Science 296, 1470-1472, doi:10.1126/science.1069607 (2002).

49 Shinohara, H., Ogawa, M., Sakagami, Y. \& Matsubayashi, Y. Identification of ligand binding site of phytosulfokine receptor by on-column photoaffinity labeling. J. Biol. Chem. 282, 124-131, doi:10.1074/jbc.M604558200 (2007).
50 Shinohara, H., Moriyama, Y., Ohyama, K. \& Matsubayashi, Y. Biochemical mapping of a ligand-binding domain within Arabidopsis BAM1 reveals diversified ligand recognition mechanisms of plant LRR-RKs. Plant J. 70, 845-854, doi:10.1111/ j.1365-313X.2012.04934.x (2012).

51 Sun, Y. et al. Structural basis for flg22-induced activation of the Arabidopsis FLS2-BAK1 immune complex. Science 342, 624-628, doi:10.1126/ science.1243825 (2013).

52 Zhang, L. et al. Fungal endopolygalacturonases are recognized as microbe-associated molecular patterns by the Arabidopsis receptor-like protein RESPONSIVENESS TO BOTRYTIS POLYGALACTURONASES1. Plant Physiol. 164, 352364, doi:10.1104/pp.113.230698 (2014).

$53 \mathrm{Ma}, \mathrm{X} ., \mathrm{Xu}, \mathrm{G} ., \mathrm{He}, \mathrm{P}$. \& Shan, L. SERKing Coreceptors for Receptors. Trends Plant Sci. 21, 1017-1033, doi:10.1016/j.tplants.2016.08.014 (2016).

54 Chinchilla, D. et al. A flagellin-induced complex of the receptor FLS2 and BAK1 initiates plant defence. Nature 448, 497-500, doi:10.1038/ nature05999 (2007). 


\section{Supplementary data}

a

\begin{tabular}{|l|l|l|}
\hline SP & GFP & VdAve1 \\
\hline
\end{tabular}

b
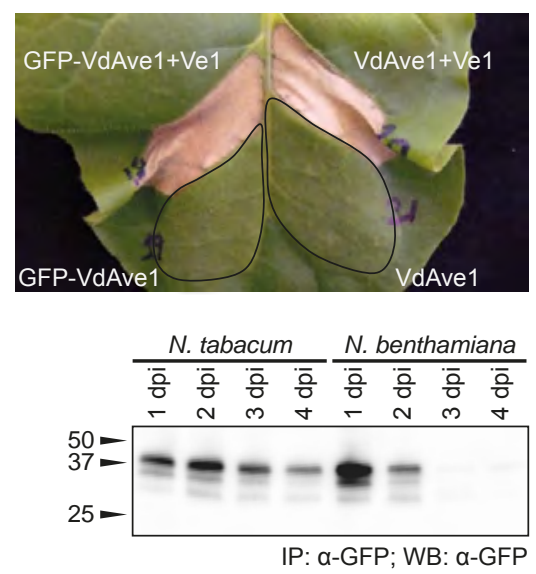

SUPPLEMENTARY FIGURE 1 | GFP-tagged VdAve1 is expressed and recognized by Ve1. a, Graphic representation of the GFP-VdAve1 construct used for A. tumefaciens-mediated transient expression in $N$. tabacum and $N$. benthamiana. b, Hypersensitive response (HR) following co-expression of GFP-tagged or untagged VdAve1 with Ve1 in N. tabacum at 6 dpi. Expression of GFP-VdAve1 or Ave1 alone did not result in HR. c, Western blot (WB) showing GFP-VdAve1 stability at 1, 2, 3, and 4 days following $A$. tumefaciensmediated transient expression in N. tabacum and $N$. benthamiana. GFP-VdAve1 was immunopurified with a-GFP Trap beads from total protein extracts. 



\title{
Chapter 7
}

\author{
General discussion
}


This thesis provides a detailed insight into the mechanisms underlying chitin-triggered immunity in tomato, and the strategies that evolved in the two fungal pathogens Cladosporium fulvum and Verticillium dahliae to avoid immune activation. The presented work represents a significant advancement in our understanding of how fungal microbes manipulate their host's immune system by actively targeting its constituents, including chitinases and chitin receptors. In the following sections, these findings are placed into the broader context of immune recognition of cell wall-derived molecules and the evolution of fungi as well as oomycetes, collectively referred to as filamentous microbes, toward its suppression.

\section{Introduction}

The first intimate contact between host plants and filamentous microbes is often established in the apoplast; the extracellular spaces of plant tissues. While the apoplast provides nutrients and shelter to microbial inhabitants, it also represents a hostile environment with host-derived toxins and hydrolytic enzymes such as chitinases and glucanases that affect pathogen cell wall integrity (Fig. 1a). Moreover, the released cell wall fragments may serve as invasion patterns (IPs) and activate host immune receptors ${ }^{1-3}$. Consequently, microbial mechanisms evolved to circumvent cell wall-triggered immune responses and support host colonization.

\section{Recognition of glycan oligomers is mediated by closely related receptors}

Chitin is an important structural building block of fungal cell walls and a well-known elicitor of immune responses in plants' ${ }^{1}$. Chitin perception by surface-localized lysin motif (LysM)-containing receptors and subsequent immune activation are well understood ${ }^{1,4}$. In rice, chitin perception is mediated by the LysM receptor OsCEBiP, which lacks an intracellular signaling domain ${ }^{5}$.Two OsCEBiP molecules simultaneously bind a single chitin heptamer-octamer, resulting in the recruitment of the LysM receptor kinase OsCERK1 (Fig. $1 \mathrm{~b})^{6}$. OsCERK 1 is then phosphorylated and initiates immune signaling ${ }^{6,7}$. Additionally, OsCERK1 forms multimeric complexes with LysM receptors implicated in peptidoglycan perception ${ }^{8,9}$. Thus, OsCERK1 functions as signal transduction adaptor to multiple LysM immune receptors in response to the recognition of various microbial glycans.

Despite considerable efforts, the proposed composition of the chitin receptor complex in Arabidopsis remains controversial. Similar to rice, the Arabidopsis CERK1 homolog plays a central role in chitin-triggered immune signaling ${ }^{10}$. However, unlike OsCERK1, AtCERK1 was reported to directly bind long chain chitin oligomers, albeit with relatively 
low affinity ${ }^{11-13}$. Recently, the LysM receptor AtLYK5, which lacks an active kinase domain, was proposed as primary chitin receptor instead of AtCERK1 based on its higher affinity for chitin ${ }^{14}$. Similar to rice, AtLYK5 forms a chitin-induced heteromeric complex with AtCERK1, triggering AtCERK1 phosphorylation and immune signaling activation. Since AtCERK1 functions in additional processes including peptidoglycan recognition and bacterial immunity ${ }^{15}$, AtCERK1 may be a receptor adaptor, equivalent to OsCERK1. As in Arabidopsis, chitin recognition in tomato is mediated by a LysM receptor, called SILYK4, which lacks an active intracellular kinase domain (Chapter 4). Thus, it is likely that SILYK4 recruits a second receptor component, such as CERK1 in Arabidopsis and rice, to initiate immune signaling upon chitin perception.

In contrast to chitin, little is known about glucan recognition in plants. Due to their abundance in fungal and oomycete cell walls, glucan oligosaccharides likely evoke plant immune responses (Fig. 1b) ${ }^{2}$. The only known ß-glucan receptor, soybean GBP, does not contain LysM domains but specifically binds a hepta-ß-glucoside via a glucan-binding site $^{16}$. As GBP lacks an intracellular signaling domain, it is probably part of a multimeric receptor complex at the cell surface, analogous to chitin perception complexes.

Interestingly, in addition to immunity, LysM receptors enhance the efficiency of the establishment of mutualistic symbioses triggered by short chain chitin fragments and chitin-related lipochitooligosaccharides, called Myc factors ${ }^{17,18}$. Some LysM receptors play a dual role in the perception of Myc factors and closely-related bacterial Nod factors that trigger root nodule symbiosis ${ }^{17}$. Moreover, OsCERK1 has recently been shown to function both in immunity and mutualism ${ }^{19,20}$.

Biochemical analyses showed that several LysM receptors are glycosylated with highmannose-type glycans or complex-type $\mathrm{N}$-glycans ${ }^{5,21}$. However, to date no functional role has been ascribed to LysM receptor $N$-glycosylation ${ }^{22}$. Thus, the importance of such posttranscriptional modifications for ligand binding and receptor function still needs to be determined.

Collectively, the findings discussed above highlight the central role of LysM receptors in the recognition of chitin-derived microbial ligands. Future work will have to determine whether the perception of other cell wall glycans is similarly mediated by LysM receptors or by different classes of receptor proteins.

\section{Microbial strategies to evade glycan-triggered immunity}

The recognition of microbe-derived glycan molecules activates immune responses that hamper host colonization. In order to overcome or bypass such responses, filamentous microbes employ different strategies, comprising cell wall modifications and the secretion 
of glycan-binding effector proteins. These effectors either sequester glycan elicitors and prevent their recognition or shield the cell wall from hydrolysis. Additionally, filamentous microbes secrete effectors that inhibit hydrolytic enzyme activity and proteases that cleave hydrolytic enzymes.

\section{Cell wall modifications}

The cell wall of filamentous microbes is vital for their growth, morphogenesis and survival. Despite variations between species, chitin and B-glucan are the most abundant cell wall components. Their recognition by host surface-localized receptors activates detrimental immune responses. In contrast, deacetylated chitin (chitosan) and a-glucans are much weaker inducers of defense responses in most plants ${ }^{2}$. Moreover, chitosan is a poor substrate for plant chitinase ${ }^{23}$. Therefore, conversion of chitin to chitosan may protect hyphae against cell wall hydrolysis, and limit the release of plant immune signaling elicitors (Fig. 1d)'. Affinity labeling of invasive hyphae of particular fungal pathogens with a chitosan-specific antibody but not a chitin-binding probe suggested that cell wall chitin was replaced by chitosan ${ }^{24}$. Moreover, genome analyses of the ectomycorrhizal fungus Laccaria bicolor and the phytopathogen Melampsora larici-populina revealed an expanded repertoire of chitin deacetylases ${ }^{25,26}$. Thus, chitin deacetylation may represent a widespread mechanism of fungal cell wall protection and plant immune signaling evasion (Fig. 1d).

Rather than deacetylating chitin, some pathogens accumulate $a-1,3$-glucan on the surface of infectious hyphae (Fig. 1c) ${ }^{27}$. Since mutants with reduced levels of $a-1,3$-glucan are more susceptible to chitinases and display reduced virulence, $a-1,3$-glucan may protect fungal cell walls by masking chitin and B-glucans (Fig. 1C) ${ }^{27}$. An additional strategy for evading B-glucan-triggered immunity is the depletion of this polymer at the cell wall of biotrophic hyphae. While $\beta-1,3$-glucan synthesis is required for cell wall rigidity in appressoria and fast-growing necrotrophic hyphae of the maize pathogen Colletotrichum graminicola, during biotrophic development its synthesis is rigorously downregulated ${ }^{28}$.

In conclusion, by converting, depleting or disguising highly immunoactive cell wall components, filamentous microbes can prevent cell wall hydrolysis and the release of host immune signaling elicitors. 

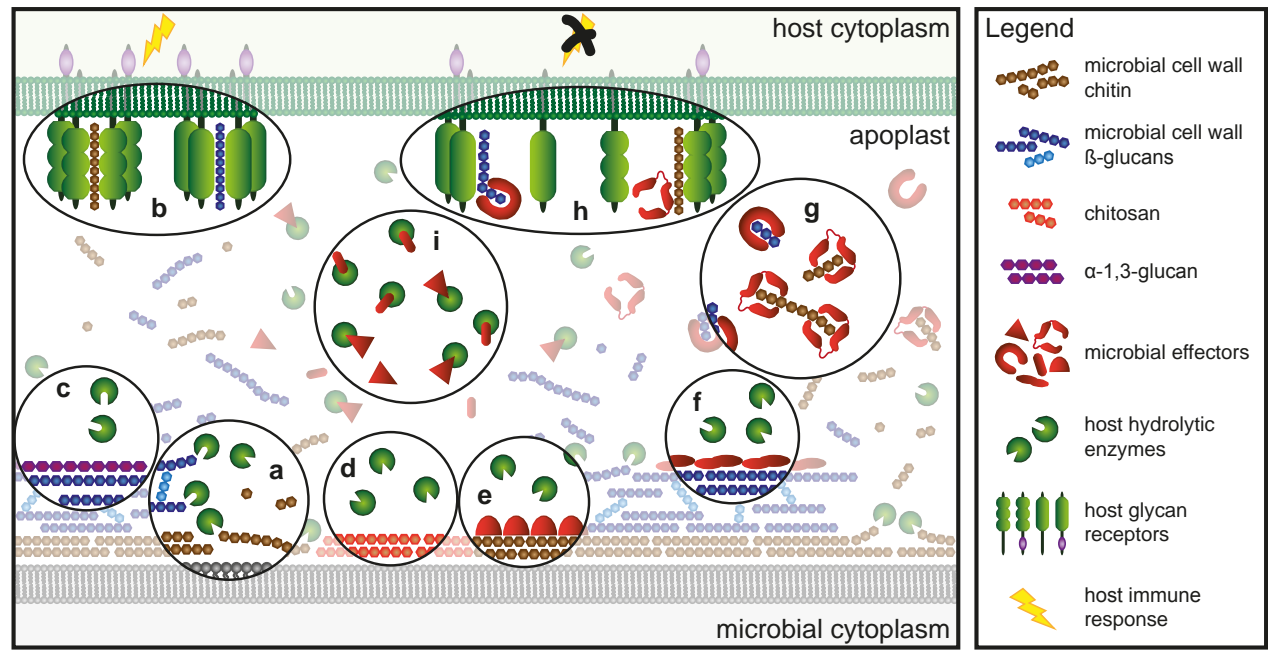

FIGURE 1 | Illustration of potential mechanisms underlying the activation of glycan-triggered host immune responses and microbial strategies to evade glycan recognition. (a) Microbial cell wall glycans are targeted by host-derived hydrolytic enzymes, including glucanases and chitinases, resulting in the release of glycan fragments. (b) Recognition of chitin oligomers by plasma membrane-localized host receptors results in the assembly of oligomeric receptor complexes, leading to the activation of immune responses. Similarly, recognition of ß-glucan fragments is anticipated to occur at the host cell surface. (c) Microbial cell wall remodeling may reduce the access of hydrolytic enzymes to particular cell wall glycans to prevent their hydrolysis, such as accumulation of a-1,3-glucan at the surface. (d) Certain fungal species convert chitin to chitosan, which is less immunogenic and a poor substrate to chitinases. Microbial secretion of glycan-binding effector proteins may shield cell wall chitin (e) or glucans (f) from hydrolysis, or sequester released glycan fragments to prevent their recognition (g). (h) Particular effectors may prevent the assembly or activation of host receptor complexes by inhibiting ligand-induced receptor dimerization. (i) Finally, microbes may secrete effectors that directly inhibit host hydrolytic enzymes.

\section{Sequestration of cell wall-derived glycan fragments}

Chitin-binding LysM effector proteins are versatile suppressors of chitin-triggered immunity (Fig. 1e,g,h). The Cladosporium fulvum LysM effector Ecp6 is one of the most abundant apoplastic proteins during tomato infection that binds chitin with high specificity and perturbs chitin-triggered immune responses ${ }^{29,30}$. Crystallization of Ecp6 demonstrated that two of its three LysM domains form a composite binding site with ultrahigh (pM) chitin affinity, enabling Ecp6 to outcompete host immune receptors for chitin binding ${ }^{31}$. Interestingly, the singular LysM2 domain of Ecp6, which is not involved in intramolecular chitin binding, retains the capacity to suppress chitin-triggered immune responses. Thus, it has been hypothesized that LysM2 of Ecp6 may interfere with chitininduced host receptor dimerization to prevent activation of immune signaling (Fig. $1 \mathrm{~h})^{31,32}$. First evidence in support of this hypothesis results from functional analyses in Arabidopsis that suggest that Ecp6 does not prevent AtCERK1 phosphorylation but 
affects the internalization of AtLYK5 in a ligand-dependent manner (Chapter 3). With the identification of the tomato chitin receptor, the biological relevance of these findings can now be confirmed in the $C$. fulvum host.

Like Ecp6, Magnaporthe oryzae Slp1, Zymoseptoria tritici Mg3LysM, and Colletotrichum higginsianum ChELP1 and ChELP2 deregulate chitin-triggered immunity ${ }^{33-35}$. Therefore, the ability to interfere with chitin recognition and immunity is conserved between fungal pathogens of different genera. Interestingly, SIp1 stability and activity depend on $N$-glycosylation by the a-1,3-mannosyltransferase AGL3 ${ }^{36}$. Similarly, ChELP1 and ChELP2 appear to be $\mathrm{N}$-glycosylated ${ }^{35}$ suggesting that the addition of glycans may be an important feature of LysM effector function.

Importantly, the root endophyte Serendipita indica has recently been shown to secrete a fungal-specific lectin, FGB1, to compete with Arabidopsis and barley $\beta$-glucan receptors to suppress $\beta$-glucan-induced defenses in root and leaf tissues ${ }^{37}$. This suggests that, in addition to chitin, recognition of ß-glucans sparked the evolution of ß-glucan-binding effectors to interfere with ß-glucan-triggered immune responses (Fig. 1g,h).

\section{Cell wall masking and inhibition of hydrolytic enzyme activity}

In addition to chitooligosaccharide sequestration, Z. tritici Mg3LysM protects fungal hyphae from degradation by plant chitinases ${ }^{33}$. Z. tritici secretes a second LysM effector comprising a single LysM domain, called Mg1 LysM, which displays protective activity as well. Although the molecular mechanism underlying hyphal protection is unknown, these LysM effectors may form chitin-dependent oligomeric chains as protective layers around invasive hyphae (Fig. 1e) ${ }^{1}$. This was previously demonstrated for the C. fulvum effector Avr4 and a Pseudocercospora fijiensis orthologue that bind polymeric chitin via an invertebrate chitin-binding site to protect hyphae against hydrolysis ${ }^{38,39}$. Similarly, effectors to mask cell wall ß-glucan may exist (Fig. 1f).

Alternatively, Mg3LysM and Mg1LysM may protect hyphae through direct inhibition of chitinase activity (Fig. 1i). In silico analyses of structural models demonstrated that particular active site residues of plant class I chitinases are subject to positive selection pressure, likely imposed by microbial chitinase inhibitors ${ }^{40}$. Intriguingly, the effector VdAve 1 of the vascular pathogen $V$. dahliae, which belongs to a group of ubiquitous plant natriuretic peptide (PNP)-containing proteins, is able to inhibit a class I chitinase of tomato (Chapter 5). This function is not displayed by its closest homolog from the saprotroph $V$. nubilum, and explains the earlier observation that core LysM effectors of $V$. dahliae are not expressed in planta, and thus do not contribute to virulence through suppression of chitin-triggered immunity ${ }^{41}$. Additionally, fungalysin metalloproteases can cleave class IV chitinases ${ }^{42}$. In Fusarium oxysporium f.sp. lycopersici a serine protease and metalloprotease contribute to virulence and proteolytically cleave extracellular tomato chitinases ${ }^{43}$. Similar 
activities occur in other tomato pathogens, suggesting that targeting of chitinases is a general strategy to protect cell walls ${ }^{43}$.

Further evidence for the direct inhibition of plant hydrolytic enzymes is provided by the glucanase inhibitor protein 1 (GIP1) of Phytophthora sojae, which directly inhibits a soybean endo-ß-1,3-glucanase (EGaseA) ${ }^{44}$. Similar to class I chitinases, Glycine EGaseA enzymes are under positive selection pressure and display high variability at residues in close proximity to the GIP1 inhibitor binding site ${ }^{45}$. Moreover, the cell death-inducing mycotoxin Fumonisin B1 of Fusarium verticillioides also inhibits basic ß-1,3-glucanases in maize embryos ${ }^{46}$. Thus, filamentous microbes evolved various protective mechanisms against host hydrolytic enzymes, including chitinases and glucanases (Fig. 1i).

\section{Evasion strategies of animal-infecting fungi}

In mammals, fungal cell walls are the main source of elicitors whose recognition leads to the activation of immune responses including phagocytosis and fungal clearance ${ }^{47}$. Recognition of chitin, ß-glucans and mannosylated proteins is mediated by various receptor molecules, including $\mathrm{C}$-type lectin and Toll-like receptors ${ }^{48}$. Consequently, also animal-infecting fungi evolved to mask and subvert host recognition. For example, Histoplasma capsulatum and Aspergillus fumigatus disguise immunoactive cell wall ß-glucans by depositing surface a-glucans that have little to no immunostimulatory activity ${ }^{49,50}$. In contrast, $A$. fumigatus conidia are coated with hyprophobins that are covalently bound to cell wall polysaccharides to prevent immune recognition ${ }^{51}$.

The lack of an identified chitosan receptor and conflicting reports on its immunological activity suggest that chitosan is not a major elicitor of immunity in animals ${ }^{52}$. Thus, chitin deacetylation to chitosan may shield invasive hyphae from recognition and hydrolytic cleavage. Supporting evidence is provided by a recent genomic survey on the opportunistic mammalian pathogen Rhizopus oryzae, which unveiled an expanded repertoire of chitin deacetylases ${ }^{53}$. Thus, in addition to cell wall masking, cell wall modifications may play an important role in the evasion of glycan-triggered immunity by animal pathogens.

Despite their ubiquitous occurrence, animal pathogen LysM effectors have thus far not been found to suppress host immunity. It has been hypothesized that infection of mammalian hosts does not rely on effector activity due to their low degree of host adaptation and absence of host specificity ${ }^{54}$. This is supported by the absence of LysM effector genes in various mammalian commensals ${ }^{55}$. 


\section{Conclusions}

The involvement of glucans, chitin, chitooligosaccharides and their derivatives in plant-microbe communication exemplifies how cell-surface exposed and free glycans regulate complex processes during disease manifestation or establishment of mutualistic symbiosis in plants. As many glycans have the potential to become immunogenic in host organisms, filamentous microbes rely on diverse strategies to prevent the activation of host immune responses. Both animal and plant-infecting filamentous microbes modify and mask immunoactive components of their cell walls. Moreover, the role of fungal pathogen-derived LysM effectors in the suppression of plant chitin-triggered immunity has been intensely studied. Recent evidence suggests that, depending on their cell wall composition, fungal and oomycete symbionts may employ a suite of effector proteins to protect, sequester, and mask chitin and glucan cell wall components alike. Moreover, some effectors inhibit hydrolytic enzyme activity. Whether this inhibition is based on effector binding to the enzyme-glycan complex or the active site itself remains to be determined. Therefore, the basic requirement to circumvent glycan-triggered immunity is a driving force in the convergent evolution of all filamentous microbes toward its suppression.

The use of oligosaccharides as signaling molecules in plants and the adoption of cellsurface glycan receptors illustrate how glycans have been adapted to serve new functions during evolution. Thus, better understanding of the factors that contribute to maintenance of harmful or beneficial microbial glycan structures is required as a basis for fundamental breakthroughs in knowledge of disease development and growth promotion in plants. The availability of a large number of filamentous microbe and plant genomes to identify putative microbial glycan-binding proteins and respective plant receptors, as well as recent advances in glycan research in animal systems, can now help study the role played by cellsurface sugars and their binding proteins in plant-filamentous microbe interactions.

\section{References}

1 Sánchez-Vallet, A., Mesters, J. R. \& Thomma, B. P. H. J. The battle for chitin recognition in plant-microbe interactions. FEMS Microbiol. Rev. 39, 171-183, doi:10.1093/femsre/fuu003 (2015).

2 Fesel, P. H. \& Zuccaro, A. ß-glucan: Crucial component of the fungal cell wall and elusive MAMP in plants. Fungal Genet. Biol., doi:10.1016/j. fgb.2015.12.004 (2015).

3 Cook, D. E., Mesarich, C. H. \& Thomma, B. 6 P. H. J. Understanding plant immunity as a surveillance system to detect invasion. Annu. Rev. Phytopathol. 53, 541-563, doi:10.1146/annurevphyto-080614-120114 (2015).
Antolín-Llovera, M. et al. Knowing your friends and foes - plant receptor-like kinases as initiators of symbiosis or defence. New Phytol. 204, 791-802, doi:10.1111/nph.13117 (2014).

5 Kaku, H. et al. Plant cells recognize chitin fragments for defense signaling through a plasma membrane receptor. Proc. Natl. Acad. Sci. USA 103, 1108611091, doi:10.1073/pnas.0508882103 (2006).

Hayafune, M. et al. Chitin-induced activation of immune signaling by the rice receptor CEBiP relies on a unique sandwich-type dimerization. Proc. Natl. Acad. Sci. USA 111, E404-413, doi:10.1073/ pnas.1312099111 (2014). 
7 Shimizu, T. et al. Two LysM receptor molecules, CEBiP and OsCERK1, cooperatively regulate chitin elicitor signaling in rice. Plant J. 64, 204-214, doi:10.1111/j.1365-313X.2010.04324.x (2010).

8 Ao, Y. et al. OsCERK1 and OsRLCK176 play important roles in peptidoglycan and chitin signaling in rice innate immunity. Plant J. 80, 1072-1084, doi:10.1111/tpj.12710 (2014).

9 Kouzai, Y. et al. Targeted gene disruption of OsCERK1 reveals its indispensable role in chitin perception and involvement in the peptidoglycan response and immunity in rice. Mol. Plant-Microbe Interact. 27, 975-982, doi:10.1094/Mpmi-03-140068-R (2014).

10 Miya, A. et al. CERK1, a LysM receptor kinase, is essential for chitin elicitor signaling in Arabidopsis. Proc. Natl. Acad. Sci. USA 104, 19613 19618, doi:10.1073/pnas.0705147104 (2007).

11 lizasa, E., Mitsutomi, M. \& Nagano, Y. Direct binding of a plant LysM receptor-like kinase, LysM RLK1/CERK1, to chitin in vitro. J. Biol. Chem. 285 , doi:10.1074/Jbc.M109.027540 (2010).

12 Petutschnig, E. K., Jones, A. M., Serazetdinova, L., Lipka, U. \& Lipka, V. The lysin motif receptor-like kinase (LysM-RLK) CERK1 is a major chitin-binding protein in Arabidopsis thaliana and subject to chitin-induced phosphorylation. J. Biol. Chem. 285, 28902-28911, doi:10.1074/jbc.M110.116657 (2010).

13 Liu, T.T.et al. Chitin-induced dimerization activates a plant immune receptor. Science 336, 1160-1164, doi:10.1126/science.1218867 (2012).

14 Cao, Y. et al. The kinase LYK5 is a major chitin receptor in Arabidopsis and forms a chitininduced complex with related kinase CERK1. Elife, doi:10.7554/eLife.03766 (2014).

15 Willmann, R. et al. Arabidopsis lysin-motif proteins LYM1 LYM3 CERK1 mediate bacterial peptidoglycan sensing and immunity to bacterial infection. Proc. Natl. Acad. Sci. USA 108, 1982419829, doi:10.1073/pnas.1112862108 (2011).

16 Umemoto, N. et al. The structure and function of a soybean beta-glucan-elicitor-binding protein. Proc. Natl. Acad. Sci. USA 94, 1029-1034, doi:DOI 10.1073/pnas.94.3.1029 (1997).

17 op den Camp, R. et al. LysM-type mycorrhizal receptor recruited for rhizobium symbiosis in nonlegume Parasponia. Science 331, 909-912, doi:10.1126/science.1198181 (2011).
18 Buendia, L., Wang, T., Girardin, A. \& Lefebvre, B. The LysM receptor-like kinase SILYK10 regulates the arbuscular mycorrhizal symbiosis in tomato. New Phytol., doi:10.1111/nph.13753 (2015).

19 Miyata, K. et al. The bifunctional plant receptor, OsCERK1, regulates both chitin-triggered immunity and arbuscular mycorrhizal symbiosis in rice. Plant Cell Physiol. 55, 1864-1872, doi:10.1093/ pcp/pcu129 (2014).

20 Zhang, X. W. et al. The receptor kinase CERK1 has dual functions in symbiosis and immunity signalling. Plant J. 81, 258-267, doi:10.1111/ tpj.12723 (2015).

21 Mulder, L., Lefebvre, B., Cullimore, J. \& Imberty, A. LysM domains of Medicago truncatula NFP protein involved in Nod factor perception. Glycosylation state, molecular modeling and docking of chitooligosaccharides and Nod factors. Glycobiol. 16, 801-809, doi:10.1093/glycob/cwl006 (2006).

22 Lefebvre, B. et al. Role of $N$-glycosylation sites and CXC motifs in trafficking of Medicago truncatula Nod factor perception protein to plasma membrane. J. Biol. Chem. 287, 10812-10823, doi:10.1074/jbc.M111.281634 (2012).

23 Ride, J. P. \& Barber, M. S. Purification and characterization of multiple forms of endochitinase from wheat leaves. Plant Sci. 71, 185-197, doi:Doi 10.1016/0168-9452(90)90008-C (1990)

24 El Gueddari, N. E., Rauchhaus, U., Moerschbacher, B. M. \& Deising, H. B. Developmentally regulated conversion of surface-exposed chitin to chitosan in cell walls of plant pathogenic fungi. New Phytol. 156, 103-112, doi:DOI 10.1046/j.14698137.2002.00487.x (2002).

25 Duplessis, S. et al. Obligate biotrophy features unraveled by the genomic analysis of rust fungi. Proc. Natl. Acad. Sci. USA 108, 9166-9171, doi:10.1073/pnas.1019315108 (2011).

26 Veneault-Fourrey, C. et al. Genomic and transcriptomic analysis of Laccaria bicolor CAZome reveals insights into polysaccharides remodelling during symbiosis establishment. Fungal Genet. Biol. 72, 168-181, doi:10.1016/j.fgb.2014.08.007 (2014).

27 Fujikawa, T. et al. Surface a-1,3-glucan facilitates fungal stealth infection by interfering with innate immunity in plants. PLoS Pathog. 8, doi:10.1371/ journal.ppat.1002882 (2012). 
28 Oliveira-Garcia, E. \& Deising, H. B. Attenuation of PAMP-triggered immunity in maize requires down-regulation of the key ß-1,6-glucan synthesis genes KRE5 and KRE6 in biotrophic hyphae of Colletotrichum graminicola. Plant J., doi:10.1111/ tpj.13205 (2016).

29 Bolton, M. D. et al. The novel Cladosporium fulvum lysin motif effector Ecp6 is a virulence factor with orthologues in other fungal species. Mol. Microbiol. 69, 119-136, doi:10.1111/j.1365-2958.2008.06270.x (2008).

30 de Jonge, R. et al. Conserved fungal LysM effector Ecp6 prevents chitin-triggered immunity in plants. Science 329, 953-955, doi:10.1126/science.1190859 (2010).

31 Sánchez-Vallet, A. et al. Fungal effector Ecp6 outcompetes host immune receptor for chitin binding through intrachain LysM dimerization. Elife, doi:10.7554/eLife.00790 (2013).

32 Kombrink, A. \& Thomma, B. P. H. J. LysM Effectors: Secreted proteins supporting fungal life. PLoS Pathog. 9, doi:10.1371/journal.ppat.1003769 (2013).

33 Marshall, R. et al. Analysis of two in planta expressed LysM effector homologs from the fungus Mycosphaerella graminicola reveals novel functional properties and varying contributions to virulence on wheat. Plant Physiol. 156, 756-769, doi:10.1104/pp.111.176347 (2011).

34 Mentlak, T. A. et al. Effector-mediated suppression of chitin-triggered immunity by Magnaporthe oryzae is necessary for rice blast disease. Plant Cell 24, 322-335, doi:10.1105/tpc.111.092957 (2012).

35 Takahara, H. et al. Colletotrichum higginsianum extracellular LysM proteins play dual roles in appressorial function and suppression of chitintriggered plant immunity. New Phytol. (2016).

36 Chen, X. L. et al. $\mathrm{N}$-glycosylation of effector proteins by an alpha-1,3-mannosyltransferase is required for the rice blast fungus to evade host innate immunity. Plant Cell 26, 1360-1376, doi:10.1105/ tpc.114.123588 (2014).

37 Wawra, S. et al. The fungal-specific beta-glucanbinding lectin FGB1 alters cell-wall composition and suppresses glucan-triggered immunity in plants. Nat. Commun. 7, 13188, doi:10.1038/ ncomms13188 (2016).

38 Stergiopoulos, I. et al. Tomato $\mathrm{Cf}$ resistance proteins mediate recognition of cognate homologous effectors from fungi pathogenic on dicots and monocots. Proc. Natl. Acad. Sci. USA 107, 7610-7615, doi:10.1073/pnas.1002910107 (2010).
39 van den Burg, H. A., Harrison, S. J., Joosten, M. H. A. J., Vervoort, J. \& de Wit, P. J. G. M. Cladosporium fulvum Avr4 protects fungal cell walls against hydrolysis by plant chitinases accumulating during infection. Mol. Plant-Microbe Interact. 19, 1420 1430, doi:10.1094/MPMI-19-1420 (2006).

40 Bishop, J. G., Dean, A. M. \& Mitchell-Olds, T. Rapid evolution in plant chitinases: molecular targets of selection in plant-pathogen coevolution. Proc. Natl. Acad. Sci. USA 97, 5322-5327 (2000).

41 Kombrink, A. et al. Verticillium dahliae LysM effectors differentially contribute to virulence on plant hosts. Mol. Plant Pathol., doi:10.1111/mpp.12520 (2016).

42 Naumann, T. A. \& Wicklow, D. T. Chitinase modifying proteins from phylogenetically distinct lineages of Brassica pathogens. Physiol. Mol. Plant Pathol. 82 1-9, doi:10.1016/j.pmpp.2012.12.001 (2013).

43 Karimi Jashni, M. et al. Synergistic action of a metalloprotease and a serine protease from Fusarium oxysporum f. sp. lycopersici cleaves chitinbinding tomato chitinases, reduces their antifungal activity, and enhances fungal virulence. Mol. PlantMicrobe Interact. 28, 996-1008, doi:10.1094/Mpm104-15-0074-R (2015).

44 Rose, J. K. C., Ham, K. S., Darvill, A. G. \& Albersheim, P. Molecular cloning and characterization of glucanase inhibitor proteins: Coevolution of a counterdefense mechanism by plant pathogens. Plant Cell 14, 1329-1345, doi:10.1105/tpc.002253 (2002)

45 Bishop, J. G. et al. Selection on glycine $\beta-1,3-$ endoglucanase genes differentially inhibited by a Phytophthora glucanase inhibitor protein. Genet. 169, 1009-1019, doi:10.1053/genetics.103.025098 (2005).

46 Sánchez-Rangel, D., Sánchez-Nieto, S. \& Plasencia, J. Fumonisin B1, a toxin produced by Fusarium verticillioides, modulates maize $\beta$-1,3-glucanase activities involved in defense response. Planta 235 , 965-978, doi:10.1007/s00425-011-1555-0 (2012).

47 Brown, G. D. Innate antifungal immunity: The key role of phagocytes. Annu. Rev. Immunol. 29, 1-21, doi:10.1146/annurev-immunol-030409-101229 (2011).

48 Romani, L. Immunity to fungal infections. Nat. Rev. Immunol.11, 275-288, doi:10.1038/nri2939 (2011).

49 Rappleye, C. A., Eissenberg, L. G. \& Goldman, W. E. Histoplasma capsulatum a-(1,3)-glucan blocks innate immune recognition by the $\beta$-glucan receptor. Proc. Natl. Acad. Sci. USA 104, 1366-1370, doi:10.1073/pnas.0609848104 (2007) 
50 Beauvais, A. et al. Deletion of the $a-(1,3)$-glucan synthase genes induces a restructuring of the conidial cell wall responsible for the avirulence of Aspergillus fumigatus. PLoS Pathog. 9, doi:10.1371/ journal.ppat.1003716 (2013).

51 Aimanianda, V. et al. Surface hydrophobin prevents immune recognition of airborne fungal spores. Nature 460, 1117-U1179, doi:10.1038/ nature08264 (2009).

52 Bueter, C. L., Specht, C. A. \& Levitz, S. M. Innate sensing of chitin and chitosan. PLoS Pathog. 9, doi:10.1371/journal.ppat.1003080 (2013).

53 Battaglia, E. et al. Carbohydrate-active enzymes from the zygomycete fungus Rhizopus oryzae: a highly specialized approach to carbohydrate degradation depicted at genome level. BMC Genom. 12, 1-12, doi:10.1186/1471-2164-12-38 (2011).
54 Lowe, R. G. T. \& Howlett, B. J. Indifferent, affectionate, or deceitful: Lifestyles and secretomes of fungi. PLoS Pathog. 8, doi:10.1371/ journal.ppat.1002515 (2012).

55 de Jonge, R. \& Thomma, B. P. H. J. Fungal LysM effectors: extinguishers of host immunity? Trends Microbiol. 17, 151-157, doi:10.1016/j. tim.2009.01.002 (2009). 



\section{Summary}

Microorganisms establish symbiotic relationships with plants that range from mutualistic to pathogenic. During plant colonization, microbes secrete effector proteins that manipulate host physiology to their advantage. In turn, host plants employ receptors that recognize microbe-derived or modified-self molecules indicative of invasion. Over the past 70 years, our view on the concepts that describe the mechanisms underlying plant-microbe interactions has drastically changed (Chapter 1). In order to enhance our understanding on the molecular interplay between host and microbe, the work presented in this thesis was designed to further unravel components involved in the recognition of the two fungal pathogens Verticillium dahliae and Cladosporium fulvum, as well as the functions of effector proteins produced by these pathogens during tomato infection.

Chapter $\mathbf{2}$ provides an overview of the various functions displayed by effector molecules of phytopathogenic filamentous microbes, specifically their roles in the suppression of plant immune responses. However, effectors are not unique to pathogens but are employed by any microbe that encounters immune responses during plant host colonization. Moreover, plant-microbe interactions occur in environments that contain additional microbial partners, which can affect the colonizing microbe as well as the host plant. Thus, we propose that effector molecules are involved in microbial competition or cooperation in addition to their role in host manipulation, and may, therefore, shape the plant microbiome.

The carbohydrate-binding lysin motif (LysM) occurs in all living organisms, except Archaea. In fungal pathogens, effectors with varying numbers of LysMs have been implicated in the suppression of chitin-triggered immunity. The LysM effector Ecp6, which is secreted by the tomato pathogen C. fulvum during infection, contains three LysM domains and is able to bind chitin with high affinity. Specifically, Ecp6 disturbs chitin-induced immune responses by binding chitin with ultrahigh affinity through intramolecular LysM dimerization, resulting in its capacity to outcompete plant chitin receptors. Additionally, the singular LysM domain (LysM2), which is not involved in intramolecular chitin binding, can perturb chitin responses in tomato through a yet unknown mechanism. Due to its relatively low affinity for chitin, it has been hypothesized that LysM2 interferes with chitin receptor dimerization required for the activation of immunity. In Chapter 3 we further investigate Ecp6 functionality in the model plant Arabidopsis thaliana, for which chitin perception has been intensely studied. We show that Ecp6 suppresses chitin-mediated immune responses in $A$. thaliana and affects internalization of the LysM receptor kinase AtLYK5 in a ligand-dependent manner.

All plant chitin receptors identified to date belong to the class of surface-localized LysMcontaining receptor proteins. Chapter 4 describes a chitin affinity-purification approach followed by mass spectrometry aimed at the identification of LysM receptor molecules in tomato. We identified two chitin-binding LysM receptor molecules that are closely related to $A$. thaliana LYK4 and rice CEBiP, respectively, both of which have been implicated in chitin 
recognition in those plant species. While silencing of SILYK4 resulted in reduced tomato responsiveness to chitin, silencing of SICEBiP had little to no effect. Since SILYK4 carries an inactive intracellular kinase domain we hypothesize that it is involved in the formation a of heteromeric chitin receptor complex, similar to $A$. thaliana and rice. Our findings suggest that SILYK4 is a major component of the chitin receptor complex in tomato that activates the canonical chitin signal transduction pathway.

In contrast to C. fulvum, $V$. dahliae infects over 200 different plant species. Like other pathogens, $V$. dahliae utilizes effector proteins to manipulate its hosts. We previously identified the effector protein Ave1, which greatly contributes to virulence during plant colonization, through comparative genomics. Ave1 homologs are ubiquitous in plants and occur in several other plant pathogens. As the distribution of the microbial homologs does not follow the phylogeny of the species they occur in, it has been hypothesized that $V$. dahliae acquired Ave1 via horizontal gene transfer. Interestingly, all homologs carry a conserved plant natriuretic peptide (PNP) sequence and $V$. dahliae Ave1 displays PNP activity (Chapter 5). However, complementation of a V. dahliae Ave 1 deletion mutant with microbial and plant homologs that also display PNP activity does not reinstall virulence. Thus, the contribution of $V$. dahliae Ave 1 to virulence does not depend on its PNP activity. Instead, in contrast to its homologs, Ave1 additionally interacts with, and inhibits, plant endochitinases to interfere with host immunity. These findings demonstrate that Ave1 has functionally diverged from its homologs following horizontal gene transfer and evolved a novel function in plant immune suppression.

In tomato, $V$. dahliae Ave1 is recognized by the extracellular leucine-rich repeat-containing receptor protein (eLRR-RP) Ve1 resulting in resistance to $V$. dahliae. However, no function could be ascribed to the closest homolog of the Ve1 receptor, Ve2. Previous analyses of domain swap mutants between Ve1 and Ve2 have highlighted the role of the eLRR region in Ave1 ligand perception. In Chapter 6, we investigated the physical interaction between Ve1 and its ligand using a biochemical approach. As has been suggested previously based on the receptor mutant analyses, not only Ve1 but also Ve2 is able to bind Ave1. This binding is not solely mediated by a single region in the eLRR domain but rather requires multiple clusters of scattered eLRRs. Moreover, we show that Ave1 binding is required for the recruitment of the regulatory receptor kinase BAK1 to the Ve1 receptor complex, which represents a hallmark of eLRR-RP immune signaling.

In addition to chitin, B-glucans are major cell wall components of filamentous microbes that elicit plant immune responses. The widespread capacity of glycan perception in plants has driven the evolution of various strategies that help filamentous microbes to evade detection. Chapter $\mathbf{7}$ synthesizes the findings presented in this thesis and places them into the broader perspective of glycan-triggered plant immunity and the strategies that evolved in plant-associated microbes to suppress it. 


\section{Acknowledgements}

I have always dreaded the moment of writing "the acknowledgements". Thinking about it now, I am not sure whether this was due to the worry of not knowing what to write or the realization that all good things eventually end. As absurd as this thought might have seemed during the $\mathrm{PhD}$, having reached the end I can now safely say that I have enjoyed each step of this interesting journey, which has allowed me to learn and grow both professionally and personally. Of course, none of this would have been possible without the support of my promotors Bart and Pierre. Ever since I joined Pierre's group for my M.Sc. thesis I have been inspired by his enthusiasm and dedication to science. Pierre, you are a great mentor and I am grateful for your constructive criticism throughout the last years. Without your encouragement this path would undoubtedly have been much rougher. Bart, I consider myself very lucky that you gave me the opportunity to join your group as PhD candidate. Even though it took me a while to get used to it, the professional freedom and independence you gave me was invaluable. I was often surprised to realize how well you understood my need for reassurance, and your optimism and trust helped me gain confidence both in my work and myself. Thank you for that.

For anyone who happens to flick through the pages of this book, I think it becomes evident that the presented work is the result of a concerted effort. Over the years, there were many people who provided practical support and advice, some of which I would particularly like to thank here: Yin, for providing lots and lots of plasmids and bacterial strains whenever I asked; Dirk-Jan, for introducing me to the "art of running the bioreactor"; Malaika and Hui, for their help at any time, for example with punching leaf discs and processing samples especially during the last year; Grardy for solving cloning issues, cakes, and early morning chats; and Michael, Xiaoqian and Luigi for valuable insights into bioinformatic analyses. I was also lucky enough to supervise several excellent students who have greatly contributed to this thesis. Nick, I think it goes without mentioning that your work on the Ave1 purification was crucial for the progress of the project. I am very glad that you agreed to pick up where I left off during your $\mathrm{PhD}$, and am sure that the project is in very capable hands. Wendy and Hamid, without your help with the generation of the many Verticillium complementation mutants, tomato inoculations and rounds of symptom assessment I would not have been able to complete the Ave1 chapter. I would also like to thank Shiva, Giulio and Yiru who have worked on projects that are not presented here. Your contributions to those projects were greatly appreciated and will hopefully be followed up soon.

In addition to the people who have directly contributed to experiments, I would like to express my gratitude to all those who have provided ideas and criticism during many indispensable discussions. I would especially like to mention Mireille, Luigi, David, Andrea, Scott, Anja, Daniela and Carl who never grew tired of my questions and have helped me 
many times to objectively assess my work instead of drowning in worries. Your advice and insight have been and will always be very much appreciated.

I am very glad that I had the privilege to belong to the Verticillium group and the laboratory of Phytopathology. It was more a rule than an exception that we received scolding looks or complaints from passers-by during our frequent and lively coffee breaks with cake ("Are they having cake again?", we would hear the people from neighboring departments ask). This is to say that I have genuinely enjoyed working in this department, with its very warm, friendly and welcoming atmosphere. The many social activities and chitchats always inspired and motivated me. Surely, the department would not be the same without the effort and dedication of our secretary Ali and the technical staff (Ester, Grardy, Laurens, Natalie, and Rob). Thank you for keeping the lab well organized and for making sure that we're never short of anything.

Many thanks also to all fellow PhDs at Phytopathology - it was great fun to work with you. I very much appreciated the interesting discussions during our regular $\mathrm{PhD}$ meetings and occasional trial defenses. Johan, Chara and Elysa, thank you for the many chats, your help with troubleshooting and for your friendship.

Jordi, more than anything I would like to thank you for your lab companionship, which I greatly missed during the last year of my PhD. For a long time we worked on different aspects of the same project, and the fact that I could share both failures and successes with you was of invaluable importance to me. Despite our different working strategies we have managed to put together an excellent review, which I consider a real accomplishment. For these and many other reasons, I wanted to have you next to me as one of my paranymphs on this important day.

Setareh, my other paranymph, in you I have found an inspiring, honest, reliable and compassionate colleague and friend. I admire your pragmatic attitude and value your critical opinion. I sincerely hope that we will keep on discussing and laughing together for many years to come.

To the rest of the EPS ladies - Lot and Lotte, we met in the formal setting of the EPS PhD council but you, together with Magda and Setareh, have since become my reference point for professional and personal issues. I hope we can soon get together for another evening of "Friday drinks"; it is overdue!

Parts of the work presented here were carried out in the Department of Plant Cell Biology in Göttingen in the group of Volker Lipka. Volker, thank you for hosting me and for providing valuable input to the Ecp6 project. I would also like to particularly thank Elena, who introduced me to the many aspects of chitin-triggered immunity from the plants' point of view. I am very satisfied with the outcome of my stay and am sure that our continued collaboration will result in at least two very nice publications. 
Life in Wageningen would not have been the same without the many lovely people I met during the past seven years and who have become very dear friends: Céline, Pavlos, Luigi, Alice, Jordi, Iliana, Amalita, Scott, Andrea, Daniela, Anja, Xiaoqian, Tim, Jule, Alexander, Giulia, Christos, Valentina, Rafa, João, Mira, Roxana, Christoph, Andres, Sanne, Sebas, Anna, Mario, Sara, Nelson, and Kim. It is difficult to put into words how much I value the friendship with each and every one of you. However, I am going to say this: You make my life so much richer! I am grateful for all the time spent together and am looking forward to the time ahead.

At this point, I would also like to thank the two people who have made the very last stretch of preparing the final version of this book very, very easy for me. First of all, thanks to my cousin Anja for investing much time and effort in designing the beautiful cover of this thesis. I have already selected two of your prints to be framed. And then, of course, Iliana - I hope you like the present you made me! I cannot thank you enough for your efficiency, professional work, advice and for your patience.

To my family - Vielen Dank an alle Omas und Opas, Tanten, Onkel, Cousinen und Cousins von denen ich mich, trotz der geographischen Entfernung immer unterstützt gefühlt habe. Mir ist bewusst, dass es nicht immer einfach ist zu verstehen, was jemanden dazu motivieren könnte in der Wissenschaft zu arbeiten. Ich bin euch sehr dankbar dafür, dass ihr trotzdem immer Interesse und Verständnis aufbringen konntet.

Mama und Papa, vielen Dank dafür, dass ihr mich auf diesen langen Weg geschickt habt. Ohne eure Ermunterung hätte ich die Strapazen der vielen und häufigen Veränderungen nicht auf mich nehmen können und wollen. Ich bin stolz und froh über euer Vertrauen, dass ich garantiert meine selbstgesteckten Ziele erreichen würde.

Eva, liebe Schwester, wir sind uns garantiert nicht immer eins. Trotzdem bin ich sehr glücklich darüber, dass wir uns gegenseitiges Vertrauen schenken und zusammen halten, wenn es drauf ankommt. Ich danke dir für deinen Optimismus und vor allem dafür, dass du mir immer wieder hilfst meine Sorgen zu relativieren.

Ale, mi vida, hay tantas cosas por las cuales te tengo que agradecer. Aquí, más que nada, gracias por tu infinita paciencia, tu capacidad de animarme y calmarme cuando más lo necesite. Sin vos a mi lado no habría llegado a este punto. Te amo! 



\section{About the author}

Hanna J. Rövenich was born in Berlin, Germany, on January $22^{\text {nd }} 1988$. In 2007, she began her B.Sc. studies in Biotechnology (International First Level Degree "Job Creation Oriented Biotechnology") as part of the Lifelong Learning Programme of the European Union at the University of Perugia, Italy. During the first year of her undergraduate studies, Hanna performed an internship in the Infection and Cancer Biology group of Dr. Massimo Tommasino under the daily supervision of Dr. Rosita Accardi at the International Agency for Research on Cancer in Lyon, France, where she studied the role of the transcription factor isoform $\Delta N p 73$ in EpsteinBarr virus-infected cells. In 2009, she joined the group of Dr. Sally Power for an additional internship at Imperial College London, United Kingdom, to investigate the combined impact of nitrogen and ozone on Trifolium repens and Festuca ovina as representatives of natural plant communities. To complete her B.Sc., Hanna worked on a six-months thesis project entitled "Cytokinin and dormancy in Arabidopsis thaliana" under the supervision of Dr. David Hanke at the University of Cambridge, United Kingdom.

In order to combine her interests in immunology and plant biology, she started her M.Sc. studies in Plant Biotechnology at Wageningen University with the specialization in Molecular Plant Breeding and Pathology. In 2011, she joined the group of Prof. Dr. Pierre de Wit for her M.Sc. thesis project. Together with Dr. Bilal Ökmen, she used a proteomic approach to identify novel effector proteins of the tomato leaf mold pathogen Cladosporium fulvum. Her thesis was awarded the WUF-KLV award for the best M.Sc. thesis of the academic year 2011/2012. Hanna then visited the group of Prof. Dr. Cyril Zipfel at The Sainsbury Laboratory. Under the supervision of Dr. Jacqueline Monaghan, she worked on a sensitized forward-genetic screen of A. thaliana mutants in the immunodeficient background bak1-5 that led to the identification of the calcium-dependent protein kinase CPK28 and the subtilisin-like serine protease S1P as negative regulators of plant immunity upon pathogen perception mediated by surfacelocalized receptor kinases. In 2012, she was awarded her M.Sc. degree with distinction.

Later that year, Hanna joined the Verticillium group at the Laboratory of Phytopathology, Wageningen University, under the supervision of Prof. Dr. Bart Thomma as PhD candidate. Her research on the molecular components involved in the recognition of fungal pathogens and the strategies they evolved to evade host immunity resulted in the publication of this thesis. In 2015, she carried out a three-months stay in the group of Prof. Dr. Volker Lipka at the Georg-August-University in Göttingen, Germany, within the framework of the SUSTAIN COST Action FA1208. During her PhD, Hanna was an active member of the Wageningen PhD Council (WPC) and the PhD Council of the graduate school of Experimental Plant Sciences (EPS), which she chaired in 2014/2015. As of February 2017, Hanna is a postdoctoral researcher in the group of Prof. Dr. Alga Zuccaro at the University of Cologne, Germany, where she investigates the mechanisms underlying $\beta$-glucan recognition in plants and its importance in the establishment of mutualistic relationships between plants and fungi. 


\section{List of publications}

Rovenich H., Snelders N.C.*, Boshoven J.C.*, Grandellis C., Seidl M.F., Boeren S., Ogata M., Ottado J., Mesters J.R., Thomma B.P.H.J. (2017) Neofunctionalisation upon horizontal gene transfer: birth of a fungal effector that evolved a function in immune suppression. In preparation.

*equal contribution

Rovenich H., Petutschnig E.K., Valerius O., Ebert E.K., van den Berg G.C.M., Thomma B.P.H.J. (2017) A LysM receptor kinase mediates chitin-triggered defence responses in tomato. In preparation.

Rovenich H., van der Burgh A., van Damme M., Snelders N.C., Thomma B.P.H.J. (2017) Inactive tomato immune receptor homolog fails to recruit signaling components upon ligand perception. In preparation.

Cook D.E. ${ }^{\dagger}$, Espejo Valle-Inclan J. ${ }^{\dagger}$, Rovenich H., Thomma B.P.H.J.*, Faino L.* (2017) Long Read Annotation (LoReAn): automated eukaryotic genome annotation pipeline incorporating long-read cDNA sequencing. In preparation.

${ }^{t, *}$ equal contribution

Mesarich C.H., Ökmen B., Rovenich H., Griffiths S.A., Wang C., Karimi Jashni M., Mihajlovski A., Collemare J., Hunziker L., Deng C.H., van der Burgt A., Beenen H.G., Templeton M.D., Bradshaw R.E., de Wit P.J.G.M. (2017) Specific hypersensitive response-associated recognition of new apoplastic effectors from Cladosporium fulvum in wild tomato. Submitted.

Boshoven J.C., Ebert M.K.*, Song Y.*, Rovenich H., Rojas Padilla E., Bolton M.D., Thomma B.P.H.J. (2017) Homologs of Verticillium dahliae effector Ave1 contribute to virulence of various fungal pathogens. Submitted.

*equal contribution

Song Y.*, Zhang Z.*, Boshoven J.C., Rovenich H., Seidl M.F., Jakse J., Maruthachalam K., Liu C.-M., Subbarao K., Javornik B., Thomma B.P.H.J. (2017) Tomato immune receptor Ve1 recognizes surface-exposed co-localized $\mathrm{N}$ - and $\mathrm{C}$-termini of Verticillium dahliae effector Ave1. bioRxiv 103473; DOI: doi.org/10.1101/103473.

*equal contribution

Stegmann M., Monaghan J., Smakowska E., Rovenich H., Lehner A., Holton N., Belkhadir Y., Zipfel C. (2017) The receptor kinase FER is a RALF-regulated scaffold controlling plant immune signaling. Science, DOI: 10.1126/science.aal2541. 
Kombrink A., Rovenich H.*, Shi-Kunne X.*, Rojas Padilla E.*, van den Berg G.C.M.*, Domazakis E., de Jonge R., Valkenburg D.J., Sánchez-Vallet A., Seidl M.F., Thomma B.P.H.J. (2016) Verticillium dahliae LysM effectors differentially contribute to virulence on plants. Molecular Plant Pathology, DOI: 10.1111/mpp.12520.

*equal contribution

Rovenich H., Zuccaro A., Thomma B.P.H.J. (2016) Convergent evolution of fungal microbes towards the evasion of glycan-triggered immunity. New Phytologist, DOI: 10.1111/ nph.14064.

Monaghan J., Matschi S., Shorinola O., Rovenich H., Matei A., Segonzac C., Gro-Malinovsky F., Rathjen J.P., MacLean D., Romeis T., Zipfel C. (2014) The calcium-dependent protein kinase CPK28 buffers plant immunity and regulates BIK1 turnover. Cell Host Microbe, DOI: 10.1016/j.chom.2014.10.007.

Rovenich H.* ${ }^{*}$ Boshoven J.C.*, Thomma B.P.H.J. (2014) Filamentous pathogen effector functions: Of pathogens, hosts and microbiomes. Current Opinion Plant Biology, DOI:10.1016/j.pbi.2014.05.001.

* equal contribution

Fradin E.F.*, Zhang Z.*, Rovenich H. ${ }^{\S}$, Song Y. ${ }^{\S}$, Liebrand T.W.H,. Masini L., van den Berg G.C.M., Joosten M.H.A.J., Thomma B.P.H.J. (2014) Functional analysis of the tomato immune receptor Ve1 through domain swaps with its non-functional homolog Ve2. PLOS ONE, DOI:10.1371/journal.pone.0088208.

*, equal contribution 



\title{
Education Statement of the Graduate School Experimental Plant Sciences
}

\author{
Issued to: Hanna Rövenich \\ Date: 29 August 2017 \\ Group: Phytopathology \\ University: Wageningen University \& Research
}

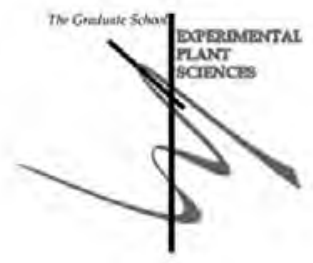

\section{1) Start-up phase}

date

- First presentation of your project

Dissection of Ve1-mediated immunity

12 Nov 2012

- Writing or rewriting a project proposal

Dissection of Ve1-mediated immunity

Oct 2012

- Writing a review or book chapter

Filamentous pathogen effector functions: Of Pathogens, Hosts and Microbiomes,

May 2014

Current Opinion in Plant Biology 2014, 20:96-103. doi:10.1016/j.pbi.2014.05.001

- MSc courses

Laboratory use of isotopes

\section{Subtotal Start-up Phase}

13.5 credits $^{*}$

2) Scientific Exposure

date

- EPS PhD student days

EPS PhD student day, Amsterdam, NL

30 Nov 2012

EPS PhD student day, Leiden, NL

29 Nov 2013

EPS PhD student retreat "Get2Gether", Soest, NL

29-30 Jan 2015

- EPS theme symposia

EPS Theme 2: Interactions between Plants and Biotic Agents, Utrecht, NL

24 Jan 2013

EPS Theme 2: Interactions between Plants and Biotic Agents, Amsterdam, NL

25 Feb 2014

EPS Theme 2: Interactions between Plants and Biotic Agents, Utrecht, NL

20 Feb 2015

EPS Theme 2: Interactions between Plants and Biotic Agents, Leiden, NL

22 Jan 2016

- Annual meetings (national) and other national platforms

Annual meeting 'Experimental Plant Sciences', Lunteren, NL

22 Apr 2013

Annual meeting 'Experimental Plant Sciences', Lunteren, NL

14-15 Apr 2014

Annual meeting 'Experimental Plant Sciences', Lunteren, NL

13-14 Apr 2015

- Seminars (series), workshops and symposia

Workshops

COST SUSTAIN workshop "Structure-guided investiagtion of effector function, action and recognition", Bucharest, Romania

Workshop BU Biointeractions \& Plant Health - WU Phytopathology, Wageningen, NL

Symposia

Plantum "Intraspecific Pathogen Variation - Implications and Opportunities",

Wageningen, NL

Mini-Symposium "How to write a world-class paper", Wageningen, NL

Farewell symposium Pierre de Wit, Wageningen, NL

Mini-Symposium Phytopathology, Wageningen, NL

Mini-Symposium "From gene to protein and beyond", Annual Internal CLIB-GC

Retreat, Bonn, Germany

SFB1101 Symposium "Molecular encoding of plant processes", Tuebingen, Germany

"Rewriting our genes" with Jennifer Doudna and Edze Westra, Wageningen, NL

10-12 Sep 2014

10 Feb 2015

$22 \operatorname{Jan} 2013$

17 Oct 2013

06 May 2014

24 Nov 2014

22 Oct 2015

04-06 Apr 2016 30 Sep 2016 
Seminars

Writing for high-impact journals (Andrew Sugden, Editor of Science)

08 Feb 2013

A. thaliana as a model system for the study of evolutionary questions (Detlef Weigel), EPS flying seminar

Molecular insights into spore biology and metabolism of P. infestans, the potato late blight pathogen (Howard S. Judelson), EPS flying seminar

Integrative genomics of desctructive pathogens from oomycetes to malaria parasites (Rays Jiang), EPS flying seminar

Invited seminar Brian Staskawicz

A "GoogleMAP"-type molecular view of microbes - from culture to people (Peter Dorrestein)

Soilborne pathogens and their natural biocontrol agents in cereal-based production systems (David Weller)

Endophytes in agriculture - evaluating their application via metabolomics and genomics (Ross Mann)

Back to the roots (Jos Raaijmakers)

Plant Sciences Seminar: Mutualism in Action (Toby Kiers and René Geurts)

From protein solution to single crystal $\mathrm{X}$-ray diffraction: Chitin binding by LysM

domains at atomic resolution (Jeroen Mesters)

Reprogramming cells for defence in plant innate immunity (Jane Parker)

Plant Metacaspases (Frank van Breusegem)

Dissecting the interactions between Phytophthora sojae and soy bean: making sense of signaling and effectors (Yuanchao Wang)

Evolution of plant-herbivore interactions: insights from genomics (Noah Whiteman)

Chromatin structure controls centromeres and secondary metabolism in filamentous fungi (Michael Freitag)

Genetics and epigenetics: a complex relationship (Ortrun Mittelstein-Scheid)

The evolutionary significance of gene and genome duplications (Yves van den Peer)

How Ralstonia solanacearum succeeds in plant xylem vessels (Caitilyn Allen)

Effectors as molecular probes to understand pathogenesis (Wenbo Ma)

$27 \mathrm{Feb} 2013$

07 May 2013

07 May 2013

21 May 2013

23 Aug 2013

25 Sep 2013

04 Oct 2013

07 Jan 2014

11 Mar 2014

31 Mar 2014

09 Apr 2014

09 Apr 2014

16 Jul 2014

17 Jul 2014

21 Oct 2014

19 Nov 2014

03 Feb 2015

29 Apr 2016

20 Jun 2016

\section{- Seminar plus}

Michael Freitag, Oregon State University, USA

21 Oct 2014

Caitilyn Allen, University of Wisconsin-Madison, USA

29 Apr 2016

- International symposia and congresses

Keystone Symposium: Plant Immunity - Pathways and Translation, Big Sky, MT, USA

07-12 Apr 2013

5th $\mathrm{PhD}$ retreat (EPSR), Ghent, Belgium

23-26 Jul 2013

28th Fungal Genetics Conference, Pacific Grove, CA, USA

17-22 Mar 2015

12th New Phytologist workshop "The apoplast as battleground for plant-microbe

09-10 Jul 2015

interactions", Rauischholzhausen, Germany

03 Jun 2014

Functional analysis of the fungal effector Ave1, Spring school 'Host-microbe

10 Sep 2014

Molecular mechanisms underlying the Verticillium-tomato interaction, COST SUSTAIN

workshop, Bucharest, Romania

Work on effectors and receptors in Phytopathology, Workshop BU Biointeractions \&

10 Feb 2015

Plant Health - WU Phytopathology, Wageningen, NL

The Verticillium effector Ave1 contributes to fungal virulence through inhibition of

plant chitinases, ALW meeting 'Experimental Plant Sciences', Lunteren, NL 
Fungal strategies to subvert host chitin-triggered immunity, Seminar CEPLAS (Groups

18 Sep 2015

Doehlemann and Zuccaro), Institute for Genetics, University of Cologne, Cologne,

Germany

Fungal strategies to subvert host chitin-triggered immunity, Mini-Symposium,

22 Oct 2015

Annual Internal CLIB-GC Retreat, Bonn, Germany

Posters

The tomato receptor-like protein Ve1 interacts with the fungal effector Ave1 to

10 Apr 2013

initiate immune signaling, Keystone Symposium, MT, USA

The fungal effector Ave1 is a putative inhibitor of plant chitinases, COST SUSTAIN

10 Sep 2014

workshop, Bucharest

The Verticillium effector Ave1 contributes to fungal virulence through inhibition of

20 Mar 2015

plant chitinases, 28th Fungal Genetics Conference, Pacific Grove, CA, USA

The Verticillium effector VdAve1 is a dual function protein, SFB1101 symposium,

Tuebingen, Germany

04 Apr 2016

$\checkmark$ IAB interview

- Excursions

\section{3) In-Depth Studies}

Subtotal Scientific Exposure

24.9 credits* $^{*}$

- EPS courses or other PhD courses

PhD course: Introduction Bioinformatics 'A User's Approach', Wageningen, NL

date

PhD course: Advanced proteomics, Wageningen, NL

27-31 Aug 2012

PhD spring school 'Host-microbe interactomics', Wageningen, NL

23-26 Apr 2013

Journal club

02-04 Jun 2014

Member of the Verticillium literature discussion group of Phytopathology

$2012-2016$

- Individual research training

Short term scientific mission (COST FA1208 SUSTAIN Action) at the Schwann-

Schleiden Research Centre, Department of Plant Cell Biology, Laboratory of Prof.

07 Sep-30 Nov

Volker Lipka, Göttingen, Germany

\begin{tabular}{|c|c|c|c|}
\hline & & Subtotal In-Depth Studies & 9.6 credits* $^{*}$ \\
\hline \multicolumn{2}{|c|}{ 4) Personal development } & & $\underline{\text { date }}$ \\
\hline \multirow[t]{2}{*}{$\triangleright$} & Skill training courses & & \\
\hline & PhD Competence Assessment & & May-Jun 2013 \\
\hline \multirow[t]{3}{*}{ 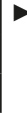 } & Organisation of PhD students day, course or conference & & \\
\hline & ExPectations Day “Communication and ethics in science” & & 28 Mar 2014 \\
\hline & Get2Gether PhD Student Retreat, Soest, NL & & 29-30 Jan 2015 \\
\hline \multirow[t]{3}{*}{ 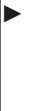 } & Membership of Board, Committee or PhD council & & \\
\hline & Member EPS PhD council & & Mar 2013-Apr 2014 \\
\hline & Chair of the EPS PhD council & & Apr 2014-Apr 2015 \\
\hline
\end{tabular}

Subtotal Personal Development

5.4 credits*

Herewith the Graduate School declares that the PhD candidate has complied with the educational requirements set by the Educational Committee of EPS which comprises of a minimum total of 30 ECTS credits

${ }^{*}$ A credit represents a normative study load of 28 hours of study. 
This work was supported by a VICl grant from the Research Council for Earth and Life sciences (ALW) of the Netherlands Organization for Scientific Research (NWO). The work was carried out in the Laboratory of Phytopathology, Wageningen University, the Netherlands.

Cover design: Anja Lomparski

Cover layout: lliana Boshoven-Gkini | AgileColor.com

Thesis layout: lliana Boshoven-Gkini | AgileColor.com

Print: GVO drukkers \& vormgevers | gvo.nl 


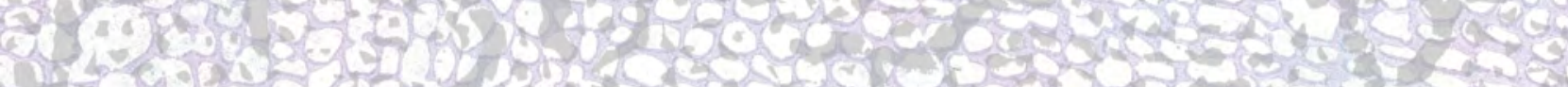
1.

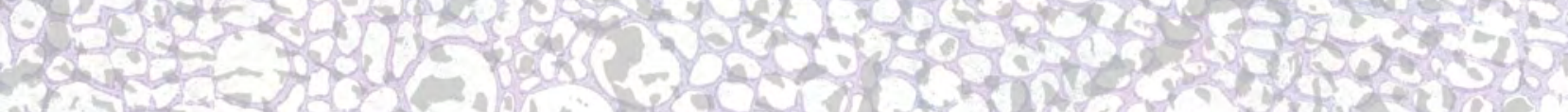

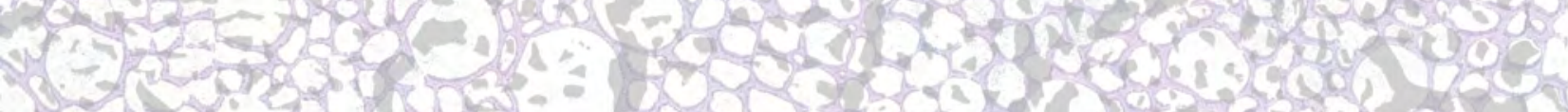

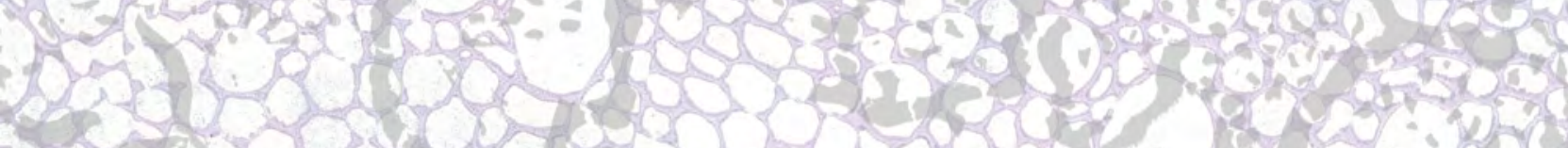

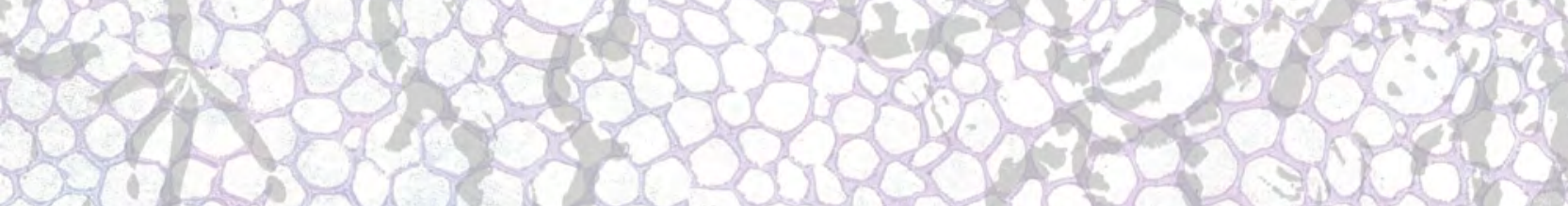

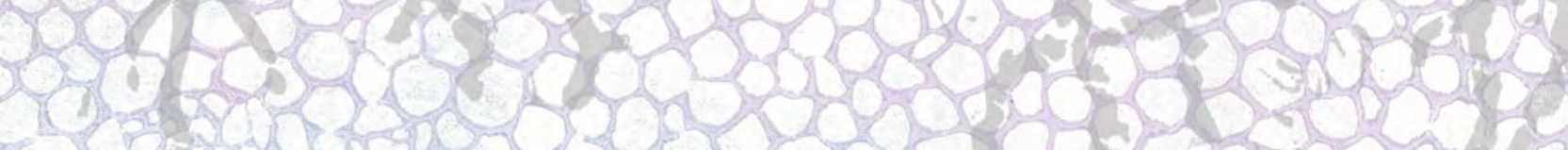
S.9.

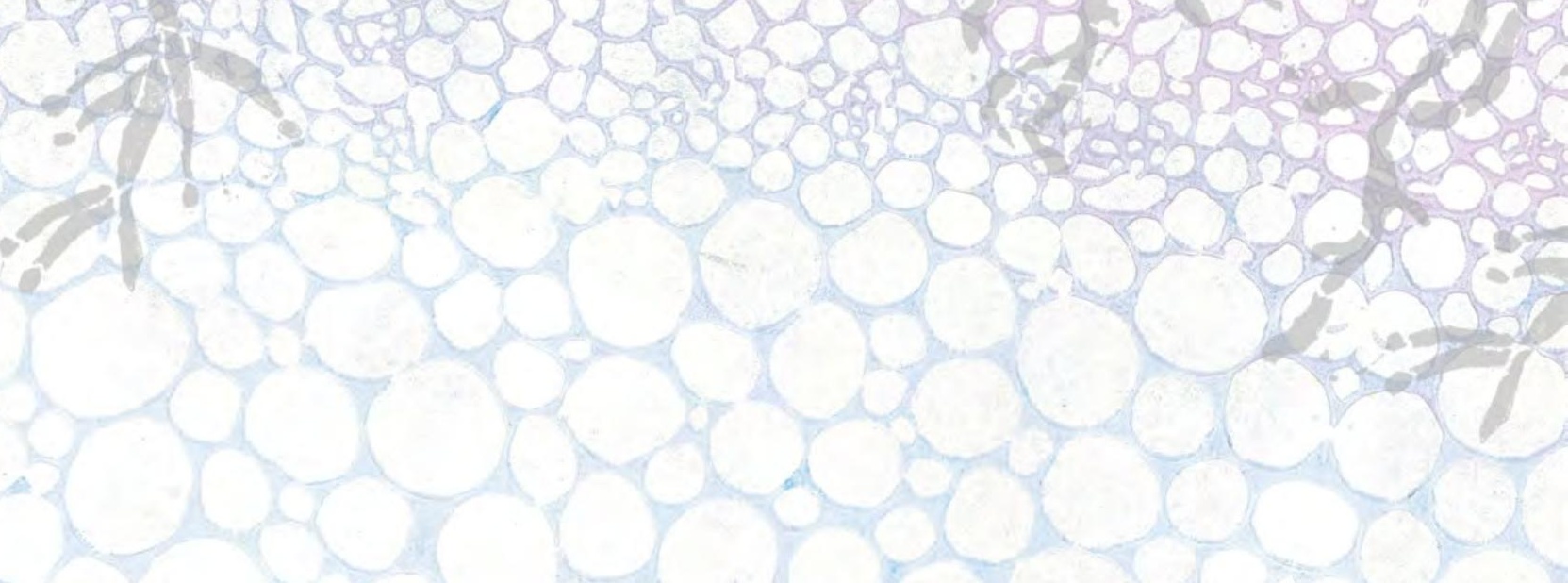

